\title{
34TH ANNUAL SCIENTIFIC MEETING OF THE CANADIAN PAIN SOCIETY: ABSTRACTS
}

WEDNESDAY MAY 8, 2013

1

KEYNOTE SPEAKER - 9:00 AM - 9:45 AM

MARY ELLEN JEANS LECTURE

Chair: Jeffrey $S$ Mogil

EP Taylor Professor of Pain Research, Canada Research Chair in the Genetics of Pain (Tier I), Department of Psychology and Alan Edwards Centre for Pain Research, McGill University, Montreal, Quebec; 2013 Scientific Program Co-Chair, Canadian Pain Society

\section{$1 \mathrm{~A}$}

\section{FROM MECHANISMS TO MEDICINES: THERE'S MANY A} SLIP 'TWIXT CUP AND LIP

Invited Speaker: Robert H Dworkin

Professor of Anesthesiology, Neurology, Oncology, and Psychiatry;

Professor of Neurology in the Center for Human Experimental

Therapeutics; Director, Analgesic, Anesthetic, and Addiction

Clinical Trial Translations, Innovations, Opportunities, and

Networks (ACTTION), a public-private partnership with the FDA,

University of Rochester School of Medicine and Dentistry,

Rochester, New York

Learning Objectives:

1. To review explanations for false positive and negative results in chronic pain clinical trials.

2. To describe research efforts and various initiatives designed to decrease false positive and negative results.

3. To discuss the implications of false positive and negative results for developing chronic pain treatment recommendations.

A substantial percentage of patients with chronic pain are refractory to existing pharmacologic treatments, and the patients who do respond to these treatments typically obtain only partial relief of their pain. Considerable effort is therefore being devoted to the development of new treatments for chronic pain that provide either meaningful benefits to larger percentages of patients or greater pain relief for those who do respond to treatment. This presentation will discuss research efforts and various initiatives designed to decrease false positive and false negative results and thereby potentially accelerate the identification of analgesic medications with improved efficacy and safety. The challenging implications of the results in recent clinical trials for developing treatment recommendations for patients with chronic pain will also be briefly discussed.

\section{SESSION $101-10: 30$ AM}

2

\section{ETHICAL CONTROVERSIES IN PAIN RESEARCH}

Chair: Christine T Chambers

Speakers: Christine T Chambers; Joel Katz; Jeffrey S Mogil

WORKSHOP OBJECTIVE:

The scientific study of pain often involves the application of painful stimuli and/or the withholding of pain relief in research subjects. Guidelines exist for the ethical conduct of pain research with humans and animals, yet challenges still arise. This symposium will describe ethical issues encountered by pain researchers, with examples from pediatric, adult, and animal studies.

Learning Objectives:

1. To identify ethical issues commonly encountered in pain research.

2. To consider the necessity of experimentally induced pain on children and animals

3. To understand the ethics of deception in human experimental pain research

\section{A}

USE OF EXPERIMENTALLY-INDUCED PAIN IN CHILDREN: ETHICAL ISSUES AND OPPORTUNITIES

Christine T Chambers

Dalhousie University \& IWK Health Centre, Halifax, Nova Scotia

The cold pressor task (CPT) is a lab-based method of inducing pain that requires an individual to submerse his/her hand or forearm in cold water for as long as can be tolerated (up to several minutes). Ethical concerns have been raised regarding the non-therapeutic induction of pain in children, who may not have the capacity to provide fully informed consent for participation. We will provide an overview of the ethical challenges we have encountered in regards to our use of the CPT with children, including concerns expressed by our Research Ethics Board (REB). We will share how we have addressed these issues in our work and with our IRB. We will also present empirical data from surveys with children, parents, and researchers regarding the acceptability of the CPT. Our findings show that adverse events are rare and transient amongst children who undergo the $\mathrm{CPT}$ and that the majority of researchers, children, and parents report positive experiences with the CPT. We conclude that the CPT is ethically permissible in pain research with children, provided there are developmentally appropriate study safeguards.

\section{B}

\section{DECEPTION IN EXPERIMENTAL HUMAN PAIN RESEARCH: IS THERE AN ALTERNATIVE?}

\section{Joel Katz}

York University, Toronto, Ontario

Use of deception violates the principles of human experimentation put forward by the world's leading regulatory bodies on research ethics. Deception (1) denies participants their legal and ethical right to informed consent by failing to disclose relevant information or by providing patently false information; (2) may manipulate individuals to participate in research that they otherwise would not have; and (3) may cause distress and lack of trust in research once the deception has been revealed. Nevertheless, psychologists and other scientists routinely use deception in their experimental studies. Use of deception is justified on the grounds that (1) the knowledge gained is worth the risks to the participants and (2) full disclosure would influence the participants' responses and invalidate the results. As such, deception is seen as an imperfect, but necessary means of ensuring the continuity of scientific progress and the validity of new scientific knowledge. I will discuss instances of deception in experimental human pain research in children and adults and present results using an alternative approach to the informed consent process called "authorized deception".

\section{C}

\section{ETHICS OF ANIMAL PAIN RESEARCH: A PERSONAL} PERSPECTIVE

Jeffrey S Mogil

McGill University \& Alan Edwards Centre, Montreal, Quebec

The intentional infliction of pain on laboratory animals is a particularly hot-button ethical issue, both from a philosophical and regulatory perspective. I will discuss the continuing necessity of pain research on animals, issues of regulation of animal use, challenges to implementation of the " 3 Rs" (replacement, refinement, reduction), and advances in the recognition and treatment of incidental animal pain. Finally, I will relate a personal experience with an ethical situation involving animal pain testing, and its ironic aftermath.

\section{SESSION $102-10: 30$ AM}

\section{3}

\section{SAFE AND EFFECTIVE PRESCRIBING CONTROLLED SUBSTANCES FOR PAIN MANAGEMENT: STRATEGIES FROM THE OFFICE TO THE COMMUNITY}

Chair: Hance Clarke

Department of Anesthesia and Pain Management, University of Toronto, Toronto, Ontario

Speakers: Peter MacDougall; John Fraser; Rhea MacDonald WORKSHOP OBJECTIVE:

1) Review the current literature as it pertains to the process of controlled substance prescribing and boundary setting in this context.

2) Review the current literature regarding opioid and controlled substance monitoring. 
3) Provide practical strategies for prescribing process, boundary management and monitoring of controlled substance prescribing.

4) Describe a practical strategy for management of controlled substance prescribing as a community.

Learning Objectives:

1. Participants will gain an understanding of the concepts of healthy prescribing process and boundary management as they relate to controlled substance prescribing.

2. Participants will learn appropriate strategies to develop monitoring processes for opioid use/abuse in the management of chronic non-cancer pain.

3. Participants will have a better understanding of the need to develop community strategies to manage controlled substance prescribing.

\section{$3 A$}

\section{SAFE AND EFFECTIVE PRESCRIBING OF OPIOIDS AND CONTROLLED SUBSTANCES: PROCESS AND BOUNDARIES} Peter MacDougall

Department of Anesthesia, Pain Management and Peri-operative Medicine, Dalhousie University; Nova Scotia Chronic Pain Collaborative Care Network, Halifax, Nova Scotia

Prescribing opioid medications for chronic non-cancer pain has been associated with significant morbidity and mortality (1). Safe prescribing of these medications requires both clear process and boundaries for the management of the patient. The Canadian Guidelines Opioid prescribing for Chronic Non-Cancer pain (2) provides a framework for prescribing of these medications. These guidelines outline a clear process for assessment of the risk of addiction, a trial of opioid prescribing, monitoring and, when necessary, withdrawl of the medications.

In addition to prescribing process, it is important to discuss with the patient the boundaries within which the medications will be prescribed. The maintenance of prescribing boundaries is a teachable skill crucial to the healthy physician patient relationship.

This portion of the workshop will provide a review of the current literature regarding opioid prescribing process and boundary development. We will discuss effective process for prescribing opioids in the setting of Chronic Non-Cancer Pain as well as techniques for the development of effective boundaries in the context of opioid prescribing.

\section{B \\ EFFECTIVE MONITORING OF CONTROLLED SUBSTANCE PRESCRIBING}

John Fraser

Pain Management Unit, QEII Health Sciences Center, Halifax, Nova Scotia

The goal of patient monitoring is to: (i) identify risk factors for opioid misuse, (ii) identify behaviors associated with opioid misuse - Aberrant Opioid Behaviors (AOBs) and to identify misuse of opioids or other addictive substances. This session will provide a review of the current literature regarding opioid misuse and opioid monitoring. The Canadian Opioid Guidelines (1) provided a number of recommendations for patient monitoring. As in any patient interaction, monitoring must be tailored to the individual patient needs. We will discuss techniques for identifying risks including the careful addiction history and tools such as the Opioid Risk Tool. Evidence suggests that the presence of $\mathrm{AOB}$ in the setting of abnormal urine screening is a better predictor of opioid misuse than AOB or urine screen alone. This portion of the symposium will discuss the development and implementation of a novel $\mathrm{AOB}$ tracking tool. In addition, the use of urine drug screening in the setting of chronic non-cancer pain will be discussed. The pros and cons of regular urine screening as well as the fallibilities of the testing will be highlighted. Finally, suggestions for developing a comprehensive patient monitoring program will be discussed.

\section{$3 \mathrm{C}$}

\section{A COMMUNITY APPROACH TO MANAGEMENT OF PRESCRIBING CONTROLLED SUBSTANCES \\ Rhea MacDonald}

Inverness Medical Clinic, Inverness, Nova Scotia

Prescribing controlled substances in small communities affected by problems of addiction to prescription controlled substances face unique challenges. One of the most vexing problems is lack of consistent prescribing practice among members of the medical community. Such problems are compounded by sudden loss or departure of members of the physician community and the need to absorb the newly orphaned patients into existing practices.

This portion of the workshop will present the story of a community faced with this 'perfect storm' problem. The medical community came together as a united community to develop a plan for addressing the burgeoning problem of controlled substance prescribing in the context of the impending retirement of a long term physician. The presentation describes how the community as a whole reached out to identify and engage available resources. The resources included the Nova Scotia Chronic Pain Collaborative Care Network, the Nova Scotia Prescription Monitoring Program, the College of Physicians and Surgeons of Nova Scotia, pharmacists and law enforcement. The medical community developed and implemented a cohesive plan to manage prescribing as a community. They agreed to and implemented a consistent approach to assessment, monitoring and prescribing. This type of broad community engagement and action serves as a template for other communities to manage challenging health care issues such as addictions.

\section{REFERENCES:}

1. Kuehn BM. Opioid prescriptions soar: increase in legitimate use as well as abuse. JAMA. 297(3):249-51, 2007

2. http://nationalpaincentre.mcmaster.ca/opioid/

\section{SESSION $103-10: 30$ AM}

\section{4}

\section{CONSIDERING CULTURAL CONTEXT OF PAIN IN ABORIGINAL PEOPLE: LINKING REALITY, RESEARCH AND EDUCATION}

\section{Chair: Margot Latimer}

Speakers: Margot Latimer; Sam Wong; Katherine Harman WORKSHOP OBJECTIVE:

The aim of this inter-professional symposia is threefold: to share with audience members historical and cultural information about Aboriginal people which may influence their conceptualization and expression of pain; to share research related to understanding how Aboriginal people express pain; and to review education strategies to enhance clinician knowledge related to providing pain care to Aboriginal people.

Learning Objectives:

1. To understand the role historical events and cultural perspectives may play on Aboriginal peoples' expression of pain and subsequent pain-related health conditions.

2. Become more confident in understanding how Aboriginal peoples' pain may be interpreted by clinicians.

3. Become familiar with a sample of post-secondary education strategies that have been piloted in inter-professional and medical schools to enhance student understanding of health issues in Aboriginal people and the need to develop heightened pain care skills.

\section{A}

\section{CULTURAL AND HISTORICAL CONSIDERATIONS IN PAIN CARE FOR ABORIGINAL PEOPLE-WHAT DO WE KNOW? Margot Latimer}

\section{Dalhousie University, IWK Health Centre, Halifax, Nova Scotia}

Pain assessment is dependent upon effective communication and interpretation of the pain experience. Pain is a multidimensional phenomenon and how it is conceptualized is influenced to a large extent by one's cultural and past experiences. If not familiar with the cultural characteristics that define one's pain experiences, inaccurate and harmful responses may occur and yet there is little to no research in this area in Aboriginal children/youth. Research indicates that Aboriginal children and youth experience pain at a higher rate than non-Aboriginal children and this alarming difference persists into adulthood, with higher rates for many pain-related conditions reported. It is known that pain, if left untreated, can interfere with the achievement of a productive, healthy life. Untreated pain may be even more profound and result in even poorer outcomes amongst Aboriginal people given that high rates of ill health and resource inequities are a 
reality amongst these groups. Further, there is some indication that Aboriginal people do not express pain in the manner expected by nonAboriginal care providers and that this may contribute to higher rates of under assessment, under treatment and subsequent pain prevalence. This session will include a brief overview of historical events and cultural traditions that may shape Aboriginal peoples' expression and experience of pain. We will present results from a study conducted in a large First Nation Community identifying discrepant perspectives related to pain expression and clinician interpretation and the community-based steps to address this gap.

\section{B \\ MANY HANDS ONE DREAM: CANADIAN PEDIATRIC SOCIETY RESIDENT TRAINING INITIATIVE TO ENHANCE ABORIGINAL WELLNESS}

\section{Sam Wong}

University of Alberta, Canadian Pediatric Society, Edmonton, Alberta

Although the Aboriginal population accounts for only 3.3\% of Canada's total population, Aboriginal children represent $5.6 \%$ of all children in Canada. Despite this, they lag far behind other Canadian children when it comes to their health. The Canadian Paediatric Society (CPS) is one of more than 11 national organizations involved in developing a long-term initiative called the 'Many Hands, One Dream' to reduce the health inequities facing Aboriginal people including First Nations, Inuit and Métis children and youth in Canada. The goal of Many Hands, One Dream is to build a new vision of health that has children, youth and families at its core. Included in this is a one-and-a-half day medical resident workshop delivered in academic centres across Canada by CPS members with experience and expertise in caring for First Nations, Inuit and Métis children and youth. This session will review this CPS initiative as it relates to pain care in Aboriginal children in terms of history, culture, jurisdictional policy and health inequities and training of medical residents at universities across Canada. We will present initiative strategies such as training videos and content as well as student evaluations.

\section{C}

\section{UNIVERSITY-BASED INTER-PROFESSIONAL HEALTH EDUCATION INITIATIVE: CONSIDERING CULTURE AND PAIN IN ABORIGINAL PEOPLE}

\section{Katherine Harman}

\section{Dalhousie University, Halifax, Nova Scotia}

We used an Inter-professional Health Education (IPHE) initiative to create a learning space to enhance student understanding of the cultural impact on pain expression and interpretation. This session will review a university-based IPHE mini-course created to address the learning needs of undergraduate and graduate students related to pain care in Aboriginal people. Health professions' students from different faculties including: nursing, health and human performance, occupational therapy, pharmacy and social work completed a three-week ( 3 hours/week) mini course. We used a case-based approach to expose students to the background presented by Dr. Latimer. Students were required to work through the case considering cultural and historical factors and how pain care may need to be integrated into the perspectives of Aboriginal peoples. In addition to the casework faculty also introduced students to relevant research and the CPS content as well as attributes of inter-professional health education (IPHE) through readings; group work, dynamic (interactive) lectures and role-play scenarios. Multiple strategies were used to support students to learn how to communicate and translate their knowledge about pain into practice, and to learn to listen to Aboriginal patients regarding their experiences of pain. Pre-post curriculum evaluations will be presented.

\section{SESSION $104-1: 30$ PM}

5

\section{EFFECTIVELY TRANSLATING YOUR RESEARCH TO OTHER RESEARCHERS, CLINICIANS, AND THE PUBLIC \\ Chair: Kathryn A Birnie \\ Speakers: Jeffrey Mogil; Rob McEwan; Mary Lynch \\ WORKSHOP OBJECTIVE:}

Given the commonplace experience of pain, new findings in pain research often generate great media attention and high public interest. Furthermore, with advancing technologies, there are increasing opportunities to share research findings through both formal and informal avenues. However, pain research is often highly specialized. It is our responsibility as pain researchers to make our work understandable, interesting, and relevant to others. The aim of this trainee-oriented symposium is to offer guidance and skills to clinical and basic science trainees for effectively sharing their research with multidisciplinary researchers, clinicians, the media, and the public. Discussion amongst attendees will be encouraged.

Learning Objectives:

1. To learn strategies for effectively presenting scientific research to multidisciplinary audiences of researchers and clinicians.

2. To gain awareness and skills for effectively communicating scientific findings to the media and the public.

3. To increase exposure to the role pain researchers and clinicians can play in advocating for better pain management within the field, with politicians, and with the public.

\section{$5 \mathrm{~A}$}

\section{BREAKING FREE OF THE LAB: TIPS FOR TRANSLATING RESEARCH TO THE WIDEST POSSIBLE AUDIENCE Jeffrey Mogil \\ McGill University, Montreal, Quebec}

Scientists, especially young scientists, have a tendency to get caught "in the trees" when giving presentations about their research, even as the vast majority of the audience is only interested in "the forest". I will discuss common mistakes in presenting research - both in substance and style and how to avoid them, and strategies for better tailoring of research talks to the likely audience.

\section{B}

\section{CUTTING THROUGH THE CLUTTER: PROMOTING YOUR MESSAGES TO THE MASSES}

Rob McEwan

Argyle Communications

We live in a 24-hour news cycle of round-the-clock reporting. The newsroom doesn't sleep, you hold a printing press in the palm of your hand and you have 140 characters to qualify your idea to the world. Every presentation, public appearance, and interview is an opportunity for your ideas, research, and messages to gain acceptance and recognition. Our media landscape, the importance of social media, and tips to engage the press in a meaningful way will be discussed.

\section{$5 \mathrm{C}$}

\section{PAIN ADVOCACY IN A PAIN HATING WORLD}

Mary Lynch

Dalhousie University \& QEII Health Sciences Centre, Halifax, Nova Scotia

This presentation will journey through the real world experience of the presenter who has worked in the field of pain for 25 years. In assisting people with pain conditions the clinician learns very early that, in addition to suffering with pain, the patient is stigmatized and often accused of malingering or of being a 'drug seeker'. Tragically, it is a frequent experience to witness the inadequate treatment of pain in inpatient care. This experience compels one to become an advocate. This presentation will discuss advocacy in pain using many modes of communication, including peer-reviewed publications that dispel myths about pain, to written and electronic media. Strategies for identifying key messages and using media opportunities to get these key messages across will be reviewed. 


\section{SESSION $105-1: 30$ PM}

\section{6}

\section{THE SIDE EFFECTS OF OPIOID USE: FROM GENES AND CELLS TO THE HUMAN PATIENT}

Chair: Tuan Trang

Speakers: Tuan Trang; Catherine Cahill; Lori Montgomery WORKSHOP OBJECTIVE:

Opioid drugs are critical in pain therapy but their use is plagued with debilitating side effects, such as analgesic tolerance, opioid induced hyperalgesia, and drug dependence. This session focuses on new discoveries made in understanding the cellular processes that are responsible for the unwanted side effects of opioid use and the clinical translation of these discoveries at the level of molecules and cells to the human patient.

Learning Objectives:

1. Understand the cellular targets of opioid drugs in the central nervous system and their role in producing the side effects associated with opioid use.

2. Appreciate the mechanistic relationship between opioid induced hyperalgesia and tolerance.

3. Recognize the clinical significance of the unwanted side effects of opioid use in patients.

\section{A}

\section{THE X FACTOR: P2X4 RECEPTORS IN OPIOID THERAPY}

\section{Tuan Trang}

\section{University of Calgary, Calgary, Alberta}

The management of chronic pain is a major clinical challenge because it is difficult to treat, and even with treatments that do provide some level of pain relief there is a high incidence of disabling side effects. Opiates such as morphine remain the gold standard pain relieving drugs but their use is plagued with debilitating side effects. Two main problems that limit the treatment of pain with opiates are: opioid induced hyperalgesia (a paradoxical increase in pain sensitivity) and tolerance (diminished effects such that higher doses are needed to produce the same level of pain relief). The prevailing dogma is that both of these problems are inevitable consequences of opiate use, and that tolerance and hyperalgesia reflect a single underlying mechanism. We have recent evidence that dispels this view by uncovering that morphine hyperalgesia, but not tolerance, is mediated by P2X4 receptor initiated microglia-to-neuron signalling that increases activity of pain-transmitting neurons in the spinal cord. By teasing apart the entire core sequence of signalling events and molecules, we dissociated the critical molecular and cellular mechanisms of morphine hyperalgesia from that of tolerance.

\section{B}

\section{MODULATION OF OPIOID TOLERANCE AND HYPERALGESIA BY ULTRA-LOW DOSE NALOXONE OCCURS INDEPENDENTLY OF TOLL-LIKE RECEPTOR-4}

\section{Catherine Cahill}

\section{University of California Irvine, Irvine, California, USA}

Ultra-low doses (ULD) of the opioid receptor antagonists block the induction of opioid analgesic tolerance and reverse established tolerance. Since opioids have been shown to bind to the Toll-like receptor 4 (TLR-4) in a non-stereo-selective manner and activation of TLR-4 triggers gliosis, we investigated the specific role of TLR-4 in opioid tolerance and hyperalgesia. Adult male $\mathrm{C} 3 \mathrm{H} / \mathrm{HeOuJ}$ (WT) or $\mathrm{C} 3 \mathrm{H} / \mathrm{HeJ}(\mathrm{TLR}-4 \mathrm{KO}$ ) mice were administered morphine or combined with stereoisomers of naloxone (NLX), once daily for five days. No difference was observed in acute morphine (MS) analgesia between the WT and TLR-4 KO mice. Following 5 days of MS administration, the degree of analgesic tolerance and opioidinduced hyperalgesia was not significantly different between WT and KO mice. Systemic administration of (-)NLX attenuated the development of tolerance in WT mice and KO mice. The ULD opioid antagonist effect was stereo-selective in the tolerance study as (+)NLX (opioid receptor inactive) did not attenuate tolerance in WT or $\mathrm{KO}$ mice compared to controls treated with MS only. However, both isomers were able to attenuate opioid-induced hyperalgesia and this effect was also present in the TLR-4 KOs. In conclusion, the stereo-selectivity of the ULD effect on tolerance suggests that opioid antagonists do not modulate opioid tolerance via TLR-4 as hypothesized, thus, an alternate mechanism of action must be responsible for the prevention and reversal of analgesic tolerance by ULD opioid antagonists. The ability of both NLX isomers to inhibit opioid-induced hyperalgesia is interesting, however the mechanism does not appear to be mediated via modulation of the TLR-4 protein.

\section{C}

\section{MANAGING ADVERSE EFFECTS OF OPIOID USE IN THE} CHRONIC PAIN PATIENT

\section{Lori Montgomery}

University of Calgary, Calgary, Alberta

Basic scientists are beginning to unravel the mysteries surrounding the development of opioid induced hyperalgesia, tolerance and dependence, and there is promising evidence that we may soon be able to mitigate those effects in the patient. However, in the meantime, they remain some of the most common reasons for opioid therapy to be discontinued. Even more alarmingly, if not addressed, they are the root of dangerous dose escalation that can lead to morbidity and mortality. This session will survey the clinical literature in the area, and provide practical tips for managing long term adverse effects of opioid therapy.

\section{SESSION $106-1: 30$ PM}

\section{7}

WHEN “JUST DO IT" JUST WON'T DO: EXPLORING THE COMPLEX RELATIONSHIPS BETWEEN PHYSICAL ACTIVITY

\section{AND PAIN}

Chair: Susan M Tupper

Speakers: Susan M Tupper; Nancy C Gyurcsik; Brian W Timmons WORKSHOP OBJECTIVE:

Regular physical activity participation is frequently prescribed as a key treatment recommendation for individuals living with persistent pain conditions. However, this population faces unique obstacles when engaging in and maintaining activity programs. This workshop will explore barriers to physical activity adherence, and the short and long-term relationships between activity, inflammation and pain in diverse clinical populations. Findings from lab-based, survey, observational and interventional research will be presented.

Learning Objectives:

1. Describe the short-term relationship between daily activity, sedentariness, and pain in youth with chronic pain conditions.

2. Describe relationships between theory-based activity-specific and diseasespecific beliefs and adherence to the recommended dose of physical activity for arthritis management.

3. Describe the relationship between physical activity and biomarkers of inflammation in children with a chronic inflammatory disease.

\section{$7 \mathrm{~A}$}

THE SHORT TERM RELATIONSHIP BETWEEN ACTIVITY, INACTIVITY AND PAIN FOR YOUTH WITH CHRONIC PAIN CONDITIONS

Susan M Tupper

University of Saskatchewan, Saskatoon, Saskatchewan

The relationship between pain and activity is complex and bidirectional. Children and adolescents with chronic pain conditions are less physically active and have lower physical function than their healthy peers. Although pain has been identified as a possible barrier to physical activity participation in this population, regular exercise is recommended as an important therapeutic intervention for long term pain reduction. A study in which the short-term relationship between pain intensity and daily observed physical activity and sedentariness in youth with persistent pain conditions will be reported. In this study, an electronic diary application (app) for the iPod Touch was developed to collect momentary pain data 7 times per day for 4 days from 28 youth age 8 to 17 years with juvenile idiopathic arthritis (JIA; $n=11)$ or non-arthritic pain conditions $(n=17)$. Physical activity data was collected by hip mounted accelerometers continuously over the sampling period. Generalized estimating equation 
analysis was conducted to examine the short-term relationship between pain intensity and activity level. There was a $U$ shaped short-term relationship between pain intensity, activity and sedentariness for youth with JIA and non arthritic persistent pain. Possible implications for exercise prescription and future directions for physical activity research in this population will be discussed. Preliminary findings from one interventional study and one cohort study on pain in youth with juvenile idiopathic arthritis will be summarized.

\section{B}

IT'S NOT ONLY ABOUT THE PAIN: UNDERSTANDING THE ROLE OF ACTIVITY-SPECIFIC AND DISEASE-SPECIFIC BELIEFS IMPORTANT FOR PHYSICAL ACTIVITY ADHERENCE FOR BETTER ARTHRITIS SELF-MANAGEMENT Nancy C Gyurcsik

University of Saskatchewan, Saskatoon, Saskatchewan

Most adults with arthritis do not adhere to the recommended dose of physical activity (PA) for disease management (150+ minutes/week). Although people often report pain as a barrier to their PA, it rarely predicts PA adherence. Other factors, including social cognitive beliefs, may be important in influencing whether people adhere to PA. The purpose is to summarize findings from a research program investigating relationships between activity- and disease-specific beliefs related to PA adherence. Research program participants were adults with arthritis $(n=300+)$ who completed online surveys of demographics, activity- and disease-specific beliefs, and PA over time. Across studies, the activity-specific beliefs of self-regulatory efficacy (SRE) to overcome barriers (e.g., stiffness, pain, swollen joints) and SRE to schedule and plan activity were reliably predictive of PA, both when participants were in an arthritis flare or when not in a flare. Pain acceptance, involving a willingness to engage in PA despite pain, was a disease-specific belief that distinguished participants who met the recommended PA dose compared to those who did not. Pain intensity remained an inconsistent predictor of PA. Findings offer a clearer description of factors related to adherence. People who accept their pain and who have high SRE to overcome barriers and to schedule and plan activity are best able to adhere to PA for disease management. Discussion will focus on future research directions, including investigations of other disease-specific factors, such as arthritis-related pain anxiety, that complement activityspecific beliefs, eventually leading to effective interventions to stem the problem of PA non-adherence.

Funded by SSHRC grant \#410-2005-2292 and SHRF-CIHR-RPP grant \#122108

\section{C}

\section{IS PHYSICAL ACTIVITY AN ANTI-INFLAMMATORY THERAPY FOR CHILDREN WITH A CHRONIC INFLAMMATORY DISEASE?}

Brian W Timmons

\section{McMaster University, Hamilton, Ontario}

AIMS: Describe the effects of specific episodes of exercise on biomarkers of inflammation. Describe the relationship between daily physical activity and biomarkers of inflammation. Discuss the role of physical activity as an anti-inflammatory therapy for children with a chronic inflammatory disease.

METHODS: Children with a diagnosis of juvenile idiopathic arthritis (JIA), Crohn's disease (CD), or cystic fibrosis (CF) were invited to participate in a series of studies to understand the effects of exercise on inflammation. Each participant completed 2 different types of exercise with blood samples collected over a 3 -h period to characterize changes in key biomarkers of inflammation, such as IL- 6 and TNF- $\alpha$. Each participant also wore an accelerometer for 7 consecutive days so that the relationships between levels of daily physical activity and resting levels of inflammation could be determined.

RESULTS: Specific episodes of exercise created similar changes in biomarkers of inflammation among children with JIA, CD, or CF that were consistent with an anti-inflammatory environment. These changes were consistent with the response of healthy children who served as a control group. We found an inverse relationship between biomarkers of inflammation and levels of daily physical activity assessed using objective monitoring. Children with JIA, CD, or CF who were the least active also experienced the highest levels of CRP, IL-6, and TNF- $\alpha$.

CONCLUSIONS: Specific episodes of exercise can create an antiinflammatory environment for children with a chronic inflammatory disease. The cumulative effects of greater activity seem to result in an overall reduction in systemic inflammation. These findings will be interpreted in light of safe and effective exercise recommendations for children with JIA, CD, or CD.

4:15 pm INTERPROFESSIONAL PAIN EDUCATION PRESENTATION

8

Chair: Mary E Lynch

Dalhousie University, Halifax, Nova Scotia

8A

\section{THE LONG AND WINDY ROAD OF PAIN-IPE EDUCATION DEVELOPMENT}

\section{Judi Hunter}

CPS Interprofessional Pain Education Award Recipient 2013

Departments of Physical Therapy, University of Alberta and

University of Toronto, Curriculum Director, University of Alberta

Certificate in Pain Management

Learning Objectives:

By the end of this session participants will:

1. Consider the breadth and depth of the meaning of IPE.

2. Consider the experiences of applying IPE theory to improve collaborative pain-care amongst clinicians who practice across the continuum of pain management settings.

3. Reflect on the joys and frustrations of maintaining awareness of stay aware of other's perspectives.

The practical aspects of developing pain-IPE is an exciting area, promising improved care for individuals' acute or chronic pain. The evidence for the best way to bridge the chasm between knowledge and collaborative pain management was essentially lacking only a decade ago. "Iterative" curriculum development was, and still is, a key strategy. I plan to take a lighthearted look my experiences along the way - and present exciting evidence for the future.

THURSDAY MAY 9, 2013
SYMPOSIA - 7:30 AM

\section{9}

\section{OSTEOARTHRITIS PAIN - EVOLVING CONCEPTS}

Chair: Jason J McDougall

Departments of Pharmacology and Anaesthesia, Dalhousie

University, Halifax, Nova Scotia

Speakers: Serge Marchand; Philip A Baer

Learning Objectives:

1. Review the evolving basic science of osteoarthritis pain.

2. Describe how OA pain can become centralized.

3. Translate basic science into practical clinical pain management.

\section{A}

\section{OA PAIN - FROM NOCICEPTION TO CENTRAL} SENSITIZATION

Serge Marchand

Faculté de médecine, Université de Sherbrooke, Centre de recherche clinique Étienne-Le Bel du CHUS, Sherbrooke, Québec Osteoarthritis (OA) is a frequent and important chronic pain problem resulting from the interaction of nociceptive and central sensitization mechanisms. During this presentation, I will introduce the neurophysiological mechanisms implicated in the development and persistency of $\mathrm{OA}$ pain and how central sensitization can be maintained by the continuous nociceptive activity. We will also discuss the role of endogenous excitatory 
and inhibitory mechanisms in chronic pain such as OA and the importance of understanding these mechanisms in adapting the treatments.

\section{B}

\section{OA PAIN - DIAGNOSIS AND CLINICAL MANAGEMENT}

\section{Philip A Baer}

Editor-in-Chief, Journal of the Canadian Rheumatology Association, Chair, Section of Rheumatology, Ontario Medical Association, Scarborough, Ontario

Osteoarthritis (OA) is the most common form of arthritis, affecting over 4 million Canadians at tremendous personal and societal cost. It is a leading cause of disability. Pain and stiffness are the cardinal symptoms, leading to loss of function and often associated mood disturbance. OA can be diagnosed clinically without expensive investigations. Numerous OA Guidelines have been published in recent years. This presentation will focus on the 2012 American College of Rheumatology OA guidelines, which benefited from significant Canadian input. Emphasis will be on treatment with combination non-pharmacologic and pharmacologic therapies, addressing multiple pain pathways.

\section{KEYNOTE SPEAKERS - 8:45 AM}

\section{0}

\section{Chair: Laura S Stone}

Alan Edwards Centre for Research on Pain, Faculty of Dentistry, McGill University, Montreal, Quebec

Speakers: A Vania Apkarian; Gary J Bennett

\section{A}

BRAIN LEARNING MECHANISMS AND TRANSITION TO CHRONIC PAIN

Speaker: A Vania Apkarian

Physiology, Northwestern University, Chicago, Illinois, USA

Learning Objectives:

1. Evidence that to date the best predictors of transition to chronic pain are brain anatomy and brain physiology.

2. Brain learning circuitry, the mesolimbic reward/aversion system, is intimately involved in transition to chronic pain.

3. Understanding the molecular mechanisms of this brain circuitry can lead to novel therapy approaches for at least preventing transition to chronic pain.

\section{$10 B$}

\section{MITOTOXICITY AS THE CAUSE OF CHEMOTHERAPY- INDUCED PERIPHERAL NEUROPATHY (CPN)}

Speaker: Gary J Bennett

Professor \& Canada Senior Research Chair, Department of Anesthesia, Faculty of Dentistry, and the Alan Edwards Center for Research on Pain, McGill University, Montreal, Quebec

Chemotherapeutics in the vinca alkaloid, taxane, platinum-agent, and proteasome-inhibitor classes all produce a chronic distal symmetrical sensory neuropathy that is often accompanied by neuropathic pain. Even though these drugs have distinctly different anti-cancer mechanisms of action, clinical accounts suggest that these chronic neuropathies are very similar. Work with rat models of paclitaxel-, oxaliplatin-, and bortezomib-induced CIPN show that the chronic sensory neuropathies are nearly identical. In the rat, each of these agents causes an increase in the incidence of swollen and vacuolated mitochondria in peripheral nerve A-fiber and C-fiber primary afferent axons, but not in their Schwann cells. Direct measurements of mitochondrial function in peripheral nerve preparations from rats with confirmed CIPN show chronic dysfunction in mitochondrial respiratory Complexes I and II, and in ATP production. Thus, mitotoxicity is likely to be the fundamental cause of CIPN. A chronic drug-induced mitochondrial injury results in an energy deficiency that results in spontaneous afferent discharge and degeneration of intraepidermal nerve fibers (IENFs). Similarly, a mitotoxic effect of excess glucose may be responsible for painful diabetic neuropathy. Learning Objectives:

1. To recognize the clinical presentation of chemotherapy-induced peripheral neuropathy (CIPN).
2. To evaluate the laboratory evidence concerning the mitotoxicity hypothesis for the cause of CIPN.

3. To be aware of the therapeutic implications of the mitotoxicity hypothesis.

\section{SESSION $201-11: 00$ AM} DATA BLITZ

\section{$11 \mathrm{~A}$}

DOES THE IMPLEMENTATION OF A NURSE PRACTITIONER-LED PAIN MANAGEMENT TEAM IMPROVE RESIDENT OUTCOMES IN LONG TERM CARE?

Sharon Kaasalainen ${ }^{1}$; Noori Akhtar-Danesh ${ }^{1}$; Kevin Brazil ${ }^{2}$; Faith Donald ${ }^{3}$; Ruth Martin-Misener ${ }^{4}$; Alba DiCenso ${ }^{1}$; Thomas Hadjistavropoulos ${ }^{5}$; Lisa Dolovich ${ }^{1}$; Carrie McAiney ${ }^{1}$; Deboral Marshall ${ }^{6}$

${ }^{1}$ McMaster University, Hamilton, Ontario; ${ }^{2}$ Queen's University, Belfast, United Kingdom; ${ }^{3}$ Ryerson University, Toronto, Ontario; ${ }^{4}$ Dalhousie University, Halifax, Nova Scotia; ${ }^{5}$ University of Regina, Regina, Saskatchewan; ${ }^{6}$ University of Calgary, Calgary, Alberta AIM: This study evaluated the effectiveness of implementing a Nurse Practitioner (NP)-led, inter-professional (IP) pain management team in long-term care (LTC) team on improving (a) resident outcomes (i.e., pain, functional status, agitation, depression); (b) clinical practice behaviours (i.e., documentation of pain assessments, use of non-pharmacological and pharmacological interventions); (c) quality of pain medication prescribing practices; and (d) IP collaboration. We also obtained some preliminary information on the costs of implementing this approach.

METHODS: Using a controlled before-after study, six LTC homes were allocated to one of three groups: 1) an NP-led Pain Team; 2) NP but NO pain management team; or, 3) no NP, no pain management team. Over the one-year intervention period, there was a $32 \%$ dropout rate for the full intervention group, Group 1 (45/139), 30\% dropout rate for the partial intervention group (33/108), and a 25\% dropout rate for the control group (24/98). RESULTS: Implementing an NP-led Pain Team in LTC significantly reduced residents' pain and improved functional status compared to usual care without access to an NP. Positive changes in clinical practice behaviours (e.g., assessing pain, developing care plans related to pain management, documenting effectiveness of pain interventions) occurred over the intervention period for both the NP-led Pain Team and NP-only groups; these changes did not occur to the same extent, if at all, in the control group.

CONCLUSIONS: The findings from this study showed that implementing an NP-led Pain Team can significantly improve clinical practice behaviours of LTC staff and improve resident pain and functional status. LTC homes should employ an NP, with preference for the NP being situated onsite as opposed to an offsite consultative role, to facilitate more consistent and timely access to pain management.

\section{$11 B$}

DEXMEDETOMIDINE AS AN EFFECTIVE ADJUVANT FOR PAIN RELATED TO INFUSION OF CHIMERIC ANTIBODY; A CASE SERIES

Gillian Lauder ${ }^{1}$; Rebecca Deyell ${ }^{2}$; Matthias Görges ${ }^{3}$; Michele Smith $^{1}$; Nicholas West ${ }^{4}$; Pamela Winton ${ }^{5}$

${ }^{1}$ Department of Anesthesia; ${ }^{2}$ Department of Oncology, British Columbia Childrens Hospital (BCCH); ${ }^{3}$ Department of Electrical and Computer Engineering; ${ }^{4}$ Department of Anesthesiology, Pharmacology and Therapeutics, University of British Columbia, Vancouver, British Columbia; ${ }^{5}$ Royal Hospital for Sick Children, Edinburgh, United Kingdom

AIM: Targeted immunotherapy using Chimeric Monoclonal Anti-GD2 antibody Ch14.18 is a novel, targeted therapy for children with neuroblastoma and is associated with significant pain [1]. Analgesic treatment with a high-dose opioid infusion is recommended [2], as adjunct analgesic agents are restricted in oncology patients. We present a case series in which dexmedetomidine was used as adjunct to hydromorphone infusion during Ch14.18 therapy in the oncology ward setting.

METHODS: The Ch14.18 infusion was administered for a minimum of 10 hours daily. One hour before starting Ch14.18, dexmedetomidine 
( 0.1 to $0.6 \mathrm{mcg} / \mathrm{kg} / \mathrm{hr}$ ) and hydromorphone ( 2 to $8 \mathrm{mcg} / \mathrm{kg} / \mathrm{hr}$ ) infusions were commenced, with strict monitoring and nursing policies. Dexmedetomidine was discontinued 1 hour after Ch14.18 completion. Hydromorphone was then weaned as tolerated. Patient charts were reviewed for infusion details and documented occurrences of clinically significant pain, hypotension, oxygen desaturation and oversedation.

RESULTS: Four consecutive patients, median age 2 years (range 2 to 12 years) received dexmedetomidine and hydromorphone infusions during five 4-day cycles of Ch14.18 therapy. Detailed results will be presented. Overview of results shows that dexmedetomidine facilitates pain mangagement with doses of hydromorphone in a normal range and minimal occurrences of clinically significant pain, hypotension, oxygen desaturation and oversedation. Hydromorphone and dexmedetomidine could be easily titrated down without resurgence of pain at the end of each Ch14.18 infusion.

CONCLUSIONS: Dexmedetomidine infusion is an effective and safe pain management adjunct to parenteral opioids for Ch14.18 therapy in the ward setting

REFERENCES:

1. Yu et al. N Engl J Med 2010;363:1324-34

2. Saleh et al. Hum Antibodies Hybridomas 1992;3:19-24

\section{C}

EXAMINING THE RELATIONSHIP BETWEEN CHRONIC PAIN AND HEALTH RELATED QUALITY OF LIFE AMONG OLDER CANADIAN ADULTS WITH DISABILITY

Kristina Zawaly ${ }^{1}$; Shahin Shooshtari ${ }^{2}$; Ian Clara ${ }^{3}$; Nancy Hansen ${ }^{1}$; Verena Menec ${ }^{4}$

${ }^{1}$ Interdisciplinary Program in Disability Studies; ${ }^{2}$ Faculty of Human Ecology; ${ }^{3}$ Manitoba Research Data Centre; ${ }^{4}$ Faculty of Medicine, University of Manitoba, Winnipeg, Manitoba

AIM: Chronic pain is a common health issue that is found to be associated with poor quality of life among older Canadians. Despite the vast literature on chronic pain and health-related quality of life (HRQoL), little literature exists on the effects of chronic pain on HRQoL among older Canadian adults with disability. Thus, the extent by which chronic pain affects HRQoL of older Canadians with disability is not well understood.

OBJECTIVES: This study was conducted to: 1) estimate prevalence of chronic pain among older Canadian adults with disability, 2) examine the relationship between chronic pain and HRQoL, 3 ) assess if there is a doseresponse relationship between chronic pain and HRQoL.

METHODS: This study was a secondary analysis of cross-sectional data from the 2006 Participation and Activity Limitation Survey (PALS). The main objective of PALS was to develop a comprehensive database of Canadians with disabilities. The survey target population consisted of those individuals living in private dwellings in the ten Canadian provinces and three Canadian territories, who reported an activity limitation in the Canadian Census. For the purpose of this study, those who were 55 years of age or older at the time of the survey were selected. In this study, chronic pain was measured using a derived variable, which is based on individual responses to the pain related screening questions in the survey. The single item self-rated health question in the survey was used to measure HRQoL. Weighted data was used to examine the relationship between chronic pain and HRQoL using bivariate and multivariate logistic regression, controlling for the effects of potential covariates. To fully account for the survey sampling design, the bootstrap weights were applied.

RESULTS: An estimated 68\% of older Canadian adults with disability reported having chronic pain (45\% less severe and $23 \%$ more severe). There was a statistically significant association between chronic pain and HRQoL. Multivariate regression analysis confirmed a significant independent effect of chronic pain on self-reported HRQoL. Those who reported severe chronic pain had 3.34 times greater odds of reporting negative HRQoL, relative to those who reported no chronic pain. Older Canadians with disability who reported having less severe chronic pain had 1.39 times greater odds of reporting negative HRQoL, relative to those who reported no chronic pain. It was also found that those individuals with agility and mobility disability had greater odds of reporting negative HRQoL when all other potential factors were controlled during the multivariate analysis (AOR 1.85 [95\% CI 1.39 to 2.47] and AOR 2.62 [95\% CI 1.91 to 3.61], respectively).
CONCLUSIONS: Chronic pain is a significant health issue for older Canadian adults with disability, which negatively affects their HRQoL. Our results highlight the importance of pain assessment and management for older adults with disabilities in general and in particular among those with limited communication abilities.

\section{D}

\section{PAIN TEACHING IN THE UNDERGRADUATE MEDICAL PROGRAM IN THE FACULTY OF HEALTH SCIENCES AT MCMASTER UNIVERSITY: A CURRICULUM REVIEW}

John E Centofanti; Jaclyn Gilbert; Claudia Gomez; Eugenia Poon; D Norman Buckley

Department of Anesthesia, McMaster University, Hamilton, Ontario AIM: In medical training, variability exists in the content and time devoted to teaching pain. Guidelines for medical schools' pain curriculum have been developed by The International Association for the Study of Pain (IASP). This study aimed to identify the pain-related teaching in the undergraduate medical curriculum at McMaster University, and categorize the content within the IASP curriculum criteria.

METHODS: Four investigators (JC, EP, JG, CG) reviewed McMaster's medical curriculum, focusing on formal pain teaching. Data collection included a retrospective review of all formal academic sessions over 20112012, using the online curriculum database. All tutorials, large group sessions (LGS), and licensing exam lectures were systematically reviewed by a minimum of two investigators. Outcome measures included the content of tutorial cases and lecture slides that focused on pain teaching. All content was measured against the IASP Curriculum Outline on Pain for medicine.

RESULTS: $12.1 \%$ of LGS presentations (26 of 215) were identified to include pain education. An average of $32.3 \%$ of those lectures' slides mentioned pain (range 1.7\%-100\%). IASP criteria was noted a total of 165 times in LGS, with majority from basic sciences $(n=47)$ and pain etiology $(n=35) .23 .8 \%$ of tutorial cases (38 of 160$)$ included pain-related content. IASP criteria were noted a total of 104 times within tutorials, with majority from pain etiology $(n=51)$ and basic sciences $(n=19)$.

CONCLUSIONS: Lectures and tutorials in McMaster's curriculum incorporated pain-related teaching $12 \%$ and $24 \%$ of the time, respectively. Most common IASP criteria addressed included basic sciences and pain etiology. Utilizing the IASP criteria as a guide would provide a more holistic approach in training future physicians to comfortably manage patients with acute and chronic pain issues.

\section{$11 \mathrm{E}$}

\section{FINAL VERSION OF THE QUESTIONNAIRE TO IDENTIFY KNEE SYMPTOMS (QUIKS): CREATED USING A MULTIDIMENSIONAL RASCH ANALYSIS APPROACH} Clayon Hamilton ${ }^{1}$; Bert Chesworth ${ }^{2}$

\section{${ }^{1}$ Graduate Program in Health and Rehabilitation Sciences;}

${ }^{2}$ Department of Epidemiology and Biostatistics, University of Western Ontario, London, Ontario

AIM: To (1) create the final version of the QuIKS using the multidimensional Rasch analysis approach, and (2) to establish the internal validity of each of the QuIKS four subscales independently.

METHODS: This study included participants $(n=146)$ with clinically diagnosed knee osteoarthritis with knee pain or knee pain with no knee osteoarthritis diagnosis. Rasch analysis was conducted on the full 13-item QuIKS using a multidimensional approach which accounted for its subscale structure. Rasch analysis was conducted on each of the four individual subscales using an unidimensional approach. These scales were assessed for overall fit to the Rasch model, disordered thresholds, item and person fit, local dependency, differential item function (DIF) by five different characteristics, unidimensionality, internal consistency relability, and targeting of sample. To meet Rasch model assumptions, some items were rescored or deleted.

RESULTS: The 13-item QuIKS did not fit the Rasch model. After rescoring disordered thresholds in 10 items and the deletion of one misfit item, the remaining 12 items formed a four subscale multidimensional scale. However, these 12-item 4-subscale QuIKS had a unidimensional common latent trait, which fitted and met all assumption of the Rasch model within this four subscales structure. Each separate subscale, except the Interpreting 
subscale, fitted the Rasch model on their initial evaluation. The same item misfitted both the full QuIKS and the Interpreting subscale. Each separately modified subscale fitted and met all assumptions of the Rasch model. Except, one medication subscale item had DIF that showed participants with bilateral knee problems consistently reported more medication usage.

CONCLUSIONS: A 12-item version of the QuIKS and a modified version of each separate individual subscales demonstrated their dominant latent trait was capable of providing interval level measurement. The full 12-item QuIKS and the individual scales are independent measures for discriminating the level of impact of pain symptoms among people experiencing knee pain with or without confirmed knee osteoarthritis. Thus, the 12-item QuIKS might be useful when creating pain management strategies for individuals with knee pain.

\section{$11 \mathrm{~F}$ \\ AN APPROACH TO BEHAVIOUR CHANGE IN A CLBP PHYSIOTHERAPY PROGRAM FOR INJURED MILITARY MEMBERS}

\section{Katherine Harman}

School of Physiotherapy, Dalhousie University, Halifax, Nova Scotia

AIM: This interview-based study was conducted to better understand the reasoning process of the physiotherapist running a Back to Fitness program and the approach she took when using behaviour change techniques (BCT). The research objective was to describe the approach used, how the physiotherapist used different techniques and what she was trying to achieve with her clients.

METHODS: After studying video recordings of the six-week (lecture and exercise class) rehabilitation program (Back-to-Fitness), the lead physiotherapist was interviewed for this study. Video samples of the program were selected and 56 brief (longest was 2:11) Quick Time ${ }^{\mathrm{TM}}$ videoclips were created. Prior to the interview, the videoclips were sent to the physiotherapist to review. After informed consent was obtained, an audio- and videorecorded interview was conducted. Using videoclips to elicit discussion (1), an in-depth interview(2) was used to explore the physiotherapists practice. Using $\mathrm{NVIVO}^{\mathrm{TM}}$, the audio transcripts and video recordings were reviewed and a thematic analysis approach was applied.

ANALYSES: First, the BCT of the video samples and key words used by the physiotherapist were coded. This was followed by repeated listening to the interview, viewing the films, considering the data from the previous study and discussing the findings. Through this approach other codes emerged (41 codes in total). In the next step of analysis, connections were made between the codes and gradually, the story of the physiotherapists approach emerged. Relationships among ideas were turned into a model and another return to the data with the model revealed additional themes. The perspective gained through the analysis of the video recordings from the previous study provided a thick description of the approach. This understanding was reflected upon in discussions with colleagues regarding the cognitive-behavioural approach used in physiotherapy.

RESULTS: There are three elements to the approach that the physiotherapist described: gaining the members trust through building rapport, establishing a need and finding solutions. These elements are related and build upon each other. This approach is applied in a clinical situation that provides ongoing support to members by offering access to physiotherapy services outside the program and, in particular after discharge. The approach, described from the perspective of the physiotherapist, focuses on the interaction between the physiotherapist and members (or the whole group). The main objective of the program is individual transformation, towards an empowered, knowledgeable self-manager. To achieve this, the physiotherapist uses a combination of behavioural change techniques to enhance insight, stimulate decision-making and create effective solutions. The six-week program is carried out in a supportive group environment where there is time taken when necessary to interact one-on-one with the member. Trust established is not just with the physiotherapist, but with the others in the class. The intent of this approach is to engage each member and nudge them along the continuum of change ... so that by the end they will have a better understanding of what they need to do, feel that they can do it (self-efficacy) and be committed to sticking with their program.

CONCLUSIONS: Although the approach described can stand alone, it became clear that it was consistent with other described psychological approaches; the MMPSM (3), Motivational Interviewing (4) and health behaviour change (5) interventions. Physiotherapists use cognitive behavioural strategies in CLBP rehabilitation. The evidence is growing, but we are challenged by the paradigm shift from the biomedical model of practice to the biopsychosocial model in practicing clinicians.(6) The results of this study demonstrate that some of the relatively easy elements that Foster and Delitto(6) have suggested to embed into current practice (e.g., effective reassurance, positive reinforcement, reducing fear of movement), and also some aspects that they suggest require additional training (e.g., eliciting and addressing unhelpful cognitions), are readily available to practicing physiotherapists.

\section{REFERENCES:}

1. Raingruber B. Video-cued narrative reflection: a research approach for articulating tacit, relational and embodied understandings. Qualitative Health Research 2003;13:1155-69.

2. Henninck M, Hutter I, Bailey A. Qualitative Research Methods. SAGE Publications Ltd.; 2011.

3. Jensen M, Nielson W, Kerns R. Toward the development of a motivational model of pain self-management. The Journal of Pain 2003;4:477-92.

4. Rollnick S, Miller W, Butler C. Motivational Interviewing in health care: helping patients change behaviour. New York: 2008.

5. Mason P, Butler C. Health Behavior Change: a guide for practitioners. 2nd ed. Edinburgh: Churchill Livingstone Elsevier; 2010.

6. Foster N, Delitto A. Embedding psychosocial perspectives within clinical management of low back pain: integration of psychosocially informed management principles into physical therapist practice-challenges and opportunities. Phys Ther 2011;91:790-803.

\section{G \\ PAIN IN THE UNDERGRADUATE CURRICULUM: THE DOOR OPENS}

Patricia K Morley-Forster ${ }^{1}$; Eldon Loh'; Gregory McCarthy ${ }^{3}$; Geoffrey Bellingham ${ }^{1}$; David Walton ${ }^{4}$; George Kim ${ }^{5}$; Christopher Brymer6; Dwight E Moulin ${ }^{7}$

${ }^{1}$ Department of Anesthesiology and Perioperative Medicine; ${ }^{2}$ Department of Physical Medicine and Rehabilitation; ${ }^{3}$ Department of Psychiatry; ${ }^{4}$ Department of Physical Therapy; ${ }^{5}$ Department of Family Medicine; ${ }^{6}$ Department of Internal Medicine; ${ }^{7}$ Department of Clinical Neurological Sciences, University of Western Ontario, London, Ontario

AIM: Despite the high prevalence of chronic pain, undergraduate medical instruction on pain management is minimal $(1,2)$. There have been efforts to improve curricula but no standardization exists(3). Mezei et al assessed the pain curricula in North American Medical Schools using the Association of American Medical Colleges (AAMC) Curr MIT, a centralized repository of curricula, and achieved consensus on major topics that should be covered (4).

Following a Needs Assessment of all incoming residents in 2011, our institution's Curriculum Committee mandated an urgent increase in hours devoted to pain management. This report describes the course development.

METHODS: Since 1999, a 12 hour fourth-year selective has been offered by a 7 -member interdisciplinary team at The University of Western Ontario.In spring, 2012, the course leader was asked to increase teaching hours to 18 in 2013, and to 24 in 2014.Additional changes requested were to utilize a case-based approach, to include community physicians and to address pain in the elderly and in the setting of substance abuse.

RESULTS: New instructors were added to create a 13- member team including Family Medicine, specialists, and Allied Health. Topics added were pain assessment , and treatment of patients in primary care, palliative care, geriatric and addicted/ at-risk populations. Cases were drafted by individual instructors then discussed in the group. This revised curriculum will be taught in spring, 2013. Planned additions for 2014 are Pediatric Pain and Medicolegal Aspects of Chronic Pain consistent with the proposal of Mezei et al.

CONCLUSIONS: Health educators will face increasing requests to provide pain curricula. Two specific recommendations are to recruit community and academic faculty willing to work as a team, and to be guided by published curricula.

REFERENCES:

1. Watt-Watson J, et al, 2009 
2. Mezei L, et al, 2011

3. Hunter J, et al, 2008

4. Murinson BB, et al, 2011

\section{$11 \mathrm{H}$}

\section{A MULTIDIMENSIONAL KNOWLEDGE TRANSLATION STRATEGY TO IMPROVE PEDIATRIC PAIN PROCESSES AND OUTCOMES IN 8 PEDIATRIC HOSPITALS IN CANADA}

Bonnie Stevens ${ }^{1}$; Janet Yamada ${ }^{1}$; Jennifer Stinson ${ }^{1}$;

Carole A Estabrooks ${ }^{2}$; Fiona Campbell ${ }^{1}$; Shannon D Scott ${ }^{2}$; Greta Cummings ${ }^{2}$

${ }^{1}$ Hospital for Sick Children and University of Toronto, Toronto, Ontario; ${ }^{2}$ University of Alberta, Edmonton, Alberta

AIM: To describe an innovative, multidimensional knowledge translation (KT) approach, Evidence-based Practice for Improving Quality (EPIQ), and its effect on pediatric pain processes and outcomes in 8 pediatric hospitals in Canada.

METHODS: The EPIQ strategy was developed and effectively implemented to reduce nosocomial infection and bronchopulmonary dysplasia in Neonatal Intensive Care Units (Lee, et al. 2009). The CIHR Team in Children's Pain (Stevens et al., 2006-2011) adapted EPIQ and evaluated the strategy in terms of pediatric pain processes (assessment and management) and outcomes (pain intensity) in 8 pediatric hospitals in Canada, over an 18 month period. 16 units implemented the EPIQ strategy and 16 units continued with standard care. The EPIQ strategy is guided by the Promoting Action on Research Implementation in Health Sciences (PARiHS) framework and rooted in continuous quality improvement (CQI) methods.

RESULTS: The proportion of patients who had pain assessed $(\mathrm{p}<0.002)$, using validated assessment tools, significantly increased in the units that implemented EPIQ compared to standard care units $(\mathrm{p}<0.001)$. The proportion of analgesics administered for procedures increased in both groups, but significantly more in the EPIQ group $(p=0.03)$. The use of physical pain management strategies also significantly increased over time in the EPIQ group $(p=0.02)$. EPIQ was effective in reducing children's pain intensity scores $(\mathrm{p}=0.05)$ during painful procedures in the intervention units.

CONCLUSIONS: Further research into the specific KT strategies used to achieve these outcomes is warranted. As well, replicating the EPIQ strategy and examining similar outcomes in other types of care settings and other clinical practices and populations is recommended.

FOOTNOTES/REFERENCES:

1. Lee SK, Aziz K, Singhal N, et al. Improving the quality of care for infants: A cluster randomized controlled trial. CMAJ 2009;181:469-76.

2. Kitson AL, Rycroft-Malone J, Harvey G, et al. Evaluating the successful implementation of evidence into practice using the PARiHS framework: theoretical and practical challenges. Implemen Sci 2008;3(1).

\section{SESSION $202-11: 00$ AM}

\section{2}

MOBILE (M-HEALTH) AND WEB-BASED TECHNOLOGY TO SHAPE THE FUTURE OF PAIN SELF-MANAGEMENT FOR ADOLESCENTS AND YOUNG ADULTS WITH CHRONIC AND LIFE-LIMITING CONDITIONS

Chair: Susan M Tupper

Speakers: Susan M Tupper; Chitra Lalloo; Lindsay Jibb

WORKSHOP OBJECTIVE:

The overall aim of this session is to describe novel information and communication technologies (Smartphone and Internet-based) to improve the self-management of pain for adolescents and young adults. Dr. Tupper will provide an overview of the advantages and disadvantages associated with these technologies and outline the stages in their development and evaluation. Ms. Lalloo and Jibb will discuss two real-world examples of pain self-management tools for young people living with painful chronic and life-threatening health conditions. Ms. Lalloo will describe the development of iCanCope with Pain, an integrated Smartphone app and website for adolescents and young adults with chronic pain. Ms. Jibb will outline the process of developing a rule-based mobile clinical decision support system for children and adolescents living with persistent cancer pain.

Learning Objectives:

1. To understand the potential benefits of mobile and web-based technology to the field of pain self-management.

2. To understand the theoretical and methodological underpinnings in the development and evaluation of novel mobile and web-based pain selfmanagement tools with a particular focus on end-user involvement.

3. To recognize the challenges associated with the design and implementation of these new technologies and discuss the future of this field.

\section{A}

APPLICATION OF M-HEALTH AND E-HEALTH TECHNOLOGIES TO IMPROVING THE MANAGEMENT OF PAIN IN ADOLESCENTS AND YOUNG ADULTS

\section{Susan M Tupper}

University of Saskatchewan, Saskatoon, Saskatchewan

With emerging interactive information and communication technologies (ICTs), new media for the delivery of health interventions are now available. The use of ICTs in the assessment and management of pain is especially relevant for adolescents and young adults. First, the early adoption of effective disease management strategies by these young patients could help to prevent future costly pain-related disability (physical and psychological morbidity). Second, ICT-based therapies are likely to be readily accepted and adopted by younger individuals ( 12 to 25 year olds) since they represent one of the most active and fastest growing users of this technology. Thirdly, using ICT-based interventions may dramatically reduce funding and temporal constraints on health care delivery. Further, for many young patients living in rural and particularly remote areas, ICT-based pain management tools may be the only treatment available to meet their needs on an ongoing basis. Dr. Tupper will provide an overview of the theoretical foundations and empirical evidence of ICT-based pain management tools, the process of developing and evaluating these technologies, as well as a discussion of associated advantages and disadvantages.

\section{B}

"THERE'S A PAIN APP FOR THAT": PROMOTING PAIN SELF-MANAGEMENT AMONG ADOLESCENTS AND YOUNG ADULTS

Chitra Lalloo

McMaster University, Hamilton, Ontario

BACKGROUND: Adolescents and young adults with chronic pain (AYA; aged $15-25$ years) are expected to assume increasing responsibility for managing their pain due to their growing independence. However, the vast majority of AYA never receive comprehensive education about chronic pain nor coping skills-training to promote disease self-management in order to decrease their physical and emotional symptoms and enhance health-related quality of life. The use of Smartphone and web-based technologies to deliver tailored self-management training may help to optimize and solidify positive health behaviours and prevent pain-related disability within this at-risk group.

METHODS: Qualitative Needs Assessment. Focus groups were conducted with adolescents $(n=16)$, young adults $(n=17)$, and their healthcare providers (HCPs) $(\mathrm{n}=17)$. Individual adolescent interviews were also conducted $(\mathrm{n}=7)$ to better understand AYA information needs.

Scoping Review. The functionality of existing Smartphone apps (iPhone/ Android) related to pain self-management was also reviewed in order to identify gaps in the field.

RESULTS: Qualitative interviews indicated that an integrated Smartphone and web-based self-management program ("iCanCope with Pain') could address the unique developmental needs of AYA by providing improved access to disease information, strategies to manage symptoms, and social support. The scoping review revealed that currently available apps lack: (a) HCP involvement, (b) integrated, personalized features, and (c) foundation in behavioral theory.

CONCLUSIONS: The "iCanCope with Pain" integrated app and website will have four theory-based functionalities: (i) self-monitoring via pain diary, (ii) smart goal-setting related to improving pain and functioning, 
(iii) personalized self-management instruction and skills rehearsal, and (iv) peer-based social support.

\section{$12 \mathrm{C}$ \\ DEVELOPING A SMARTPHONE-BASED PAIN MANAGEMENT TOOL FOR ADOLESCENTS WITH CANCER: ESTABLISHMENT OF STANDARDIZED TREATMENT ALGORITHMS \\ Lindsay Jibb \\ University of Toronto, Toronto, Ontario}

INTRODUCTION: Pain is one of the most common and distressing symptoms reported by adolescents with cancer. Despite advancements in the amount and quality of pain management research, cancer pain in adolescents continues to be poorly managed. A novel strategy to improve pain management in this group involves the use of mobile-health (m-Health) technologies. Algorithms informing specific pain management strategies may improve the self-management of pain and can be conveniently accessed during symptom episodes if delivered using Smartphone technology.

METHODS: A systematic literature review was undertaken to understand the current body of knowledge pertaining to paediatric cancer pain management. Results of this review were disseminated to experts in pain, oncology and $\mathrm{m}$-Health design as well as consumers. These experts and consumers then participated in a 2-day consensus conference to build standardized algorithms (which will provide self-management advice dependant on pain assessments).

RESULTS: The systematic literature review established the current state of research related to physical, psychological and pharmaceutical means for managing paediatric cancer pain. The 2-day consensus conference established rules to inform pain management decisions made by adolescents with cancer.

CONCLUSIONS: Next steps will involve development of the Smartphone interface to deliver management advice to adolescents as well as usability and feasibility testing of the system with adolescents and healthcare providers. Successful development of this Smartphone-based pain management tool has the potential to improve pain management for adolescents with cancer, minimize barriers to optimal symptom treatment, and enhance interaction with healthcare providers to ultimately improve quality of life.

\section{SESSION 203 - 11:00 AM}

\section{3}

\section{THE INFLUENCE OF PAIN ON REWARD PROCESSING AND} POTENTIAL CLINICAL IMPLICATIONS

\section{Chair: Petra Schweinhardt}

Speakers: Petra Schweinhardt; Catherine Cahill WORKSHOP OBJECTIVE:

Avoiding pain and seeking reward are two fundamental motivations for an organism. It is therefore not surprising that pain and reward interact and influence each other. In this workshop, it will be discussed how pain influences reward processing and, on the flip side, how reward influences pain processing. We will look at the interaction between acute pain and reward processing in healthy individuals with respect to behavior and brain processing. Further, alterations of reward processing circuitry in chronic pain states will be demonstrated and potential mechanisms underlying such alterations will be discussed. Potential clinical implications of pain-influences on reward processing and altered reward circuitry in chronic pain will be addressed.

Learning Objectives:

1. Understand the reciprocal influences that pain and reward have on each other.

2. Learn about effects of persistent pain on reward processing circuitry in the brain.

3. Appreciate the clinical implications of altered reward processing circuitry in chronic pain patients.

\section{A}

\section{THE PAIN-REWARD CONNECTION: EVIDENCE FROM HUMAN VOLUNTEERS}

\section{Petra Schweinhardt}

McGill University, Montreal, Quebec

In this presentation, it will be discussed how acute experimental pain in healthy volunteers interacts with reward processing. Specifically, data on the influence of pain on distinct aspects of reward, including the motivation to obtain reward and the pleasure associated with receiving reward, will be presented. The effects of reward and punishment on pain processing will be discussed in terms of behavior and brain processing. Further, the motivational conflict between avoiding pain and seeking reward will be examined via behavioral and brain imaging data, and how the resolution of this conflict is influenced by the homeostatic state of the subject. Lastly, the role of the neurotransmitter dopamine in the processing of pain and in the motivational conflict between pain avoidance and seeking reward will be discussed.

\section{B}

\section{OPIOID REWARD SWITCHES TO A DOPAMINE- DEPENDENT PROCESS IN CHRONIC PAIN: IMPLICATIONS FOR ACTIVATED GLIA IN THE BRAIN}

\section{Catherine Cahill}

University of California Irvine, Irvine, California, USA

Opioids are among the most potent analgesics available, however, the use of opioids for the treatment of non-cancer chronic pain remains controversial due to the significant side effects associated with long-term opioid use, such as addiction. Chronic pain is known to produce profound structural and functional changes in the nervous system that distinguishes it from acute pain and contributes to the refractory nature of this disease. How these changes affect the rewarding aspects of opioids remain to be studied. We used a mouse model of neuropathic pain. The rewarding properties of morphine were determined using the conditioned place preference paradigm.

Pretreatment with the dopamine antagonist, $\alpha$-flupenthixol, blocked the expression of preference for an analgesic dose of morphine in chronic pain, but not control animals, suggesting morphine reward switches to a dopamine-dependent mechanism in a chronic pain state. Additionally, chronic pain resulted in significant activation of microglial cells in the ventral tegmental area (VTA). This activation was associated with a significant increase in brain derived neurotrophic factor (BDNF) in the VTA. Daily, systemic treatment with the microglial inhibitor, minocycline, significantly reduced glial activation in the chronic pain animals. Interestingly, minocycline treatment also blocked the rewarding properties of morphine in chronic pain but not sham animals, echoing the effect of dopamine antagonism on opioid reward in a chronic pain state.

This study shows that morphine reward switches to a dopamine-dependent mechanism in a chronic pain state, and suggests that this switch may be mediated through a microglial dependent mechanism.

\section{SESSION $204-1: 15$ PM}

\section{4}

\section{MEDITATIVE MOVEMENT (TAI CHI, QIGONG) FOR CHRONIC PAIN}

Chair: Mary E Lynch

Speakers: Philip Peng; Mary E Lynch; Jana Sawynok

WORKSHOP OBJECTIVE:

The aim of this symposium is to consider the use of the term "meditative movement" as reflective of a specific set of exercise modalities, review the literature on the efficacy of Tai Chi and Qigong in fibromyalgia and other chronic pain conditions, present some of the challenges involved in research using these complex interventions, and consider clinical trial designs that will be helpful in further elaborating the potential of these practices for management of chronic pain.

Learning Objectives:

1. Participants will be able to define the term "meditative movement" and appreciate some of the challenges in using terminology relating to these practices through lectures and a demonstration. 
2. Participants will learn of the literature on the efficacy of Tai Chi and Qigong in treating and managing fibromyalgia and other chronic pain conditions.

3. Participants will have the opportunity to discuss the potential role of meditative movement in algorithms for management of fibromyalgia.

\section{$14 \mathrm{~A}$}

\section{TAI CHI FOR FIBROMYALGIA AND CHRONIC PAIN} Philip Peng

Department of Anesthesia and Pain Management, University of Toronto, Toronto, Ontario

Tai Chi is an ancient martial and health art that was developed over a few hundred years ago. It involves gentle flowing circular movement of the upper limbs, constant weight shifting of lower limbs, meditation, breathing, moving of qi (the internal energy in Chinese belief) and various techniques to train mind-body control. It is a mild to moderate aerobic exercise. Because the movements are slow and gentle, an individual with chronic illness and pain can pace according to their physical fitness. The constant shifting of body weight helps to improve the balance, minimize the risk of fall and maintain bone mineral density in post-menopausal women. Tai Chi helps the individual to focus and improve their mind-body control. The role of higher centers in pain modulation is well supported by an abundance of literature.

Although a volume of literature has been published examining the role of Tai Chi in pain management, the literature is limited in its study design. A recent systematic review showed high quality research supporting the beneficial role of Tai Chi in fibromyalgia, osteoarthritis and low back pain. More high quality research is required in the future.

\section{B}

\section{A RANDOMIZED CONTROLLED TRIAL OF QIGONG FOR FIBROMYALGIA: THE HALIFAX EXPERIENCE}

Mary E Lynch

Department of Anesthesiology, Psychiatry, Pharmacology,

Dalhousie University, Halifax, Nova Scotia

Several years ago, following publication of a trial from Sweden that reported sustained benefits of qigong for fibromyalgia, we undertook a pilot trial to examine potential efficacy of a form of qigong that was available locally in Halifax (CFQ). Results were promising, so we undertook a randomized controlled trial $(\mathrm{N}=100)$ in which $\mathrm{CFQ}$ practice was compared to wait-list subjects who continued with their usual care; at the end of the wait list interval, these individuals also received CFQ training. Training consisted of 3 half-days of instruction from qualified CFQ personnel and weekly review sessions for 8 weeks, and trial participation required daily practice of 45 mins for 6 months. Pain (NRS-PI), impact (FIQ), sleep (PSQI), and physical and mental function (SF-36) were assessed at baseline and 8 weeks, and then at 4 and 6 months. Compared to the wait list, CFQ practice led to significant reductions in pain, impact, sleep disturbance, and improvements in physical and mental function in both training groups (immediate, delayed) at 8 weeks, and this was sustained at 4 and 6 months. For pain, $38 \%$ to $47 \%$ compared to $9 \%$ to $18 \%$ of wait list subjects attained a pain reduction of $\geq 30 \%$, while for impact, $56 \%$ to $70 \%$ compared to $20 \%$ to $34 \%$ of wait list subject attained a score reduction of $\geq 14 \%$ (meaningful changes). Post-hoc analysis in relation to extent of practice indicated more benefit in those who practiced according to protocol. These promising results indicate that qigong may be a useful self-care practice for those with fibromyalgia, and are consistent with our clinical experience with chronic pain patients who practice qigong consistently for 45 to 60 minutes per day. The results of the study, and applications from clinical experience, will be presented.

\section{$14 \mathrm{C}$}

\section{THE PROMISE AND CHALLENGES OF RESEARCH IN TAI CHI AND QIGONG: METHODOLOGICAL ISSUES \\ Jana Sawynok}

Department of Pharmacology, Dalhousie University, Halifax, Nova

Scotia

There is currently an emerging literature on the benefits of tai chi and qigong in a variety of chronic health conditions. In 2009, these practices were characterized as "meditative movement", and this has been very helpful for understanding what they entail and for considering their health potential. These practices have a long history, occur in many forms, and come from traditions in which terminology does not match with conventional biomedical models, so the introduction of this phrase provides two core domains that can be examined independently or together. There are now two recently published randomized controlled trials which demonstrate the efficacy of tai chi (Wang et al, New Eng J Med, 2012) and qigong (Lynch et al, Arth Res Ther, 2012) in fibromyalgia, and these indicate sustained benefits to 6 months over several core domains (pain, impact, sleep, physical and mental wellbeing). Case studies reveal that when individuals engage in diligent practice over sustained intervals, health benefits extending beyond this core symptom relief can occur. Pragmatic issues of minimal practice requirements in order to see benefits ("threshold effect" or minimal dose concept) and relationship to extent of practice ("practice-response relationship") are being addressed. The challenge of what is a suitable control group for such practices, the types of comparative studies that will be helpful in further exploring these modalities, and the language and constructs that are needed to further explore this area will be considered.

\section{SESSION $205-1: 15$ PM}

HOT TOPICS

\section{A}

\section{AGE-RELATED PATTERNS IN SENSATION THRESHOLD AND PAIN THRESHOLD}

N Ciccone ${ }^{1,2}$; A Easson ${ }^{3}$; M Li ${ }^{1}$; G Rodin ${ }^{1}$; G Koren ${ }^{4}$; V Chan ${ }^{5}$; L Gagliese ${ }^{1,2,5}$

${ }^{1}$ Psychosocial Oncology and Palliative Care, University Health Network; ${ }^{2}$ York University; ${ }^{3}$ Surgical Oncology, University Health Network; ${ }^{4}$ Motherisk Program, Hospital for Sick Children; ${ }^{5}$ Department of Anesthesia, University Health Network, Toronto, Ontario

AIM: Age differences in sensation threshold (ST) and pain threshold (PT) are inconsistent, with increases, decreases, and no changes reported. Past studies have not considered the impact of biopsychosocial variables, including health status, on this relationship. This study assessed the role of age and biopsychosocial variables in ST and PT.

METHODS: 107 participants ( 53 men; 54 women) scheduled for surgery were recruited from University Health Network, Toronto, Ontario. Preoperative demographic and psychological questionnaires (Pain Catastrophizing Scale, Center for Epidemiologic Studies Depression Scale, State-Trait Anxiety Inventory, Pain Anxiety Symptom Scale, Pain History Questionnaire) were completed. Three quantitative sensory tests were administered at the right ankle: thermal (TSA II Neurosensory Analyzer, Medoc), pressure (Algometer, Somedic), and punctate (Aesthesiometer, Somedic).

RESULTS: Seven models of ST and PT (warm, cool, and punctuate (ascending and descending) ST; hot, cold, pressure PT) were constructed. Age was a predictor of all but cool and punctuate STs. Sex was the only predictor retained in each model, and the strongest predictor in each. Health Status measures (symptomatology, comorbidity, performance status, blood pressure, body mass index, chronic pain, polypharmacy, smoking history) were correlated with age and were predictors of ST or PT. Other predictors included state, trait, and pain anxiety, and pain expectations. CONCLUSIONS: This is the first study to demonstrate that biopsychosocial factors, especially health status indicators, are important predictors of pain and sensation thresholds. These results may help elucidate the relationship between advancing age and pain sensitivity.

\section{B}

NEW RISK FACTORS FOR OPIOID-RELATED DEATHS IN ONTARIANS

Parvaz Madadi ${ }^{1}$; Doris Hildebrandt ${ }^{2}$; Albert Lauwers ${ }^{2}$; Gideon Koren ${ }^{1}$

${ }^{1}$ Hospital for Sick Children; ${ }^{2}$ Office of the Chief Coroner of Ontario, Toronto, Ontario

AIM: To characterize risk factors amongst Ontarians whose cause of death was opioid-related between the years 2006 and 2008. 
METHODS: This was a population-based study of Ontarians between the years 2006 and 2008. All drug-related deaths during this time frame were reviewed at the Office of the Chief Coroner of Ontario, and opioid-related deaths were identified. Medical, toxicology, pathology, and police reports were analyzed.

RESULTS: Out of 2330 drug-related deaths in Ontario, 58\% were attributed either in whole or in part, to opioids $(n=1359)$. Oxycodone was involved in approximately one-third of all opioid-related deaths, and methadone was associated with the highest proportion of accidental deaths. Codeine was disproportionately utilized in suicides. At least $8 \%$ of the entire cohort used opioids that were prescribed for friends and/or family, 19\% inappropriately self-administered opioids (injection, inhalation, chewed oral patch), $3 \%$ were recently released from jail, and $5 \%$ had been switched from one opioid to another near the time of death. Accidental deaths were significantly associated with higher opioid risk scores, enrollment in a methadone maintenance program, cirrhosis, hepatitis, and cocaine use. Suicides were significantly associated with mental illness, previous suicide attempts, chronic pain, and a history of cancer.

CONCLUSIONS: We have identified novel risk factors associated with opioid-related deaths in Ontarians. These findings point to the need for multifaceted prevention strategies based on subpopulations of opioid users.

\section{C}

\section{ELICITATION OF EMPATHY FOR PAIN IN STRANGERS BY} SOCIAL STRESS REDUCTION IN MICE AND HUMANS

Loren Martin ${ }^{1}$; Georgia Hathaway ${ }^{1}$; Sara Mirali'; Kelsey Isbester ${ }^{1}$; Nils Niederstrasser'; Peter Slepian ${ }^{1}$; Zina Trost ${ }^{2}$; Wendy

Sternberg'; Robert Sapolsky ${ }^{4}$; Daniel Levitin ${ }^{3}$; Jeffrey Mogil ${ }^{1}$

${ }^{1}$ McGill University, Montreal, Quebec; ${ }^{2}$ University of North Texas,

Denton, Texas; ${ }^{3}$ Haverford College, Haverford, Pennsylvania;

${ }^{4}$ Stanford University, Stanford, California, USA

AIM: We have previously demonstrated that mice who observe their cagemates in pain are more sensitive to pain themselves - an effect considered to be a form of empathy (Science: 312(5782):1967-70). However, mice who observed an unfamiliar partner (i.e. stranger mouse) in pain did not demonstrate this hyperalgesia. In order to determine whether the lack of hyperalgesia in stranger pairs is due to social stress, we pharmacologically inhibited the synthesis of glucocorticoids in mice and subsequently blocked either glucocorticoid or mineralcorticoid receptors. In an attempt to explore the same phenomenon in humans, we tested whether people are more sensitive to pain when they observe a friend in pain compared with people observing a stranger and whether stress may play a role.

METHODS: Mouse experiments. Mice were habituated for $30 \mathrm{~min}$ to a Plexiglas cylinder in either dyads (cagemates or strangers) or in isolation. At the beginning of the habituation period mice were injected systemically with metyrapone $(50 \mathrm{mg} / \mathrm{kg})$, a glucocorticoid synthesis blocker, mifeprestone $(10 \mathrm{mg} / \mathrm{kg})$, a glucocorticoid receptor blocker or RU-26752 (5 mg/kg), a mineralcorticoid receptor blocker. Following the habituation period mice received a single intraperitoneal injection of $0.9 \%$ acetic acid and the number of abdominal constrictions ("writhing") was recorded in order to assess nociceptive sensitivity. Human experiment. McGill university undergraduate students were recruited and tested in either friend or stranger dyads. Stress reduction was done with pharmacological administration of the cortisol synthesis blocker metyrapone $(750 \mathrm{mg})$ or subjecting pairs of strangers to a social gaming experience (playing the video game Rock Band together for $20 \mathrm{~min}$ ). All participants were required to rate the intensity and unpleasantness of cold water during two counterbalanced 30-s pain trials, one in which they were tested alone and one facing the other participant. All subjects completed the profile of mood states (POMS) and pain catastrophizing scale (PCS) questionnaires before testing and a subset of the interpersonal reactivity index (IRI), following pain testing. Salivary cortisol was also measured throughout the experiment and subjects were given an exit questionnaire to assess the degree of relationship with the other participant. All experiments were approved by the McGill university research ethics board.

RESULTS: In the mouse studies, as previously demonstrated, stranger mice did not display hyperalgesia when both mice were in pain, but pretreatment with either metyrapone or a combination of mifeprestone and RU-26752 revealed an empathy-like hyperalgesia similar to that shown by cagemate pairs. In the human study, the mean difference for intensity and unpleasantness ratings was significantly increased in people who observed their friends in pain compared to people who observed a stranger. The change in the intensity and unpleasantness ratings for strangers was negatively correlated with the anxiety scale of the POMS, indicating that for the stranger condition more anxiety (i.e., social stress) was associated with less hyperalgesia and in some cases analgesia. Further, blocking cortisol synthesis with metyrapone or playing the video game Rock Band revealed a hyperalgesia in stranger dyads that was similar to the friend condition. CONCLUSIONS: Social-stress was associated with decreased pain empathy in stranger mice and blocking either glucocorticoid synthesis or glucocorticoid receptors revealed the empathic effect. Similar findings were obtained in humans; and the effect of pharmacological stress blockade and alleviation is hyperalgesic on self-reported pain ratings in stranger dyads.

\section{D}

PHARMACOKINETICS OF OPIOIDS DURING HEMODIALYSIS: METHADONE VS. HYDROMORPHONE

Ryan Perlman'; ${ }^{1}$ Hili Giladi²; Krista Brecht'; Mark Ware ${ }^{2}$; Terence Hébert ${ }^{3}$; Lawrence Joseph ${ }^{4}$; Yoram Shir ${ }^{2}$ ${ }^{1}$ The Alan Edwards Pain Management Unit, Department of Anesthesiology, McGill University Health Centre (MUHC), Montreal General Hospital; ${ }^{2}$ The Alan Edwards Pain Management Unit, McGill University Health Centre (MUHC), Montreal General Hospital; ${ }^{3}$ Department of Pharmacology and Therapeutics, McGill University; ${ }^{4}$ Department of Clinical Epidemiology and Biostatistics, McGill University Health Centre (MUHC), Royal Victoria Hospital, Montreal, Quebec

AIM: Opioids are commonly prescribed to patients with end-stage kidney disease and chronic pain requiring hemodialysis. Stability of opioid analgesia during dialysis could vary among different opioids. No studies to date have corroborated this clinical observation by directly comparing plasma levels of different opioids during dialysis. We compared changes in the peri-dialysis plasma levels of two pharmacokinetically distinct opioids, methadone and hydromorphone (HM).

METHODS: Fourteen dialysis patients with chronic pain necessitating opioid therapy received either methadone or $\mathrm{HM}$ for at least 2 weeks prior to beginning the study. Blood samples were obtained immediately before, during and after hemodialysis in two separate dialysis sessions, one week apart, and were analyzed for opioid levels. The primary outcome was the change in plasma opioid concentrations during dialysis. Secondary outcomes included pain levels before and after dialysis, opioid-related adverse effects and quality of life.

RESULTS: The mean percent change of methadone plasma levels was $13.3 \pm 8.6 \%$ compared to $60.0 \pm 8.5 \%$ in the HM treatment group, corresponding to a difference of $46.7 \%$ (95\% CI 22.52 to 71.22 ). The mean plasma clearance of methadone was $24.8 \pm 8.7 \mathrm{ml} / \mathrm{min}$ compared to $100.7 \pm 9.5 \mathrm{~mL} / \mathrm{min}$ for HM. The mean difference between the opioid clearance levels was $75.9 \mathrm{~mL} / \mathrm{min}$ ( $95 \%$ CI 46.9 to 99.4 ). There were no differences between the two opioid groups in any of the secondary outcomes. CONCLUSIONS: Methadone plasma levels were significantly more stable during hemodialysis compared to HM. Methadone therapy was not associated with an increased rate of adverse events. If confirmed by larger clinical studies, methadone could be considered as one of the opioids of choice in dialysis patients.

\section{E}

\section{TREATMENT RESISTANCE OF DEPRESSIVE SYMPTOMS TO MULTIDISCIPLINARY REHABILITATION FOR WHIPLASH INJURY: THE ROLE OF PERCEIVED INJUSTICE}

Whitney Scott; Michael JL Sullivan

McGill University, Montreal, Quebec

AIM: Depressive symptoms complicate patients' recovery following musculoskeletal injury. Research suggests that a number of interventions are associated with significant reductions on depressive symptoms following injury. Despite this, a significant proportion of patients do not experience meaningful reductions on depressive symptoms following intervention (Sullivan et al., 2006). Emerging evidence suggests that initial severity of depressive symptoms, pain catastrophizing, self-efficacy, and perceived injustice may contribute to the maintenance of depressive symptoms among patients undergoing unimodal treatment programs. The purpose of 
this study was to examine predictors of depressive symptom treatment resistance among whiplash-injured patients undergoing multidisciplinary rehabilitation.

METHODS: One hundred and three patients with persistent pain following whiplash injury participated in this study. Self-report measures of depressive symptoms, pain intensity, disability, and pain-related psychosocial variables were completed by patients upon commencement and completion of a standardized multidisciplinary rehabilitation program. Analyses examined which pre-treatment variables predicted depressive symptom treatment resistance. Treatment resistance was defined as remaining above a clinical threshold on the measure of depressive symptoms at pre-and post-treatment.

RESULTS: Univariate analyses indicated that pre-treatment scores on depressive symptoms, pain catastrophizing, and perceived injustice were significantly higher among patients whose depressive symptoms were resistant to treatment, as compared to patients who experienced a clinically meaningful reduction on depressive symptoms. A logistic regression analysis revealed that perceived injustice was the only variable that contributed significant unique variance to the prediction of depressive symptom treatment resistance, OR 1.12 (95\% CI 1.01 to 1.26 ); $\mathrm{p}<0.05$.

CONCLUSIONS: The results suggest that perceived injustice is an important psychosocial variable to consider within interventions targeting depressive symptoms among patients with persistent musculoskeletal pain. Potential mediating processes and intervention strategies will be discussed.

\section{$15 \mathrm{~F}$}

\section{CERVICAL RADIOFREQUENCY NEUROTOMY REDUCES PSYCHOLOGICAL DISTRESS AND PAIN CATASTROPHIZATION, BUT NOT POST-TRAUMATIC STRESS IN INDIVIDUALS WITH CHRONIC WAD}

Ashley Smith ${ }^{1}$; Gwendolen Jull ${ }^{1}$; Geoff Schneider ${ }^{2}$; Bevan Frizzell2; R Allen Hooper ${ }^{2}$; Michele Sterling ${ }^{3}$

${ }^{1}$ Spinal Injury, Pain and Health, Division of Physiotherapy, School of Health and Rehabilitation Sciences, University of Queensland, Queensland, Australia; ${ }^{2}$ Faculty of Medicine, University of Calgary, Calgary, Alberta; ${ }^{3}$ Centre for National Research on Disability and Rehabilitation Medicine (CONROD), University of Queensland, Queensland, Australia

AIM: To determine if reducing nociception in the cervical spine (following successful RadioFrequency Neurotomy) significantly reduces the psychological manifestations in chronic WAD (psychological distress, pain catastrophization and severity of post-traumatic stress symptoms).

METHODS: This longitudinal study involved 38 WAD individuals; resistant to improvement with conservative health care; who responded to cervical RFN. Measures were collected at t1 (entry to study), t2 (10 to 12 months later, immediately prior to RFN), t3 (one month following RFN) and t4 (three months following RFN). Pain (VAS), disability (NDI) and quality of life (SF-36) measures were collected. Psychological measurements included the General Health Questionnaire (GHQ-28); Pain Catastrophization Scale (PCS) and the severity index of the Post Traumatic Stress Diagnostic Scale (PDS).

ANALYSIS: One-way ANOVA was performed for pain, disability and quality of life measures. Kruskal-Wallis rank tests were performed for the psychological measures. Alpha was set at $\mathrm{p}<0.05$.

RESULTS: No change in pain, disability or psychological measures occurred prior to receiving cervical RFN. Cervical RFN resulted in immediate (within one month) and sustained improvements in pain $(\mathrm{p}<0.001)$, disability $(p<0.001)$, and quality of life $(p<0.001)$. There was a significant reduction in psychological distress $(\mathrm{GHQ}-28 ; \mathrm{p}<0.001)$ and pain catastrophization $(\mathrm{p}<0.001)$, but not post-traumatic stress $(\mathrm{p}>0.48)$ severity measures.

CONCLUSIONS: Decreasing peripheral nociception via cervical RFN resulted in significant improvement in psychological distress and pain catastrophization. However, despite improved pain and disability levels, and quality of life, individuals demonstrated ongoing post traumatic stress symptoms. Further research is needed to further understand the mechanisms underlying persistent post-traumatic stress symptoms.

\section{SESSION $206-1: 15$ PM}

\section{6}

\section{BACK TO BASICS: AN UPDATE ON THE MECHANISMS AND MANAGEMENT OF LOW BACK PAIN}

\section{Chair: Mark A Ware}

Speakers: Laura Stone; Mark A Ware; Susan M Tupper

WORKSHOP OBJECTIVE:

The main objective of this symposium is to encourage a dialogue on current approaches to understanding and treating low back pain (LBP). Three different perspectives will be presented, from bench to bedside to policy, and we will foster a dialogue among participants about the barriers and facilitators to improving our care of patients with this challenging condition.

Learning Objectives:

1. Session attendees will a) understand current pre-clinical research on sources of nociception that contribute to low back pain, and b) discuss new insights gained into the relationship between low back pain, radicular pain, and disc degeneration.

2. Attendees will explore ways to identify the patient with low back pain at risk of chronicity and will develop a strategy to implement interdisciplinary management of these patients

3. Attendees will be able to describe three programs for the evaluation of patients presenting to primary care with low back pain, and reflect on the methods to measure their outcomes.

\section{A}

\section{BASIC SCIENCE MECHANISMS OF LOW BACK PAIN} Laura Stone

Dentistry, Alan Edwards Centre for Research on Pain, McGill University, Montreal, Quebec

While disc degeneration is associated with increased risk of chronic low back pain (LBP) in humans, the relationship between disc pathology and the behavioural signs of LBP remain unclear. An animal model of progressive, age-dependent degenerative disc disease (DDD) associated with behavioural indices of low back pain has been developed to address these questions. This session will review our understanding of the basic mechanisms underlying LBP with new insights into the relationship between the brain, low back pain, radicular pain, disc degeneration, along with recent work exploring anatomical and functional changes in the brain associated with low back pain and the ability of effective treatment to reverse these pathologies. New insights into the relationship between the development of DDD and the emergence of behavioral signs of low back and radicular pain will be discussed.

\section{B}

\section{SHADES OF PAIN: THE CLINICAL PICTURE OF LOW BACK PAIN}

Mark A Ware

Anesthesia and Family Medicine, Alan Edwards Pain Management Unit, McGill University Health Centre, Montreal, Quebec

It is sad but true that low back pain remains one of the most common reasons for patients to be seen in primary care and tertiary pain clinics, and represents a huge burden on the public health system both directly (e.g. clinic visits, procedures, imaging) and indirectly (e.g. lost work productivity, absenteeism). A wide range of methods to classify and define back pain have been proposed, based on duration, cause, radiological findings, interventions, and clinical presentation. This presentation will review the most well accepted approaches to the diagnosis and management of low back pain, and will highlight recent approaches in Quebec to prevent the chronicity of low back pain using early interdisciplinary interventions. 


\section{$16 \mathrm{C}$}

\section{UTILIZING INTERPROFESSIONAL RESOURCES TO STREAMLINE MANAGEMENT OF BACK PAIN}

\section{Susan M Tupper}

School of Physical Therapy, University of Saskatchewan, Saskatoon, Saskatchewan

Wait times have been identified as a significant barrier to accessing quality health care, and wait for orthopedic surgery consult is one of the longest of all specialists (approximately 17 weeks). Delay in access to appropriate care can lead to patient distress related to uncertainty about diagnosis and prognosis, reduced quality of life and reduced physical function. Serious spinal pathologies are found in only a very small proportion (approximately 1\%) of patients presenting to primary care with low back pain (LBP), and the vast majority of patients are not surgical candidates. However, patients with LBP continue to represent a large proportion of referrals to orthopedic specialists. The Saskatchewan Spine Pathway Clinics (two publicly funded clinics) and the Wall Street Spinal Assessment Service (a private clinic) use two distinct approaches that utilize physiotherapists to screen surgical referrals for LBP in Saskatchewan. These programs will be described and outcomes on surgical wait times and patient reported outcomes (satisfaction, quality of life, physical function) will be presented. Utilizing physiotherapists to triage patients referred to orthopedic specialist for LBP can reduce the number of non-surgical cases and improve timely access to appropriate care.

\section{SESSION $207-3: 45$ PM}

\section{7}

\section{PAIN IN EMERGENCY MEDICINE - THE WHOLE STORY}

Chair: James Ducharme

Speakers: James Ducharme; Sean Moore; Sylvie Lemay

WORKSHOP OBJECTIVE:

1) Describe the breadth of pain pathology seen in the $E D$

2) Define ways to distinguish aberrant drug related behaviours as a result of pseudoaddiction from those of addiction in the acute setting

3) Establish strategies to mange acute pain in patients who may be or are addicts

4) Discuss both phramacological and non-pharmacological methods of controlling pain in the ED

Learning Objectives:

1. Increase understanding of patients in pain in the ED, including understanding the role the ED plays for patients with chronic pain

2. To best define pain management strategies in the ED - what works, what does not work and what the ED cannot do.

3. To identify optimal approaches for managing pain in the addict

\section{A}

\section{THE BREADTH OF PAIN IN THE EMERGENCY DEPARTMENT}

\section{James Ducharme}

CPS, McMaster University, Hamilton, Ontario

Most professionals view pain care in an ED limited to that associated with acute illness or injuries. This is not the case, for up to $20 \%$ of all ED patients are presenting with chronic pain as the source of their primary complaint.This presentation will review the spectrum of pain seen in an emergency department

\section{B}

\section{PAIN, ADDICTION AND DRUG SEEKING IN THE EMERGENCY DEPARTMENT}

\section{Sean Moore}

University of Ottawa, Ottawa, Ontario

Diversion of prescription opioids has become a hot topic, with it being most evident in emergency departments. Evaluating patients and distinguishing pain and pseudoaddiction from addiction and diversion is at best difficult. This presentation will review how to objectively do so, while ensuring pain is properly managed

\section{C}

\section{PHARMACOLOGICAL AND NON-PHARMACOLOGICAL METHODS TO RELIEVE PAIN}

Sylvie Lemay

Université de Montréal, Montreal, Quebec

Managing acute pain and the emotional loss of control associated with it is never solved by just giving a medication. Appropriate medication choice combined with education, the return of control to the patient and the use of non-pharmacological methods are as important in helping the patient. This presentation will provide the attendee practical examples on how to best manage pain in the ED.

SESSION 208 - 3:45 PM

\section{8}

\section{NEW STANDARDS FOR DEVELOPMENT OF CANADIAN PAIN SOCIETY CLINICAL PRACTICE GUIDELINES}

Chair: Mary-Ann Fitzcharles

McGill University Health Centre, Division of Rheumatology and Alan Edwards Pain Management Unit, Montreal, Quebec Speakers: Michael McGillion; Peter A Ste-Marie; John X Pereira WORKSHOP OBJECTIVE:

To present the new Canadian Pain Society (CPS) policy and procedures for development of high-quality clinical practice guidelines for the management of various painful conditions. CPS 2012 Guidelines will be presented from the perspective of the new requirements for guideline development and implementation. This workshop will be of key importance to future CPS guideline developers.

Learning Objectives:

1. To understand the new policy and procedures providing direction to the development and evaluation of new CPS evidence-based clinical practice guidelines.

2. To understand how to appraise the internal and external validity of practice recommendations using the AGREE II framework.

3. To examine, in detail, the process of developing the new 2012 Canadian Fibromyalgia Guidelines. To understand key recommendations for the treatment of fibromyalgia and recognize barriers inherent in effective implementation.

\section{A}

\section{THE NEW CPS DEVELOPMENT AND REVIEW PROCESS}

\section{Michael McGillion}

Lawrence S Bloomberg Faculty of Nursing, University of Toronto, Toronto, Ontario

Multiple factors influence the confidence we can place in recommendations put forth in clinical practice guidelines. Judgments about the strength of each recommendation require practiced consideration of the clinical context of intervention delivery, potential benefits versus harms, and the methodological quality of the available evidence. The complexity of practice guideline development has led to widespread usage of multiple systems for evaluating the strength of clinical practice recommendations. Limitations of most systems include: a) reliance on implicit definitions of methodological quality, b) inconsistency in the depth of methodological evaluation and reporting of findings across clinical outcomes, and c) insufficient evaluation of clinical benefits versus harms. For these reasons, the CPS has developed new policy and procedures for the development and evaluation of clinical practice guidelines based on standards put forth by the Appraisal of Guidelines for Research and Evaluation (AGREE) - II instrument.

Examples from the development of the 2012 Joint Canadian Cardiovascular Society (CCS) - Canadian Pain Society (CPS) Guidelines for the Management of Refractory Angina, as well as hands-on exercises, will be used to explain the new CPS process for guideline developers, with attention to appraising guidelines according to the following six domains of AGREE II, including: scope and purpose, stakeholder involvement, rigour of development, clarity of presentation, applicability, and editorial independence. 
18B

DEVELOPING THE 2012 FIBROMYALGIA GUIDELINES: LESSONS LEARNED

\section{Peter A Ste-Marie}

Faculty of Law, Université de Montréal, Montreal, Quebec

The development of de novo national guidelines for clinical care of various conditions can be a daunting task. The methodology used to develop new Canadian guidelines for fibromyalgia will be explained and comment will be made on challenges when following this rigorous process. Specific areas to be addressed will include the access to full scope of literature, costs related to guideline development, time commitment for participants and contribution and participation of a large multidisciplinary committee. The process followed by the FM guidelines working group was guided by the Appraisal of Guidelines for Research and Evaluation II recommendations (AGREE II), and evidence was evaluated using the Oxford Centre for Evidence-Based Medicine Evaluation Table. The main message of the guidelines which addresses the diagnosis, management and clinical trajectory of patients with FM in Canada will be discussed. Particular comment will be made on recommendations that were contentious when reviewed by the national advisory panel and therefore required revision. Key learning points from attaining final team consensus, crafting the final document, and creating plans for guideline dissemination will also be discussed.

\section{$18 \mathrm{C}$}

IMPLEMENTING THE 2012 FIBROMYALGIA GUIDELINES: KEY RECOMMENDATIONS, CHALLENGES, AND OPPORTUNITIES

John X Pereira

Department of Family Medicine, Faculty of Medicine, University of Calgary, Calgary, Alberta

Development and publication of guidelines must be seen as the first step in effecting any change in clinical care. Although guidelines are developed with the best intentions and supported by input usually from a multidisciplinary working group, the practical implementation and success of the recommendation process is dependent upon efficacy of dissemination and the acceptance by the healthcare community. Effecting a paradigm change in clinical concepts such as has been proposed by the 2012 Canadian Fibromyalgia Guidelines will require a change from the negative perceptions associated with this condition from both the healthcare community and patient perspectives. Lessons learned from pharmaceutical marketing strategies have taught us that simply targeting healthcare professionals, without addressing the wider patient community will not be successful. Fibromyalgia, a condition that has wide reaching socioeconomic and societal effects, should now be accepted as a valid medical syndrome, not requiring excessive healthcare utilization and with emphasis on maintenance of function. Key recommendations from the new FM guidelines will be reviewed, as well as key challenges and opportunities with respect to guideline implementation.

\section{SESSION $209-3: 45$ PM}

\section{9}

\section{ASSESSMENT OF CANCER PAIN ACROSS THE LIFESPAN:} EVIDENCE, CHALLENGES AND FUTURE DIRECTIONS

\section{Chair: Lucia Gagliese}

Speakers: Jennifer Stinson; Lynn Gauthier; Lucia Gagliese WORKSHOP OBJECTIVE:

The overall aim of this symposium is to explore the challenges of assessing cancer pain in people of different ages (from children to older adults) and different stages of disease (from early to end of life).

Learning Objectives:

1. Describe self-report pain assessment tools for use across the adult lifespan

2. Discuss innovative methods for obtaining self-reports in cancer pain including pain apps

3. Explore barriers to effective pain assessment across the lifespan
19A

\section{PAIN ASSESSMENT IN CHILDREN AND YOUTH WITH CANCER: CHALLENGES AND NOVEL APPROACHES Jennifer Stinson}

Hospital for Sick Children; Lawrence S Bloomberg Faculty of Nursing, University of Toronto, Toronto, Ontario

Pain is one of the most common and distressing symptoms reported by children and youth with cancer. Despite advancements in the amount and quality of pain management research, pain in adolescents with cancer continues to be poorly managed. While there are tools to measure pain and other symptoms in children with cancer they are not routinely used in everyday clinical practice due to participant burden and lack of clinical utility. Moreover they rely on recall. Novel approaches using mobilehealth applications (or Apps) are ideally suited for this population as youth are early adopters of this technology. Dr. Stinson will present data on the reliability and validity including responsiveness of the PainSquad Cancer Pain App. She will also discuss the prevalence and impact of cancer pain in this population. Finally she will discuss future avenues for research including the development of a clinical decision-making tool to promote the management of pain in community settings.

\section{B}

\section{SELF-REPORT TOOLS FOR CANCER PAIN ASSESSMENT ACROSS THE ADULT LIFESPAN}

Lynn Gauthier

School of Kinesiology and Health Science, York University; Psychosocial Oncology and Palliative Care, University Health Network, Toronto, Ontario

Pain is highly prevalent in adults with cancer and is associated with impairments in multiple domains of wellbeing. With the aging population, there will be increasing numbers of older people who will require treatment for cancer pain. Unfortunately, cancer pain in older people remains poorly understood and undertreated. Proper palliation of pain depends on assessment methodologies that are valid and reliable for both younger and older people with cancer. In this presentation, Ms. Gauthier will give an overview of cancer pain assessment across the adult lifespan, highlight the limitations of certain assessment methodologies and gaps in our knowledge, and discuss unique considerations for pain assessment in older, cognitively intact patients. She will present findings from a recently completed study validating a multidimensional measure of pain qualities, the Short-Form McGill Pain Questionnaire-2, for younger and older cancer patients. She will also discuss future research directions designed to improve our understanding of the biopsychosocial model of cancer pain in younger and older people.

\section{C}

\section{PAIN IN OLDER CANCER PATIENTS WITH DELIRIUM AT THE END OF LIFE: TOWARDS A STANDARDIZED ASSESSMENT PROTOCOL}

Lucia Gagliese

School of Kinesiology and Health Science, York University; Psychosocial Oncology and Palliative Care, Anesthesia \& Pain Management, Ontario Cancer Institute, University Health Network, Toronto, Ontario

Both pain and delirium are highly prevalent among older cancer patients at the end of life. However, little is known about the mechanisms underlying their probable inter-relatedness. Importantly, to date, a validated pain assessment protocol for this vulnerable group of patients is not available, limiting research and clinical assessment. In this presentation, Dr. Gagliese will present data on pain and delirium among older people in an inpatient palliative care unit, with special focus on the prevalence of pain at the very end of life, its correlates and the strategies employed by healthcare workers when assessing pain. This will be complemented by qualitative data describing healthcare workers' experiences and beliefs about pain in this group of patients. The discussion will focus on the many challenges and obstacles encountered by healthcare workers' when assessing pain in older cancer patients with delirium at the end of life and will consider future research and clinical directions to overcome these obstacles. 
FRIDAY MAY 10, 2013

SYMPOSIA - 8:00 AM

20

Chair: Dwight Moulin

Departments of Clinical Neuro Sciences/Oncology, Earl Russell

Chair Pain Medicine, University of Western Ontario, London,

Ontario

\section{A}

CHANGING THE PARADIGM FROM TREATMENT TO PREVENTION: STRATEGIES FOR REDUCING THE BURDEN OF HERPES ZOSTER AND POST HERPETIC NEURALGIA

Speakers: Marla Shapiro ${ }^{1}$; Kathryn Slayter ${ }^{2}$

${ }^{1}$ Family Physician, Associate Professor, Department of Family and Community Medicine, University of Toronto, Toronto, Ontario; Medical Consultant, CTV and Canada AM; ${ }^{2}$ Clinical Pharmacy Specialist, Division of Infectious Diseases, Department of Medicine; Adjunct Assistant Professor, Faculties of Medicine and Health Professions, Clinical Scientist, Canadian Center for Vaccinology, Dalhousie University, Halifax, Nova Scotia

Learning Objectives:

By the end of the session, participants will be able to:

1. Recognize the epidemiology and burden of disease associated with $\mathrm{HZ}$ and PHN.

2. Describe the pathophysiology and clinical manifestations of $\mathrm{HZ}$ and $\mathrm{PHN}$.

3. Assess the recent data and potential benefits of immunization to prevent $\mathrm{HZ}$ and PHN and to reduce pain severity and duration of $\mathrm{HZ}$.

Herpes zoster (HZ), commonly called shingles, is a distinctive syndrome caused by reactivation of varicella zoster virus (VZV). This reactivation occurs when immunity to VZV declines because of aging or immunosuppression. Herpes zoster can occur at any age but most commonly affects the elderly population. Postherpetic neuralgia ( $\mathrm{PHN}$ ), defined as pain persisting more than 3 months after the rash has healed, is a debilitating and difficult to manage consequence of HZ. Early recognition and treatment of $\mathrm{HZ}$ can reduce acute symptoms and may also reduce PHN. Having a sound understanding of the prevention, diagnosis and treatment of $\mathrm{HZ}$ is important in reducing the impact of the burden of disease for patients and their families. Immunization creates the opportunity to shift disease management from treatment to prevention. Currently, a vaccine for Herpes Zoster is approved for use in Canada for the prevention of herpes zoster(shingles) in individuals 50 years of age or older. This educational session focuses on Herpes Zoster burden of disease, clinical manifestations, as well as the appropriate use of the $\mathrm{HZ}$ vaccine.

\section{KEYNOTE SPEAKERS - 9:15 AM}

\section{1}

Chair: Christine T Chambers

Dalhousie University \& IWK Health Centre, Halifax, Nova Scotia

\section{A}

THIS WON'T HURT A BIT: REFLECTIONS ON A "PAINFUL" CAREER

\section{CANADIAN PAIN SOCIETY DISTINGUISHED CAREER AWARD}

RECIPIENT

Joel Katz

Department of Psychology, York University, Department of Anesthesia and Pain Management, Toronto General Hospital and University of Toronto, Toronto, Ontario

The prospect of undergoing major surgery can be daunting. Among the concerns about postoperative pain are how intense it will be, whether it will be managed effectively, and how long it will last. This presentation will provide an overview of chronic post-surgical pain (CPSP), including some of the more intractable conditions such as post-amputation phantom limb pain, post-thoracotomy pain; basic epidemiological data; known risk and protective factors; and a look to the future. Throughout the presentation Dr. Katz will focus on research he and his colleagues and students have conducted over the past 30 years.

Learning Objectives:

After attending the session participants will be able to:

1. Specify the incidence/prevalence of CPSP in several high risk surgical populations;

2. Distinguish between causal and correlated risk factors;

3. List the known risk and protective factors for the development of chronic post-surgical pain.

\section{B}

PERSISTENT CARDIAC PAIN: A BURGEONING SCIENCE REQUIRING A NEW APPROACH CANADIAN PAIN SOCIETY EARLY CAREER AWARD RECIPIENT Michael McGillion

University of Toronto, Lawrence S Bloomberg Faculty of Nursing, Toronto, Ontario

Dr McGillion's research focuses on improving access to appropriate healthcare for people suffering from persistent forms of cardiac pain including refractory angina and non-ischemic chest pain following successful percutaneous coronary interventions. Specifically, his research targets clinical decision support and self-management interventions as well as innovative approaches to cardiac pain education and knowledge dissemination.

Learning Objectives:

1. To understand the underlying theory and process for development and evaluation of a decision support tool for the treatment of refractory angina.

2. To understand the process for development and evaluation of a multi-media, web-based resource centre for large-scale dissemination of persistent cardiac pain-related knowledge.

3. To understand the pedagogic design and evaluation of a complex simulation intervention to improve assessment and management of cardiac pain.

\section{SESSION $301-11: 15$ AM}

\section{2}

PEER-TO-PEER SUPPORT FOR PEOPLE WITH CHRONIC PAIN: THE NEED, THE DELIVERY MODELS, AND THE NEXT STEPS

Chair: James L Henry

Speakers: Glen Hutzul; Paula Forgeron; James L Henry;

Sara Ahola Kohut

WORKSHOP OBJECTIVE:

The objectives of this symposium are to provide an update on current research on peer-to-peer support for people who live with chronic pain and to capture perspectives on peer-to-peer support initiatives, exploring the need, the methods of delivery and recommendations for future initiatives and research.

Learning Objectives:

1. From this symposium those attending should understand the theories of social support and the importance of peer support programs for people living with chronic pain

2. From this symposium those attending should understand the current state of research on the effectiveness of peer support programs for youth and adults with chronic pain and lessons learned

3. From this symposium those attending should understand the perspectives from consumers living with chronic pain

\section{A}

\section{STUMBLING BLOCKS ON THE ROAD TO WELLNESS FOR CHRONIC PAIN PATIENTS}

\section{Glen Hutzul}

ACTION PNP Committee

From both firsthand patient perspective and in his role facilitating a chronic pain support group, Glen will discuss how peer to peer support aids in helping chronic pain patients overcome common stumbling blocks on the road to wellness. These stumbling blocks include isolation, depression, poverty and hopelessness. This presentation from the patient's perspective 
includes scenes from a documentary where two patients with severe chronic pain highlight the psychosocial issues they faced.

\section{B \\ LIVING WITH DIFFERENCE: CHALLENGES TO FRIENDSHIPS FOR ADOLESCENTS WITH PAIN \\ Paula Forgeron}

University of Ottawa, Ottawa, Ontario

For most people social support is secured within their own naturally occurring social network, which can range from family and friends to church groups and co-workers. During adolescents friendships become a major source of social support and increase in importance. Establishing and maintaining close friendships is a hallmark of adolescence and these friendships are developmental critical as they provide a milieu to develop and test beliefs and values that contribute to self-identity. Chronic pain can interrupt the establishment and maintenance of friendships beyond the interference with school attendance and activities. Negative interactions with friends can lead to decreased social engagement. Findings from several studies will be presented and include the friendship experiences of adolescent's with chronic pain, ranges in adolescents desire to befriend another adolescent with chronic pain, and differences between adolescents with chronic pain and healthy controls in their interpretation of social situations. The goal of this presentation is to examine potential factors that facilitate and challenge adolescents with chronic pain to secure their social support needs from their close friends and highlight potential strategies to help these adolescents establish and maintain these critical friendships.

\section{C}

TOWARD HABERMAS' THEORY OF COMMUNICATIVE ACTION: LESSONS FROM A CHRONIC PAIN SUPPORT GROUP

\section{James L Henry}

\section{McMaster University, Hamilton, Ontario}

Research provides knowledge of the mechanisms, complexities, incidence and prevalence of chronic pain, and the personal, social and economic costs. Yet important questions remain: is this knowledge being applied successfully to those who live with disabling pain; are we using all effective means to apply this knowledge? This presentation is an extraction of experiences by a systems neurophysiologist who has also made personal observations of a qualitative nature in running a community-based Chronic Pain Support Group for the past eight years. Experiences teach in a direction imperceptible to a basic scientist. Group participants interact well within the group but a gap exists with 'outsiders', whether family, friend, pharmacist, physician, nurse, police officer, insurer, etc. Further, the pain experience is different, whether constant and predictable or episodic and unpredictable, but all chronic pain tends to be conveniently packaged as a unity, for study and understanding. Among peers personal differences are accepted; successes, failures and conventional or nonconventional methods of dealing with chronic pain are expressed, shared and critiqued. Friendships are established. Participants return monthly to share feelings, to network socially, to express themselves. Experience has taught that a community-based chronic pain support group is an untapped resource of tacit as well as embedded knowledge, constituting social capital that remains to be brought into the mainstream of pain research and management. Though in a different context, Habermas (1984) has suggested a concept of communicative action where members of a society reach common understanding by reasoned argument, consensus and cooperation rather than a strategic action strictly in pursuit of particular or unique goals. This concept should be applied here. It is crucial to include this social capital within the breadth of pain research and management, to apply, refine, measure and evaluate this knowledge as an effective means to improve the lives of individuals with chronic pain.

\section{D}

\section{VIRTUAL PEER-TO-PEER SUPPORT FOR YOUTH WITH} CHRONIC PAIN

\section{Sara Ahola Kohut}

\section{Hospital for Sick Children, Toronto, Ontario}

Chronic pain in adolescents is a common problem. However, many youth with chronic pain face numerous barriers (e.g., no services available in many geographic areas, long waitlists, and limited availability of trained professionals in non-urban centers) to accessing services and peer support. Today, youth frequently use the Internet in order to communicate with peers, whether to strengthen existing relationships or to develop new ones. Internet-based interventions can therefore provide an innovative approach to improve the accessibility of treatment programs for youth with chronic illnesses. In this session I will discuss the development and initial evaluation of a virtual peer-to-peer support intervention for youth with chronic pain. First, in order to highlight the essential components successful interventions, a systematic review of peer-to-peer supports for youth with chronic health conditions will be discussed. Next, the structure of a manualized peer-to-peer support program for youth with chronic pain will be described. This peer-to-peer intervention will connect trained young adults living with chronic pain to adolescents struggling to manage their chronic pain. Participants will connect once a week for the 12-week study period using virtual mediums (Skype). This peerto-peer intervention will provide social support, encouragement, and education for effective chronic pain self-management strategies. Outcome measures include physical symptoms, emotional symptoms, coping efficacy, self-efficacy, health related quality of life, social support, and selfmanagement. Lastly, preliminary results of pilot testing will be discussed. The proposed research aims to improve the access and acceptability of self-management and social support treatments of adolescents living with chronic pain.

SESSION $302-11: 15$ AM

\section{3}

\section{PARENTS AND FAMILIES AS ACTIVE PARTICIPANTS IN PAIN MANAGEMENT ACROSS THE AGE SPAN}

Chair: Line Caes

Speakers: Line Caes; Marsha Campbell-Yeo; Michael Sullivan WORKSHOP OBJECTIVE:

To identify challenges and potential solutions to involve parents and families as effective, active participants in pain management across the age span.

Learning Objectives:

1. Review the evidence regarding the efficacy of parent and family involvement in neonatal, pediatric and adult population.

2. Identify parents and families at risk and challenges of optimal involvement, which may vary across the age span.

3. Discuss the feasibility of implementation and potential solutions.

\section{A}

OPTIMAL INVOLVEMENT OF PARENTS IN PEDIATRIC PAIN MANAGEMENT: THE ROLE OF CATASTROPHIC THINKING Line Caes

Centre for Pediatric Pain Research, IWK Health Centre, Halifax, Nova Scotia

To date, a lot of progress has been made to reduce child pain and fear during painful medical procedures. Accumulating evidence indicates that the way parents respond to child pain is critically important to how children attempt to cope with pain. Although parental reactions are not always adaptive, little is known about which parents need assistance to cope effectively with child pain. Additionally, evidence about the beneficial effects of parental attendance on child's anxiety is mixed. While some studies report reduced child anxiety when parents are present, other studies found heightened levels of anxiety or no significant impact of parental presence. It is likely that the optimal involvement of parents in pediatric pain management may depend upon parental characteristics. The current presentation will focus on the role of parental catastrophic thoughts about child pain, thereby discussing results of studies in healthy schoolchildren and children with leukemia undergoing a lumbar puncture. Our findings suggest that interventions to enhance optimal involvement of parents might be especially advised for parents who endorse higher levels of catastrophic thoughts about child pain. Specifically, although high catastrophizers show an increasing desire to be present, their heightened tendency to engage in protective behavior may negatively impact the child's pain experience. However, little is known on how to assist high catastrophizing 
parents to effectively cope with their child's pain. Several potential solutions to enhance the effectiveness of high catastrophizing parents' involvement in pediatric pain management (e.g., targeting parental catastrophic thoughts; providing effective emotion regulation strategies) will be discussed.

\section{B}

\section{MATERNAL AND FAMILY DRIVEN ANALGESIA IN THE NICU} - EVIDENCE, CHALLENGES AND IMPLEMENTATION

Marsha Campbell-Yeo

Dalhousie University School of Nursing and IWK Health Centre, Halifax, Nova Scotia

All infants experience pain. However, those delivered preterm or critically ill are particularly vulnerable as they undergo numerous repeated painful procedures as part of their medical care in the NICU. This exposure is associated with both short and long-term sequelae affecting sensation, perception and neurocognition and the use of pain relieving strategies such as sweet taste and non-nutritive sucking are commonly used. However, an evolving paradigm shift embracing a family-centered care philosophy has precipitated a rapidly growing interest in using mothers and families as agents of comfort in the NICU setting. This has been based on several premises: the loss of comfort-providing role of parents in critical care settings; the positive effects of maternal contact on various parameters of neonatal stability and state regulation; and, consistent evidence demonstrating the efficacy of maternal driven pain relieving strategies, specifically breastfeeding and maternal skin-to-skin contact (SSC) on the reduction in behavioral pain response during minor painful procedures. We will provide an overview of the current evidence for breastfeeding and also results from our recent Cochrane systematic review regarding maternal SSC as a pain relieving intervention including a summary of the effectiveness of non-maternal surrogates. Interestingly, despite consistent positive results, these maternal driven interventions are significantly under utilized. Little is known about this lack of involvement; although, preliminary findings indicate that the perceptions of care providers and parents regarding family participation are incongruent. Contextual issues related to feasibility, identified challenges and barriers, and clinical uptake strategies will be discussed.

\section{C}

\section{DYADIC PROCESSES IN CHRONIC PAIN}

\section{Michael Sullivan}

Departments of Psychology, Medicine and Neurology, McGill

University, Montreal, Quebec

Chronic pain brings challenges not only to the individual with pain, but to the spouse as well. Numerous investigations have documented the physical and emotional toll that chronic pain can have on spouses. Increased recognition of the impact of chronic pain on dyadic relations prompted research examining the effectiveness of spousal involvement in the treatment of individuals with chronic pain. The results of several clinical trials suggest that the involvement of spouse in pain management interventions can yield positive benefits for the individual with chronic pain as well as the spouse. Clinical accounts however indicate that spousal involvement in pain management interventions does not always yield benefit. Factors such as the quality of the marital relationship, spousal levels of catastrophizing and depression play a role in determining whether spousal involvement will foster or hinder a client's progress in a pain management intervention. This presentation will review recent literature on dyadic processes in adjustment to chronic pain as well research findings documenting the effectiveness of spousal involvement in chronic pain. In addition, case studies will be presented to highlight potential challenges and drawbacks to involving spouses in pain management interventions.

\section{SESSION $303-11: 15$ AM}

\section{4}

\section{CRITICAL PERSPECTIVES OF PSYCHOSOCIAL CONCEPTS IN PAIN}

Chair: Thomas Hadjistavropoulos

Speakers: Kenneth M Prkachin; Thomas Hadjistavropoulos;

Jennifer Stinson

WORKSHOP OBJECTIVE:

With a focus on both adults and children, the purpose of this symposium will be to shine a critical light on psychosocial concepts that influence clinical practice, with a view to identifying their areas of strength, weakness and need for further investigation.

Learning Objectives:

1. To familiarize participants with biopsychosocial formulation of the pain experience (e.g., the fear avoidance model of pain).

2. To familiarize participants with limitations of the empirical base of specific psychosocial formulations.

3. To highlight frequent misapplications of psychosocial constructs in clinical practice.

\section{A}

USE AND MISUSE OF THE CONCEPT OF PAIN BEHAVIOUR Kenneth M Prkachin

Department of Psychology, University of Northern British

Columbia, Prince George, British Columbia

The notion of a "biopsychosocial" model has by now received widespread acceptance among pain clinicians and researchers. Inherent to the biopsychosocial approach is the idea that behaviours linked to pain (pain behaviours) allow us to understand the nexus of converging biological, psychological and social forces. This idea hides conflicting interpretations of the meanings of pain behaviours and implies a greater degree of understanding than can be justified. In particular, clinical interpretations of the meaning of pain behaviours can be widely divergent, with potentially unfortunate consequences for those subject to such interpretations. This presentation will address potential pitfalls in the clinical interpretation of pain behaviours. Examples of clinical interpretations of pain behaviour from the literature and from case histories will be discussed from the perspective of the assumptions about the meaning of pain behaviours that they convey. Comparison will be made with current scientific evidence, where it exists, to evaluate whether the derived interpretations are warranted. Areas where research is needed will also be identified. The discussion will support the conclusion that there is a substantial disconnect between what can be asserted scientifically and what is asserted clinically about the nature of pain behaviours.

\section{B}

LIMITATIONS IN THE EMPIRICAL SUPPORT FOR THE FEAR AVOIDANCE MODEL OF PAIN

Thomas Hadjistavropoulos

Department of Psychology and Centre on Aging and Health. University of Regina, Regina, Saskatchewan

The fear avoidance model of pain has been highly influential in directing rehabilitation and research. It postulates that when activity becomes associated with pain, some individuals experience heightened anxiety and fear which lead to excessive avoidance. In turn, such avoidance leads to deconditioning and negatively impacts rehabilitation. The role of cognition (e.g., catastrophic thinking about pain) is also recognized in the model. Despite its popularity and significant research base, however, aspects of the model are lacking adequate empirical support. For example, longitudinal research has yet to confirm convincingly that excessive avoidance results in poorer rehabilitation outcomes. Moreover, after adjusting for injury severity, longitudinal research has yet to confirm that fear of pain is an independent risk factor for disability. Strengths and rarely discussed limitations of the model will be outlined along with a discussion of clinical implications and important avenues for future research. 
24C

\section{APPLICATIONS AND MISAPPLICATIONS OF THE PEDIATRIC FEAR AVOIDANCE MODEL OF PAIN \\ Jennifer Stinson}

Hospital for Sick Children and Lawrence S Bloomberg Faculty of Nursing and Department of Pediatrics, University of Toronto, Toronto, Ontario

Problems inherent in downward extension of adult models of pain and associated findings to the unique context of chronic pain in children will be outlined. The Pediatric Fear-Avoidance Model of Chronic Pain (Asmundson et al., in press) will be presented outlining the child factors and the role of parents, specifically the reciprocal relationships between parent psychological and behavioural factors and child psychological and behavioural factors. A case study will be used to highlight aspects of the model in terms of its applicability to guide assessment and treatment. The model's research base, limitations and future directions for research in pediatric chronic pain will discussed.

\section{REFERENCES:}

1. Asmundson, G. J. G., Noel, M., Petter, M., \& Parkerson, H. (In press). Pediatric fear-avoidance model of pain: Foundation, application, and future directions. Pain Research and Management

\section{SESSION $304-2: 15$ PM}

\section{5}

\section{THE IMPACT OF PERCEIVED INJUSTICE AND ACCEPTANCE} ON CHRONIC PAIN OUTCOMES: EVIDENCE, PROCESSES,

\section{AND TREATMENT}

\section{Chair: Anita Unruh}

Professor and Associate Dean (Research \& Academic), Faculty of Health Professions, Dalhousie University, Halifax, Nova Scotia Speakers: Michael JL Sullivan; Whitney Scott; Charles Nelson WORKSHOP OBJECTIVE:

This symposium will bring together data examining the impact of two psychosocial constructs, perceived injustice and acceptance, on pain outcomes. The first speaker will examine the applicability of the construct of perceived injustice to the chronic pain context. This presentation will also review published research that indicates perceived injustice is a risk factor for adverse pain-related outcomes. The second speaker will explain the application of a conceptual model based on principles of Acceptance and Commitment Therapy (ACT) to understand the association between perceived injustice and problematic pain outcomes. Data examining the role of acceptance as a potential mediating process between perceived injustice and adverse pain outcomes will be presented. The third speaker will describe the utility of ACT for treating complex chronic pain in a sample of Canadian military personnel and veterans with posttraumatic stress disorder (PTSD). This presentation will explain how ACT was successfully adapted for group psychotherapy, and will provide pre- and post-treatment outcomes relating to pain intensity, anxiety, catastrophizing, and acceptance.

Learning Objectives:

1. Attendees will be informed about research demonstrating the adverse health and mental health outcomes associated with perceived injustice. Attendees will gain an understanding of the clinical assessment of perceived injustice and potential interventions strategies targeting injustice among patients with chronic pain.

2. Attendees will gain an understanding of the applicability of an ACT-based conceptual model to explain the impact of perceived injustice in chronic pain. Attendees will be familiarized with emerging research examining the relationship between perceived injustice and acceptance of chronic pain.

3. Attendees will be able to describe the components of a successful group therapy program using ACT to treat complex chronic pain. Additionally, attendees will improve their understanding of the relationship between pain and PTSD and how ACT can be used to assist in the reduction of pain and PTSD symptoms.

\section{A}

\section{THE IMPACT OF PERCEIVED INJUSTICE ON CHRONIC PAIN OUTCOMES \\ Michael JL Sullivan \\ McGill University, Montreal, Quebec}

This presentation will examine the experience of perceived injustice in chronic pain. The measurement of pain-related injustice perceptions will be discussed. Results from questionnaire studies will be reviewed to describe the negative impact of perceived injustice on pain-related physical and psychological functioning in patients with persistent pain. Recent results from experimental studies will also be discussed to highlight potential mechanisms underlying the relationship between perceived injustice and pain outcomes. Potential processes mediating the relationship between perceived injustice and pain outcomes, including blame attributions, rumination, retribution motives and anger will be examined. Implications for intervention will be discussed.

\section{B}

\section{THE APPLICATION OF A CONCEPTUAL MODEL OF PSYCHOLOGICAL FLEXIBILITY TO UNDERSTAND PERCEIVED INJUSTICE IN CHRONIC PAIN}

\section{Whitney Scott}

McGill University, Montreal, Quebec

This presentation will put forward a conceptual model of perceived injustice in chronic pain based on principles of Acceptance and Commitment Therapy (ACT). Processes of psychological (in)flexibility will be proposed as factors that may mediate the relationship between perceived injustice and pain outcomes. Based on a psychological flexibility model, proposed mediating factors will include cognitive fusion, non-acceptance, disconnect from the present, non-values based action and lack of committed action. Prospective questionnaire data will be presented to examine whether processes of psychological inflexibility mediate between perceived injustice and pain outcomes. This conceptual model and preliminary data will be discussed in terms of implications for intervention.

\section{C}

\section{A PRELIMINARY STUDY OF THE EFFECTIVENESS OF GROUP ACCEPTANCE AND COMMITMENT THERAPY FOR COMPLEX CHRONIC PAIN AND POSTTRAUMATIC STRESS DISORDER}

\section{Charles Nelson}

University of Western Ontario, London, Ontario

Acceptance and commitment therapy (ACT) aims to help patients with chronic pain accept painful sensations as a part of daily life and to maintain as active and "normal" a life as possible without restructuring daily activities to accommodate pain. The objectives of this presentation are: 1) to describe how ACT was adapted as a group therapy modality for a cohort of treatment-seeking Canadian Forces members and veterans with posttraumatic stress disorder (PTSD); and 2) to provide pre- and post-treatment outcomes relating to pain intensity, anxiety, catastrophizing, and acceptance. Clients of the Parkwood Hospital Operational Stress Injury clinic with a diagnosis of PTSD and chronic pain were invited to join an ACT group for chronic pain. Participants completed a number of pain-related self-report questionnaires at baseline and immediately post-treatment. Participants reported greater pain acceptance and lower pain intensity, catastrophizing, and anxiety post-treatment. The results of this preliminary study suggest that: 1) ACT can successfully be adapted to a group format; and 2) ACT appears to be a beneficial treatment modality for individuals with PTSD and comorbid complex chronic pain. 


\section{6}

\section{SPINAL FACET JOINT PAIN: HUMAN AND ANIMAL STUDIES OF BASIC MECHANISMS}

\section{Chair: Howard Vernon}

Speakers: Howard Vernon; James Henry; Beth Winkelstein

\section{WORKSHOP OBJECTIVE:}

The aim of this Symposium is to provide an update on the status of facet joint pain in the context of low back and neck pain in society. We propose to review clinical evidence and novel animal models investigating the lumbar and cervical facet joints with respect to pain and sensory-motor mechanisms.

Learning Objectives:

1. Neuroanatomical details related to the facet joints which help explain the unique features of deep spinal pain: pain referral patterns, poor localization of pain and which contribute to the well-known chronicity and recurrence of low back pain.

2. Different methodologies for exploring facet joint pain in rat models.

3. Potential mechanisms of pain generation from the lumbar and cervical facet joints, resulting from different clinically-relevant aetiologies including osteoarthritis and trauma. Future applications of these studies will be discussed towards translational benefits.

\section{A \\ MECHANISMS OF FACET-BASED PAIN \\ Howard Vernon}

Canadian Memorial Chiropractic College, Toronto, Ontario

BACKGROUND: The mechanisms subserving deep spinal (facet/deep muscles) pain have not been studied as well as those related to the skin and to deep pain in peripheral limb structures. The clinical phenomenology of deep spinal pain presents unique features which call for investigations which can explain these at a mechanistic level.

METHODS: Targeted searches of the literature were conducted and the relevant materials reviewed for applicability to the thesis that deep spinal pain is distinctive from deep pain in the peripheral limb structures.

RESULTS: Since the 1980's the innervation characteristics of the spinal facet joints, intervertebral discs and deep muscles have been explored. Afferent connections subserving pain have been identified in a distinctive somatotopic organization within the spinal cord whereby afferents from deep spinal tissues terminate primarily in the lateral dorsal horn while those from deep peripheral tissues terminate primarily in the medial dorsal horn. Mechanisms underlying the clinical phenomena of referred pain from the spine, poor localization of spinal pain, and chronicity of spine pain have emerged from the literature and are reviewed here, especially emphasizing the somatotopic organization and hyperconvergence of dorsal horn "low back (spinal) neurons". Taken together, these findings provide preliminary support for the hypothesis that deep spine pain is different from deep pain arising from peripheral limb structures.

\section{B}

\section{A NOVEL RAT MODEL OF LUMBAR FACET INJURY James Henry} McMaster University, Hamilton, Ontario

STUDY DESIGN: Controlled animal experiment.

OBJECTIVE: To develop a novel, persisting model of lumbar facet joint pain and to determine histological, behavioral and biochemical changes.

METHODS: Adult male Sprague Dawley rats were anaesthetized and the L5/L6 facet joint was exposed unilaterally. In model animals, the joint was compressed to $\sim 1 \mathrm{~mm}$ with modified clamps applied for three minutes; sham-operated animals were also used. Following surgery, animals were tested 7, 14, 21 and 28 days later for nociceptive scores using von Frey filaments applied to the hind paws and deep tissue sensitivity using a blunt algometer applied to lumbar paravertebral tissues. Different groups were sacrificed at these same times for histological and biochemical analysis of tissues.

RESULTS: Histological sections revealed site-specific loss of cartilage in model animals without any clear signs of change in bony structures. For von Frey testing, post-model reductions were obtained for the ipsi- and contralateral paws which lasted to 28 days. For pressure algometry, postmodel reductions of vocalization thresholds were obtained at all three sites up to 28 days, with the $\mathrm{L} 2$ site showing the greatest reduction. Biochemical analyses revealed increases in pro-inflammatory cytokines, especially TNF- $\alpha$ and IL- $1 \beta$.

CONCLUSIONS: These data suggest that unilateral compression of a facet joint induces a novel model of local loss of cartilage accompanied by increased sensitivity to regional mechanical stimuli and by increases in inflammatory mediators. This new model may prove to be useful for studies on mechanisms and treatment of lumbar facet joint pain and osteoarthritis.

\section{C}

\section{CERVICAL FACET JOINT INJURY: REVIEW OF ANIMAL} STUDIES

Beth Winkelstein

University of Pennsylvania, Philadelphia, Pennsylvania

STUDY DESIGN: Controlled animal experiment.

OBJECTIVE: To utilize an in vivo model of mechanical injury to the cervical facet joint to investigate mechanisms of persistent pain onset and maintenance and to identify potential effective therapeutics for facet joint pain.

METHODS, RESULTS \& DISCUSSION: The lecture will provide an overview of biomechanical, physiologic, and species-related considerations for modeling painful facet joint injury in vivo. Relevant human and animal anatomy will be reviewed and discussed in the context of animal models. A review of the current schema of how pain develops from a facet joint lesion, whether from trauma or degeneration, will be reviewed considering clinical data and basic science research. In particular, the joint cartilage and enclosing capsular ligament will be the focus since they both have key roles in the function of the normal, injured and pathologic joint. Basic facet joint biomechanics will also be presented in order to provide appropriate context for its role in injury and pain. Specific neuroimmune regulation in the spinal cord will be presented in the context of facet joint pain. This presentation will emphasize the appropriate background for understanding the facet joint's involvement in normal and pathological function of the spine and will provide a working framework for understanding how this joint contributes to pain. Complementary research approaches spanning cadaveric biomechanical studies and our in vivo pain model will be presented to provide a comprehensive presentation of basic and applied research findings related to facet-mediated pain, including potential therapeutic approaches.

\section{SESSION $306-2: 15$ PM}

\section{7}

\section{PREVENTION OF CHRONIC POST-SURGICAL PAIN: FROM BENCH TO BEDSIDE}

Chair: Joel Katz

Speakers: Simon Beggs; Joel Katz; Hance Clarke WORKSHOP OBJECTIVE:

Chronic post-surgical pain (CPSP) is now recognized as an adverse outcome of surgery. The occurrence of CPSP varies according to the surgical procedure; surgeries that result in damage to peripheral nerves - whether intention or accidental - are typically associated with a higher incidence and intensity of CPSP suggesting that the problem is mainly neuropathic in nature. Nevertheless, other factors are clearly involved since not all patients with nerve damage develop CPSP and not all CPSPs involve nerve damage. Moreover, we are a long way from being able to predict who will develop CPSP and who will recover uneventfully. The overall aims of this workshop are (1) to review the neurobiological mechanisms underlying chronic neuropathic pain with an emphasis on novel neuron-glial interactions that contribute to physiological and pathological processes in the central nervous system and ultimately to pain; (2) to present what is known about the risk and protective factors that predict the transition of acute pain to CPSP; and (3) to provide evidence for and against the possibility that administration of various analgesic agents during the perioperative period will reduce the incidence or intensity of CPSP. 
Learning Objectives:

1. To understand the neurobiological mechanisms underlying chronic neuropathic pain.

2. To consider the risk and protective factors associated with CPSP

3. To identify preventive approaches to the management of perioperative pain that might lessen the risk of developing CPSP

\section{A}

THE PATHOBIOLOGY OF CHRONIC NEUROPATHIC PAIN: IMPLICATIONS FOR PREVENTION

Simon Beggs

Programmes in Brain and Behaviour, Hospital for Sick Children and Faculty of Dentistry, University of Toronto, Toronto, Ontario

Considerable evidence now shows that chronic pain is not simply persistent acute pain. Rather, the underlying neurobiological mechanisms of the two are fundamentally different: acute pain is a physiological function of the normal nervous system, whereas chronic pain, in particular chronic neuropathic pain, is a consequence of pathological alterations to both peripheral and central nervous systems. Within the spinal cord nociceptive network a change in the balance of excitatory and inhibitory influences underlies the emergence and persistence of the cardinal symptoms of neuropathic pain. While the transmission of nociceptive information from the periphery to the brain is carried by neurons, neuron-glial interactions are increasingly recognized as being key for physiological and pathological processes in the central nervous system. Microglia in the spinal dorsal horn respond to injury to peripheral nerves by adopting a specific response state characterized by upregulation of the purinergic receptor P2X4. In this $\mathrm{P} 2 \mathrm{X} 4 \mathrm{R}+$ state microglia release brain derived neurotrophic factor (BDNF) which disinhibits neurons in the spinal nociceptive processing network. The transformation in processing caused by signalling of P2X4R + microglia to nociceptive transmission neurons may account for the main symptoms of neuropathic pain in humans. The identification of interactions between neuronal and glial populations in the CNS reveals potential new therapeutic targets to specifically block the central cellular and molecular mechanisms that mediate the transition from acute to chronic pain.

\section{B}

\section{RISK AND PROTECTIVE FACTORS ASSOCIATED WITH} CHRONIC POST-SURGICAL PAIN

\section{Joel Katz}

Department of Psychology, York University, Department of Anesthesia and Pain Management, Toronto General Hospital and University of Toronto, Toronto, Ontario

Chronic postsurgical pain (CPSP) develops in an alarming proportion of patients. Dr. Katz will review the epidemiology of chronic postsurgical pain. Biopsychosocial risk factors that predict the transition of acute pain to CPSP will then be presented, highlighting surgical, psychosocial, socioenvironmental, and patient-related factors that appear to confer a greater risk of developing chronic postsurgical pain. The rationale for a preventive multimodal analgesic approach to surgery will be presented emphasizing important methodological issues in the design of clinical studies. The distinction between causal and non-causal risk factors is discussed with specific reference to the development of CPSP and its prevention. Although we are beginning to understand the factors that facilitate and protect against the development of CPSP, the transition of acute postoperative pain to CPSP remains a complex and poorly understood developmental process involving biological, psychological and social-environmental factors.

\section{C}

THE PREVENTION OF CHRONIC POSTSURGICAL PAIN: RECENT FINDINGS

\section{Hance Clarke}

Department of Anesthesia and Pain Management, Toronto General Hospital and University of Toronto, Toronto, Ontario

The development of chronic postsurgical pain (CPSP) is an unfortunate consequence of surgery that adversely impacts the patient's quality of life.

Efforts to prevent the establishment of CPSP include perioperative administration of a variety of pharmacological agents. The most consistent patient factor that contributes to the development of CPSP is the presence and/or intensity of preoperative and postoperative pain. Because moderate to severe postoperative pain is a frequent occurrence after surgery, novel pharmacological agents such as the alpha-2-delta-ligands, gabapentin and pregabalin, in addition to traditional analgesic agents are administered with the aim of providing superior pain relief at rest and with movement; reducing opioid consumption and reducing analgesic-related adverse effects. If pharmacological agents, such as gabapentin and pregabalin, can prevent the establishment of surgery-induced central sensitization and can decrease postoperative pain, then these drugs, given during the perioperative period, may also play a role in preventing the transition of acute pain to chronic pain. Dr. Clarke will review recent clinical evidence concerning the alpha-2-delta-ligands and their effects on preoperative anxiety, postoperative pain and the prevention of CPSP. 
P1

\section{THE PROGNOSTIC VALUE OF PERCEIVED INJUSTICE AND DEPRESSION FOR LONG TERM PAIN AND DISABILITY \\ Hayley Ali $^{1,2}$; Whitney Scott ${ }^{2}$; Michael JL Sullivan ${ }^{2}$ \\ ${ }^{1}$ Cardiff University, Cardiff, Wales; ${ }^{2}$ McGill University, Montreal, Quebec}

AIM: Previous research has highlighted the adverse affects of perceived injustice and depression on recovery trajectories following injury. Little however is known about the distribution of perceived injustice and depression scores in individuals with musculoskeletal conditions, or the independent and combined adverse influences of these variables on recovery outcomes.

This study examined the independent and combined adverse effects of perceived injustice and depression on recovery outcomes (12 month follow up pain severity and work status) following musculoskeletal injury.

METHODS: The sample consisted of 169 (106 women, 63 men) who had sustained soft tissue injuries to the lower back in an occupational incident. Scores on measures of perceived injustice and depression were dichotomized in relation to clinical threshold cut scores

RESULTS: Fifty five percent of the sample scored below clinical threshold on both perceived injustice and depression. Twenty percent of the sample scored high on perceived injustice; of these, the majority (77\%) also scored above clinical threshold on the measure of depression. Compared to individuals with scores below clinical threshold, participants with high perceived injustice scores had an increased probability of long-term work-disability, and an increased probability of developing chronic pain. The presence of depression had a cumulative effect on the chronicity of pain and work-disability.

CONCLUSIONS: Individuals with high perceived injustice scores are also likely to score above clinical threshold on measures of depression. High scores on both perceived injustice and depression appear to have a particularly strong negative impact on recovery outcomes following back injury. The findings argue for the inclusion of measures of depression in the routine screening of individuals with back injuries, and the incorporation of treatments targeting perceived injustice and depression in primary care services.

\section{P2}

\section{JUST WORLD BELIEFS MODERATE THE RELATION BETWEEN PAIN-RELATED INJUSTICE PERCEPTIONS AND DEPRESSIVE SYMPTOMS IN INDIVIDUALS WITH CHRONIC PAIN}

Elena Bernier ${ }^{1}$; Whitney Scott ${ }^{1}$; Zina Trost $^{2}$ Michael JL Sullivan ${ }^{1}$ ${ }^{1}$ McGill University, Montreal, Quebec; ${ }^{2}$ University of North Texas, Denton, Texas, USA

AIM: Emerging research suggests that pain-related perceptions of injustice adversely impact mental health outcomes associated with chronic pain. It has been suggested that the adverse impact of perceived injustice might be buffered by the degree to which individuals believe in a just world (JWB). To date, research has not examined the combined impact of pain-related injustice perceptions and JWB on mental health outcomes. The purpose of this study was to examine whether pain-related perceptions of injustice and JWB interact to predict depressive symptoms.

METHODS: One hundred and sixty-nine individuals with chronic musculoskeletal pain participated in this study. Participants completed selfreport measures of perceived injustice, personal and general just world beliefs, and depressive symptoms. Based on median splits, participants were identified as being high or low on the measures of perceived injustice and just world beliefs. The following groups were created: high injustice, high JWB; high injustice, low JWB; low injustice, high JWB; and, low injustice, low JWB.

RESULTS: Results indicated that individuals with high perceived injustice and high personal JWB had significantly lower depressive symptoms than individuals with high perceived injustice and low personal JWB, $t(167)=-2.75, p<0.01$. Although marginally significant $(\mathrm{p}=0.06)$, a similar trend was seen for general beliefs in a just world.

CONCLUSIONS: These results suggest that the ability to maintain a belief in a just world may protect against adverse mental health outcomes associated with perceiving pain-related injustice. Implications for the conceptual understanding of perceived injustice in chronic pain will be discussed.

\section{P3}

A QUALITATIVE EXPLORATION OF SPIRITUALITY/RELIGION AND COPING WITH CHRONIC PAIN: FOUR ILLUSTRATED JOURNEYS

Kathryn A Birnie ${ }^{1}$; Anita Unruh ${ }^{2}$; Mary Lynch ${ }^{3}$

${ }^{1}$ Department of Psychology; ${ }^{2}$ School of Health and Human Performance; ${ }^{3}$ Departments of Psychiatry, Anesthesia, and Pharmacology, Dalhousie University, Halifax, Nova Scotia

AIM: Over $80 \%$ of Canadians identify as religious. However, spirituality/ religion remains an understudied area in chronic pain despite evidence demonstrating its critical influence on psychological and physical wellbeing. The aim of this research was to explore the role of spirituality/religion for individuals coping and managing with chronic pain.

METHODS: Four women with chronic pain participated in two individual interviews. These participants were selected from a larger group to represent differing emphases of spirituality/religion in a person's life prior to the onset of chronic pain. Participants described themselves as spiritual (with no religious affiliation) or Catholic, and two were Sisters of Charity (Catholic nuns). They were 42 to 86 years of age. Time living with chronic pain ranged from 8 to 30 years and was due to arthritis, fibromyalgia, accident/injury, and disease/surgery. Interviews were transcribed verbatim and analyzed using an interpretive phenomenological approach.

RESULTS: Interviews suggested six themes: pain as part of life, spirituality/religion as integral to coping well, reclaiming self through acceptance and acknowledgement of pain, staying present focused, developing a clearer perspective, and maintaining an active role in managing pain. Although common themes were identified, the meaning of chronic pain in each life was framed by how integrated spirituality/religion had been to their sense of self prior to chronic pain.

CONCLUSIONS: This study illustrates how spirituality/religion offers a particular adaptive role for individuals with chronic pain who identify such worldviews. These findings extend current research by highlighting the influence of previously held spiritual/religious frameworks within which individual's understand their experience of chronic pain.

\section{P3A}

INFLUENCE OF WATER TEMPERATURE AND APPARATUS ON PAIN-RELATED OUTCOMES FOR CHILDREN COMPLETING THE COLD PRESSOR TASK

Kathryn A Birnie $^{1,2}$; Jennifer A Parker ${ }^{2}$; Christine T Chambers ${ }^{2,3}$ ${ }^{1}$ Department of Psychology, Dalhousie University; ${ }^{2}$ Centre for Pediatric Pain Research, IWK Health Centre; ${ }^{3}$ Departments of Psychology and Pediatrics, Dalhousie University, Halifax, Nova Scotia

AIM: The cold pressor task (CPT) is a commonly used experimental pain task with children. This study tested the impact of type of cold pressor apparatus used (ice-vs. electric cooled) and different water temperature $\left(5^{\circ} \mathrm{C}, 7^{\circ} \mathrm{C}\right.$, and $\left.10^{\circ} \mathrm{C}\right)$ on pain-related outcomes in children.

METHODS: One hundred and sixteen healthy children $(57$ boys, 59 girls) aged 8 to 14 years $(\mathrm{M}=10.50$ years; $\mathrm{SD}=1.95)$ were randomly assigned to complete the CPTs with either an ice- $(n=55)$ or electric$(n=61)$ cooled apparatus. Children completed three consecutive CPTs with their non-dominant hand submersed in different water temperatures $\left(5^{\circ} \mathrm{C}, 7^{\circ} \mathrm{C}\right.$, and $\left.10^{\circ} \mathrm{C}\right)$ administered in counterbalanced order. Children rated pain intensity, pain affect, and pain-related fear for each CPT; pain tolerance times were recorded.

RESULTS: A series of 2 (apparatus: ice-vs. electric-cooled) $\times 3$ (water temperature: $5^{\circ} \mathrm{C}$ vs. $7^{\circ} \mathrm{C}$ vs. $10^{\circ} \mathrm{C}$ ) mixed analyses of variance (ANOVA) revealed significant main effects of water temperature on all of the pain outcomes, with the exception of pain affect; colder water temperatures were associated with more pain. There were no significant effects of apparatus on the majority of pain-related outcomes, with the exception of painrelated fear; children rated more fear when using the electric-cooled apparatus.

CONCLUSIONS: Use of different water temperatures for the CPT was associated with differential pain-related outcomes in children. Type of apparatus, however, generally did not impact pain-related outcomes. This information adds to our growing understanding of important variables to consider when administering the CPT to children. 
P4

THE REPORTING PRACTICES OF SEX AND GENDER IN PEDIATRIC STUDIES OF EXPERIMENTALLY INDUCED PAIN

Katelynn E Boerner ${ }^{1,2}$; Kathryn A Birnie ${ }^{1,2}$; Line Caes ${ }^{2}$;

Meghan Schinkel ${ }^{1,2}$; Christine T Chambers ${ }^{2,3}$

${ }^{1}$ Department of Psychology, Dalhousie University; ${ }^{2}$ Centre for Pediatric Pain Research, IWK Health Centre; ${ }^{3}$ Departments of Psychology and Pediatrics, Dalhousie University, Halifax, Nova Scotia

AIM: Appropriate reporting of sex (ie, biological differences) and gender (ie, socially constructed roles) in pain research is critical for advancing our understanding of the role of these variables in pain The aim of this systematic review was to examine reporting practices of sex and gender variables in pediatric studies of experimentally induced pain in healthy children METHODS: A search was conducted of key electronic databases (PsycINFO, EMBASE, CINAHL, PubMed). Eligibility criteria included: (1) empirical investigations that used an experimental pain task to examine pain-related outcomes; (2) English peer-reviewed manuscript; (3) community/healthy samples or a healthy control group of children between 0 and 18 years; (4) samples included both boys and girls. Papers were coded based on reporting practices of pain outcomes related to sex and gender. RESULTS: The search yielded 519 abstracts, with 76 separate studies eligible for inclusion. Fewer than half of included studies used the terms "sex" and/or "gender" appropriately; $36.8 \%$ of studies used "gender" when grouping children based on sex, 9.2\% of studies used "sex" and "gender" interchangeably, and $10.5 \%$ of studies did not use the terms "sex" or "gender" at all. $46.1 \%$ of studies reported statistical tests examining sex differences for at least one of their pain-related outcomes.

CONCLUSIONS: Use of sex and gender terminology was often inappropriate, and only half of studies reported tests of sex or gender differences in pain-related outcomes. Researchers need to demonstrate better awareness of the important distinctions between sex and gender in their pediatric pain research studies and papers.

\section{P5}

OWN WORRIES AND STRESS LEVEL INFLUENCE HEALTH CARE PROFESSIONAL AND PARENTS' ESTIMATION OF CHILD PAIN DURING A PAINFUL MEDICAL PROCEDURE

Line Caes ${ }^{1}$; Tine Vervoort ${ }^{2}$; Patricia Devos ${ }^{3}$; Joris Verlooy ${ }^{3}$; Yves Benoit $^{2,3}$; Christine Chambers ${ }^{1}$; Liesbet Goubert ${ }^{2}$

${ }^{1}$ Centre for Pediatric Pain Research, IWK Health Centre, Halifax, Nova Scotia; ${ }^{2}$ Ghent University, Department of ExperimentalClinical and Health Psychology; ${ }^{3}$ University Hospital Ghent, Ghent, Belgium

AIM: Children with leukemia recurrently undergo painful medical procedures such as lumbar punctures (LP) and bone marrow aspirations (BMA). As children are highly dependent on others for pain control, accurate pain estimation by caregivers has important implications for treatment. Despite research interest in pain estimation and its clinical importance, little is known about the mechanisms underlying observers' estimations of child pain. The purpose of the current study was to investigate the impact of parents' and different health care provider's LP/BMA-related worries and stress level upon estimation of child pain.

METHODS: Participants were 31 children recently diagnosed with acute lymphoblastic or myelogenous leukemia ( $55 \%$ boys, $\mathrm{M}$ age $=6.53$ years). The physician who performed the painful procedure, the assisting nurse, the occupational therapist and the parent accompanying the child were, before each LP/BMA procedure, requested to report on their worries regarding the child's pain. Afterwards, they were requested to report on their experienced level of stress and estimate the child's experienced pain intensity. Children older than 4 years were also requested to report on their experienced pain.

RESULTS: Multilevel analyses indicated that heightened levels of worry and stress are related to higher pain estimates by parents and all different health care providers. Additionally, for some, but not all different health care providers worry and/or stress was also related to more accurate pain estimations.

CONCLUSIONS: The current findings suggest that observers' emotional experience of painful medical procedures might have an important impact on their pain estimates, which might in turn influence management of the child's pain.
P6

EVIDENCE-BASED DEVELOPMENT AND INITIAL VALIDATION OF THE PAIN ASSESSMENT CHECKLIST FOR SENIORS WITH LIMITED ABILITY TO COMMUNICATE-II (PACSLAC-II)

$\underline{\text { Sarah Chan }}^{1}$; Thomas Hadjistavropoulos ${ }^{2}$

${ }^{1}$ Health Psychology Laboratory; ${ }^{2}$ Department of Psychology and Centre on Aging and Health, University of Regina, Regina,

Saskatchewan

AIM: The 60-item Pain Assessment Checklist for Seniors with Limited Ability to Communicate (PACSLAC) has been shown to be one of the most psychometrically and clinically sound methodologies for assessing pain in persons with dementia. Moreover, the PACSLAC is the only tool of its kind that comprehensively covers all pain assessment domains recommended by the American Geriatrics Society (AGS). We aimed to develop and validate a revised PACSLAC of shorter length containing items that are more pain-specific and covering all AGS-recommended domains.

METHODS: The PACSLAC was revised based on the pre-existing empirical and theoretical literature with emphasis on reducing the length and selecting items most likely to discriminate pain states from other types of distress. The resulting 31-item PACSLAC-II was used to assess pain in persons with dementia undergoing painful procedures as part of routine care. We also examined the ability of the PACSLAC-II to differentiate among pain-related situations and baseline relative to other pain assessment tools for seniors with dementia.

RESULTS: The PACSLAC-II demonstrated satisfactory reliability and excellent validity. It discriminated across pain-related situations and baseline while accounting for more variance than any of the six other tools evaluated, including the original PACSLAC. Further the PACSLAC-II accounted for unique variance with respect to ability to discriminate across pain-related situations and baseline, even after controlling for variance accounted for by all other measures combined.

CONCLUSIONS: We developed the PACSLAC-II, a shorter version of the PACSLAC and demonstrated that, compared to pre-existing tools, it has superior ability to discriminate painful from non-painful states.

\section{P7}

\section{SUFFERING IN ADOLESCENTS WITH CANCER}

Christelle Khadra $^{1,2}$; Sylvie Le May ${ }^{1,2}$; Jacinthe Lachance Fiola ${ }^{1,2}$; Isabelle Tremblay ${ }^{3}$; France Dupuis ${ }^{1,2}$; Chantal Cara ${ }^{1}$; Geneviève Mercier ${ }^{4}$; Marie-France Vachon ${ }^{4}$

${ }^{1}$ Faculty of Nursing, University of Montreal; ${ }^{2} \mathrm{CHU}$ Ste Justine's Research Centre; ${ }^{3}$ Psychology Department, CHU Ste Justine's; ${ }^{4}$ Hemato-oncology Department, CHU Ste Justine's, Montreal, Quebec

AIM: The purpose of this study was to explore the concept of suffering and its components in adolescents with cancer.

METHODS: Semi-structured interviews were conducted with 19 adolescent patients and 16 healthcare professionals involved in the care of these patients. Qualitative content analysis was conducted on the transcribed verbatim of the interviews and a qualitative data analysis (QDA) software, MAXQDA 10 plus was used to assist with coding. The same process was repeated by an external reviewer followed by a peer debriefing to compare results.

RESULTS: Analysis of the verbatim yielded various emergent themes related to suffering: physical, psychological, spiritual, social, cognitive and global suffering. Subthemes were also identified for each principal theme. CONCLUSIONS: Adolescents with cancer face a double challenge related to their pivotal developmental age on one hand and to the cancer diagnosis on the other hand which doubles their suffering. Thus, it is crucial that healthcare professionals consider this aspect while taking care of these patients. A holistic and global approach of care would probably help to alleviate suffering in adolescents with cancer. 
P8

\section{A YOGA INTERVENTION FOR PAIN MANAGEMENT AND WELL-BEING IN INDIVIDUALS WITH SPINAL CORD INJURY: A DESCRIPTIVE PILOT STUDY}

Kathryn Curtis ${ }^{1}$; Claire Wicks ${ }^{2}$; Nicole Leong ${ }^{3}$; Nicole Saunders ${ }^{3}$; Joel Katz ${ }^{4}$; Sander L Hitzig ${ }^{3}$

${ }^{1}$ York University; ${ }^{2}$ Centre for Addictions and Mental Health;

${ }^{3}$ Toronto Rehabilitation Institute; ${ }^{4}$ York University, Toronto General Hospital, Toronto, Ontario

AIM: Yoga is a promising intervention for improving well-being and reducing pain in individuals with spinal cord injury (SCI) A qualitative approach was undertaken to better understand the potential benefits of a specialized yoga program for individuals with SCI

METHODS: After REB approval and informed consent, participants $(\mathrm{N}=6)$ took part in a weekly $4 \mathrm{~min}$ to $60 \mathrm{~min}$ yoga class for 8 weeks. Postintervention, participants underwent an interview to obtain their perceptions on the benefits of the program, and to gain feedback on its implementation. Interviews were analyzed using qualitative description and code-recode was conducted by two analysts to verify content validity of identified themes.

RESULTS: The themes for feedback were: 1) expectations; 2) program benefits; and 3) self-growth. Participants reported they expected the program to provide them with a novel experience of self $(n=3)$, activity engagement $(n=3)$, stress relief $(n=5)$, social support $(n=1)$, and pain relief $(n=1)$. Program benefits included present-mindedness $(n=4)$, freedom from regular experience $(n=3)$, physical awareness $(n=4)$, relaxation $(n=4)$, investing in the self $(n=2)$, and environmental factors $(n=2)$. Selfgrowth included increased mindfulness $(n=4)$, taking the practice to life $(n=2)$, physical changes $(n=2)$, relaxation $(n=3)$, achievement $(n=2)$ and pain relief $(n=2)$. Concerning program implementation, participants wanted more classes $(n=4)$, variety $(n=3)$, specialization $(n=2)$, commitment from other participants $(n=2)$ and fewer distractions $(n=3)$. Overall, participants were satisfied with the program $(n=5)$.

CONCLUSIONS: A tailored yoga program for individuals with SCI is well received and has the potential for promoting self-growth and reducing pain.

\section{P9}

\section{AUTOMATICITY OF OBSERVATIONAL PAIN CUES FOR CHILDREN AGED 4 TO 12}

SR Fashler ${ }^{1}$; K Sekhon ${ }^{2}$; J Versloot ${ }^{3}$; KD Craig ${ }^{2}$

${ }^{1}$ Department of Psychology, York University, Toronto, Ontario;

${ }^{2}$ Department of Psychology, University of British Columbia,

Vancouver, British Columbia; ${ }^{3}$ Li Ka Shing Knowlege Institute, St Michael's Hospital, Toronto, ON

AIM: Certain pain behaviors appear automatic or reflexive, whereas others present as voluntarily controlled, thereby influencing judgments of observers as to the reason for pain expression, pain severity, and its authenticity. This project examined whether this distinction would be evident within a comprehensive list of those pain cues used in observational pain measures for children aged 4-12. Research participants rated behavioral cues for the apparent degree of automaticity.

METHODS: A systematic literature search was conducted using MEDLINE, PsycINFO, and Web of Science, applying criteria restricting the search to empirical studies providing for development of children's observational pain assessment tools. Twenty-one articles satisfied criteria. The pain behaviours to be observed were extracted from each scale. All items were compiled onto one list, with duplicate and redundant items eliminated, yielding a set of 66 cues. Research participants were 277 individuals recruited through an online survey site. They viewed the list of cues and rated each on multiple scales associated with the automaticity/controlled dimension.

RESULTS: An exploratory factor analysis yielded three major factors, consistent with theoretical expectations. The "automatic" factor consisted of items related to facial expression, paralinguistics, and consolability. The items in the "controlled" factor were related to intentional movements, verbalizations, and social actions. "Ambiguous" factor items reflected more voluntary facial expressions, with judgment apparently relying more on context.

CONCLUSIONS: Pain behaviors in observational pain scales for children can be characterized as being automatic, controlled, and ambiguous, supporting a dual-processing model of pain expression.

\section{P10}

\section{THE EFFECTS OF ANXIETY SENSITIVITY, ILLNESS SENSITIVITY, AND FEAR OF PAIN ON PAIN CATASTROPHIZING ARE MEDIATED BY SENSITIVITY TO PAIN TRAUMATIZATION IN AN UNDERGRADUATE STUDENT SAMPLE WITH PAIN}

SR Fashler; V Kleiman; MG Pagé; KM Roosen; J Katz

Department of Psychology, York University, Toronto, Ontario

AIM: Pain catastrophizing is a central variable in the fear-avoidance model, contributing to the development of chronic pain. Previous research has found that anxiety sensitivity (AS), illness sensitivity (IS), and fear of pain (FP) are significant predictors of pain-catastrophizing (PC). We recently identified a factor, sensitivity to pain traumatization (SPT), that resembles the somatic, psychological/emotional, and behavioural features of a traumatic stress reaction to pain. The aim of the present study was to evaluate the relationships among AS, IS, FP, and SPT, and to examine whether the effects of AS, IS, and FP on pain catastrophizing (PC) are mediated by SPT.

METHODS: We recruited 180 students (mean age $=20.5, \mathrm{SD}=4.5,72.8 \%$ female) reporting ongoing pain on a daily $(42.8 \%)$, weekly $(39.4 \%)$, or monthly $(17.8 \%)$ basis. Participants completed online questionnaires measuring self-reported AS, IS, FP, PC, and SPT online for course credit. RESULTS: A bootstrapping ( $\mathrm{n}=5,000$ resamples) mediation analysis (Hayes, A.F. SPSS MEDIATE Macro Syntax Reference) with three IVs showed that the omnibus effect on PC was mediated significantly by SPT ( $\mathrm{R}$ squared $=0.57, \mathrm{p}<0.001)$. Mediation was complete for AS $(95 \% \mathrm{CI}$ 0.024 to 0.182 ) and FP ( $95 \%$ CI 0.002 to 0.102 ), and partial for IS (95\% CI 0.114 to 0.363 ).

CONCLUSIONS: The results suggest that the three putative risk factors for chronic pain exert their effects at least partially on PC through SPT. SPT may be a vulnerability factor for heightened pain catastrophizing among young adults with ongoing pain.

\section{P11}

RELATIONSHIP BETWEEN PERCEIVED INJUSTICE AND HEAT-PAIN THRESHOLDS, PAIN, AND PSYCHOLOGICAL FUNCTIONING IN INDIVIDUALS FOLLOWING A MOTOR VEHICLE COLLISION

Mary Friesen ${ }^{1}$; Allan Walton ${ }^{1}$; Joel Katz ${ }^{2}$

${ }^{1}$ York University; ${ }^{2}$ Department of Psychology and School of Kinesiology and Health Science, York University, Toronto, Ontario AIM: Perceived injustice is associated with increased pain and disability The aim of the present study was to examine perceived injustice as it relates to heat-pain thresholds, pain, and psychological variables in individuals who sustained motor vehicle collision (MVC) injuries

METHODS: After obtaining ethics approval and informed consent, 23 adults (mean age 43.3 years, 13 male, 10 female) with MVC injuries were recruited. Participants completed the following: Pain Disability Index (PDI); PTSD Checklist (PCL-C); Injustice Experience Questionnaire (IEQ); Pain Catastrophizing Scale (PCS); Beck Depression Inventory-II (BDI-II); Beck Anxiety Inventory (BAI); Clinical Anger Scale (CAS), Short-Form McGill Pain Questionnaire-2 (SF-MPQ-2). Heat-pain thresholds were obtained using a thermal stimulator.

RESULTS: Two groups were formed based on median IEQ scores $(\mathrm{Mdn}=40)$. Comparisons between high $(\mathrm{n}=12)$ and low $(\mathrm{n}=11)$ IEQ groups showed the high IEQ group had significantly higher scores on the PCL-C, PCS, BAI, CAS, and SF-MPQ-2. IEQ scores $(n=23)$ correlated significantly with the BDI-II, PCL-C, CAS, and PCS but not the PDI, BAI, SF-MPQ-2, or heat-pain thresholds.

CONCLUSIONS: In contrast to previous research, a relationship was not found between perceived injustice and pain disability. The cross-sectional nature of the study precludes knowing whether perceived injustice is a risk factor for, or an outcome of, higher levels of anger, pain catastrophizing, depressive symptoms, or post-traumatic stress symptoms. Prospective studies are required to determine the status of perceived injustice as a risk factor for poor outcome following a MVC. 
P12

EXPLORING THE FACTOR STRUCTURE OF ANXIETYRELATED CONSTRUCTS IN A PEDIATRIC SAMPLE WITH AND WITHOUT A HISTORY OF PERSISTENT PAIN

Samantha Fuss; Gabrielle Pagé; Joel Katz

York University, Toronto, Ontario

AIM: A number of anxiety-related constructs are believed to play a role in the development and experience of chronic pain. The purpose of this analysis is to compare the factor structure of pain anxiety, pain catastrophizing, anxiety sensitivity and general anxiety between those with and without a history of persistent pain in a community sample of children and adolescents.

METHODS: Following REB approval and participant consent/assent, the Child Anxiety Sensitivity Index (CASI; physical, mental incapacitation, social and control), Children Pain Anxiety Symptoms Scale (CPASS; avoidance, fearful thinking, cognitive, and physiological responses), Pain Catastrophizing Scale - Child (PCSC; rumination, magnification, and helplessness) and Multidimensional Anxiety Scale for Children-10 (MASC-10) were completed by 1006 English-speaking individuals (54\% female, mean age $=11.6, \mathrm{SD}=2.7$ ) recruited to participate in this study, which took place at the Ontario Science Centre (Toronto, Canada). Twenty-seven percent of the sample $(n=269)$ reported having had pain that lasted for three months or longer. Principal Axis Factoring with Oblimin rotation was used to explore the underlying structure of the measures using their subscales between those with and without a history of persistent pain.

RESULTS: A two-factor solution best fit the data in both those with and without a history of persistent pain. The first factor, consisting of the subscales of the CPASS, PCSC and MASC-10, had Eigenvalues of 4.7 and 4.5 (accounting for $39.1 \%$ and $37.2 \%$ of the variance in those with and without a history of persistent pain), respectively. The subscales of the CASI comprised the second factor, with Eigenvalues of 2.4 and 2.4 (accounting for $19.6 \%$ and $18.9 \%$ of the variance between those with and without a history of pain), respectively.

CONCLUSIONS: This analysis demonstrates that the factor structure underlying these constructs is similar in both those with and without a history of persistent pain. In particular, it appears that anxiety sensitivity is distinct from pain-specific anxiety and general anxiety among children and adolescents regardless of their pain history.

\section{P13}

DEVELOPMENT AND MIXED-METHODS EVALUATION OF A PAIN ASSESSMENT VIDEO TRAINING PROGRAM FOR LONG-TERM CARE STAFF

Michelle M Gagnon; Thomas Hadjistavropoulos; Jaime Williams

Department of Psychology, University of Regina, Regina,

Saskatchewan

AIM: Inadequacies of pain assessment and management in long-term care (LTC) have been well documented. Insufficient pain education and inaccurate beliefs about the nature of pain and aging have been identified as possible contributors to this problem. This study addresses the need for improved continuing pain education through the development and evaluation of a training video on pain assessment in LTC.

METHODS: A total of 148 LTC staff watched and evaluated the training video. Knowledge was assessed pre-video, post-video and at a four week follow up. Beliefs about pain, as well as pain and aging, were also examined (using multivariate procedures) to determine whether these variables influenced participants' evaluation of the video. Focus groups were also conducted, and transcripts were analyzed using thematic content analysis. RESULTS: Pain assessment knowledge improved post video and at the four-week follow-up. Participants positively evaluated the video's content and quality. Individuals who held stronger beliefs (at baseline) about the organic nature of pain provided more positive evaluations. Barriers to implementation of practices in the video identified through the focus groups (and qualitative analysis) included time, workload, and resistance to change. Facilitators to implementation included continued management support and seeing the benefits to implementation.

CONCLUSIONS: This study provides support for the use of the training video. However, based on the focus group results, top-down implementation approaches with ongoing management involvement throughout the implementation process may be needed to achieve sustained changes in pain assessment practices. A model useful for sustained implementation was developed and discussed.

\section{P14}

INTERPRETATION BIAS OF NEGATIVE AND POSITIVE EMOTIONS IN PARTICIPANTS EXPERIENCING TONIC THERMAL PAIN

Wiebke Gandhi; Rebecca Price; Susanne Becker;

Petra Schweinhardt

The Alan Edwards Centre for Research on Pain, McGill University, Montreal, Quebec

AIM: The aim of this study was to investigate whether experimentallyinduced tonic pain leads to a cognitive-emotional bias causing altered interpretations of negative and positive emotions.

METHODS: Forty-five healthy participants were tested. In half $(n=22$; 12 females; mean age $23.8 \pm 7.0$ years), heat pain was continuously provoked while participants were involved in an emotional task. The other half served as control group ( $n=23 ; 12$ females; mean age 23.8 \pm 7.1 years). Controls experienced non-painful warm stimulation whilst performing the same task as the pain group. In this task computerized faces slowly changed from a neutral to an emotional expression (anger, disgust, fear, happiness). The participants' task was two-fold: 1) to stop the sequence at the first time point when the facial expression did not appear neutral anymore and 2) to indicate the emotion of the face at this time point.

RESULTS: The time point when participants stopped the sequence did not differ between groups. For the recognition of the emotion, however, the between-group comparison revealed that the pain group identified significantly more faces as disgusted $(\mathrm{p}=0.02)$ and significantly less faces as happy $(\mathrm{p}=0.04)$. Further, more perceived unpleasantness during the thermal stimulation was related to higher frequencies of indicating disgust $(r=-0.26, p=0.05)$ and to lower frequencies of indicating happiness (statistical trend, $r=0.22, p=0.07$ ).

CONCLUSIONS: Tonic pain induced a bias to interpret ambiguous emotional faces as more disgusted and less happy. Based on the notion of a moodcongruent cognitive bias (Wattkins et al., 1992), we conclude that tonic pain induced a negative emotional shift towards disgusted and less happy. FOOTNOTES/REFERENCES: Watkins PC, Mathews A, Williamson DA, Fuller RD (1992) Mood-Congruent Memory in Depression - Emotional Priming or Elaboration. J Abnorm Psychol 101:581-586.

\section{P15}

THE RELATIONSHIP OF PAIN ANXIETY AND LONG-TERM CARE STAFF PAIN BELIEFS

Omeed O Ghandehari; Thomas Hadjistavropoulos;

Amy JD Hampton; Jaime Williams

University of Regina, Regina, Saskatchewan

AIM: Within a fear-avoidance context, maladaptive beliefs about pain relate to activation of fear/anxiety, impacting upon behaviours (e.g., avoidance), coping methods, and disability. In long-term care (LTC) settings where chronic pain is common, clinicians have considerable influence over the activity levels of their patients, as well as treatment provision when pain is observed. However, few studies have investigated the psychological constructs related to pain in health care providers, which may influence their care provision. To this end, we aimed to determine whether a relationship exists between clinicians' beliefs about pain and their painrelated fear/anxiety responses.

METHODS: The Pain Anxiety Symptoms Scale Short form (PASS-20) and the Pain Beliefs Questionnaire (PBQ) including the aging beliefs subscale, added by Gagliese and Melzack (1997), were administered, as part of a larger study, to 110 LTC staff that regularly care for older adults living with chronic pain. Regression analyses were conducted to determine whether a relationship exists between specific types of pain beliefs and pain fear/anxiety in LTC staff.

RESULTS: Results indicated that stronger beliefs about the impact of organic pathology on the pain experience explained significant variance in the PASS-20 total scores beyond the contribution of demographic covariates. Beliefs about psychological factors in pain and beliefs concerning the relationship between pain and aging did not contribute significantly to the prediction. 
CONCLUSIONS: Results demonstrated that having increased organic pain beliefs may be a contributing factor to elevated pain-related anxiety in health care professionals. The implication of the findings for programs focusing on health professional education will be discussed.

\section{P16 \\ CONDITIONED PAIN MODULATION (CPM) TRIGGERED BY THE AFFECTIVE COMPONENT OF PAIN \\ Véronique Gougeon ${ }^{1}$; Isabelle Gaumond ${ }^{1}$; Philippe Goffaux ${ }^{1}$; Stéphane Potvin ${ }^{2}$; Serge Marchand ${ }^{1}$ \\ ${ }^{1}$ Université de Sherbrooke, Faculté de Médecine, Centre de recherche clinique Étienne-Le Bel du CHUS, Sherbrooke, Quebec; 2Université de Montréal, Centre de recherche Fernand-Seguin, Montreal, Quebec}

AIM: It has been demonstrated that the affective component of pain influences endogenous mechanisms of pain modulation. Moreover, pain-related cortical areas are activated by observing someone else in pain. A question that remains is did endogenous pain modulation mechanisms are activated by observing other's pain experience?

The aim of this study was to verify if the observation of ourselves or a loved one in pain can activate conditioned pain modulation (CPM).

METHODS: Ten healthy couples participated in four experimental sessions (baseline, pain condition, self condition and spouse condition). CPM was measured by the application of a thermal stimulation before and after the cold pressure test $(2$ minutes $)$ at $7^{\circ} \mathrm{C}$ or $20^{\circ} \mathrm{C}$. The reaction to the cold pressure test at $7^{\circ} \mathrm{C}$ was filmed. Participants were then asked to observe their video and the video of their spouse. During the video watching, the subject was doing the same protocol but with his/her arm in a non painful $20^{\circ} \mathrm{C}$ water bath.

RESULTS: The efficiency of CPM (in the spouse condition) correlates positively with the empathic score $(\mathrm{r}=-0.663 ; \mathrm{p}=0.037)$ for women while it correlates positively with the unpleasantness of the video for men $(r=0.715 ; p=0.046)$. Moreover, $t$-test showed that men were good at evaluating the intensity and unpleasantness of their spouse, while women had the tendency to underestimate the pain of their spouse (intensity: $t=2.852$; $\mathrm{p}=0.0019$; unpleasantness: $\mathrm{t}=2.482 ; \mathrm{p}=0.035$ ).

CONCLUSIONS: The observation of a significant person in pain is triggering CPM. However, the mechanisms are different between men and women where empathy is a contributing factor in women while perceived unpleasantness of the situation is the main factor in men.

\section{P17}

\section{DIFFERENCES OF BELIEFS ABOUT CANCER PAIN AND ANALGESICS USE BETWEEN TAIWANESE LUNG AND COLORECTAL CANCER POPULATION}

Shu-Liu Guo ${ }^{1}$; Doris Howell ${ }^{1}$; Yung-Chuan Sung ${ }^{2}$;

Judy Watt-Watson ${ }^{1}$; Mary Jane Esplen ${ }^{3}$

${ }^{1}$ Lawrence S Bloomberg Faculty of Nursing, University of Toronto, Toronto, Ontario; ${ }^{2}$ College of Medicine, Fu Jen Catholic University, Division of Hematology-Oncology, Department of Medicine, Cathay General Hospital, Taiwan; ${ }^{3}$ Department of Psychiatry, Faculty of Medicine, University of Toronto, Toronto, Ontario

AIM: Differences on cancer-related pain beliefs were examined between lung and colorectal cancer patients for pain-related outcome.

METHODS: A descriptive, inferential cross-sectional method was used. Patients were recruited from outpatient oncology department in Taiwan. Participants: Eligible patients were, (1) adults (>18 years) who were diagnosed with lung or colorectal cancer, and (2) reported cancer-related pain. Measures: Pain-related outcomes were measured using the Brief Pain Inventory-Short Form. Pain and analgesic beliefs were measured with Chinese version of Illness perception questionnaire-Cancer pain (IPQ$\mathrm{CaCP}$ ) and Beliefs about Medication Questionnaire (BMQ).

RESULTS: A total of 116 outpatients participated, the mean age was 63.12(SD=11.7) years, $51.7 \%$ were men, and $51.7 \%$ were colorectal patients. Forty-four $(80.4 \%)$ were diagnosed as stage IV on lung cancer patients. Twenty-six (43.3\%) and twenty-eight (46.7\%) were diagnosed as stage III and stage IV, separately on colorectal cancer group. Lung cancer patients reported lower scores on the physical performance statue than colorectal cancer group $(\mathrm{t}=-2.958, \mathrm{p}=0.004)$. Determined the beliefs about cancer pain for all of participants, there were higher score on subscale of timeline-IPQ-CaCP, and subscale of consequence-IPQ-CaCP. There was no significant difference on those beliefs about cancer pain between two groups. Meanwhile, those beliefs about cancer pain positively associated with the pain intensity and pain interference $(\mathrm{p}<0.05)$. Patients' beliefs about personal control and treatment control inversely related to their pain intensity and pain interference $(\mathrm{p}<0.05)$. Patients with colorectal cancer reported higher scores on subscale of personal control and subscale of treatment control than lung cancer group $(\mathrm{p}=-2.519, \mathrm{p}<0.05$; $\mathrm{t}=-2.167, \mathrm{p}<0.05$; separately). The average benefit on necessity-concerns about pain medication was positive (Mean: 2.26 \pm 7.04 ). That means patients' necessity beliefs about analgesics were higher than their concerns about pain medication. There was no difference on this benefit between two groups ( $\mathrm{p}>0.05)$.

CONCLUSIONS: The results of this study showed that colorectal cancer patients reported higher control beliefs about cancer pain than those with lung cancer. Patients' negative beliefs about cancer pain positively related to pain-related outcomes, and were no difference between two groups. Meanwhile, patients' necessity beliefs about analgesics use were higher than their concerns either lung cancer patients or those with colorectal cancer. Conclusions drawn from this study provided the basis for a future pain education intervention study with Taiwanese patients having lung or colorectal cancer.

\section{P18}

\section{COGNITIVE BEHAVIOURAL PAIN MANAGEMENT WITH OLDER ADULTS: DEVELOPMENT AND EVALUATION OF A TRAINING VIDEO FOR THERAPISTS}

Amy Hampton; Thomas Hadjistavropoulos; Michelle Gagnon; Jaime Williams

Department of Psychology and Centre on Aging and Health, University of Regina, Regina, Saskatchewan

AIM: Within the context of a large-scale knowledge translation project on pain in older adults, our goal was to develop and evaluate a Cognitive Behavioural Therapy (CBT) training video on chronic pain management in this population. The video is available free of charge.

METHODS: The professionally edited video was developed based on the scientific literature and experience of a senior clinical psychologist with expertise in pain in older persons. Participants ( 24 graduate students and 4 faculty members) were recruited from a Canadian doctoral program in clinical psychology independent of the authors' institution. They were shown the video and asked to complete an evaluation questionnaire assessing content and technical quality. Subsequently, two of the participating faculty members were interviewed and nine graduate students participated in a focus group. A mixed methods approach was used wherein quantitative ratings were summarized and thematic analysis of focus group and interview data was conducted.

RESULTS: Examination of participants' quantitative responses indicated positive ratings of both the video's content and technical aspects. Qualitative analysis indicated that participants valued the advanced clinical techniques and prominent theories presented in the video. They considered the video to be primarily appropriate for graduate student audiences and for presentation within the context of an introduction to the topic. CONCLUSIONS: Given the limited pain management instruction in Canadian training programs, this video represents a useful tool for augmenting health professional curricula.

\section{P19}

PSYCHOLOGICAL DETERMINANTS OF EXPECTANCIES FOR RECOVERY IN WHIPLASH ASSOCIATED DISORDERS

Johanna M Harrison ${ }^{1}$; Zina Trost $^{2}$; Michael JL Sullivan ${ }^{3}$ ${ }^{1}$ Department of Psychology, McGill University, Montreal, Quebec; ${ }^{2}$ Department of Psychology, University of North Texas, Denton, Texas, USA; ${ }^{3}$ Departments of Psychology, Medicine and Neurology, McGill University, Montreal, Quebec

AIM: The aim of the present study was to prospectively examine patients' specific expectancies for recovery during a rehabilitation intervention for Whiplash Associated Disorders (WAD) in order to investigate the determinants of expectancies, and the influence of psychological factors on the change in expectancy measures. We were particularly interested in understanding how the changes in various psychological factors over the course 
of the rehabilitation program would influence the change in expectancies. METHODS: The study sample consisted of 153 individuals (108 women, 45 men) who had sustained whiplash injuries in rear-collision motor vehicle accidents. Participants were enrolled in a standardized 7-week multidisciplinary functional restoration rehabilitation programs aimed at fostering recovery from whiplash injury. Measures of pain severity, pain catastrophizing, depression, pain-related fear of movement, and expectancies for recovery were completed at the initial assessment, mid-way through the program, and during the last week of the program.

RESULTS: Prospective analyses revealed that consistent with previous research, patients' positive expectancies for return to work at admission were significantly correlated with return to work at the end of treatment. Similarly, expectancy at admission for resumption of social and recreational activities was significantly correlated with resumption of such activity at the end of treatment. Univariate analysis revealed that patient expectancies for decreased pain, improved sleep, and resumption of socialrecreational activities were related to their levels of pain catastrophizing (PCS), kinesiophobia (TSK), and depression (BDI-II). Further multivariate analysis revealed that only pain catastrophizing was independently predictive of the patients' expectancies for decreased pain at admission. Whereas depression and pain-related fear of movement accounted for unique portions of the variance in patients' expectancies for improved sleep and return to work. Additional analyses of the changes in these psychological factors over the course of the rehabilitation program revealed that they were predictive of the changes in the patients' expectancies over the same period.

CONCLUSIONS: Patients' expectancies for recovery from musculoskeletal pain conditions have been identified as important factors in recovery outcomes. However, the determinants of these patient expectancies have remained unclear. The findings of this study provide some evidence regarding the determinants of patient expectancies for recovery, and provide potential targets for interventions in the modification of patients' expectancies.

\section{P20}

\section{ROLE OF PERCUTANEOUS CERVICAL CORDOTOMY IN} THE TREATMENT OF MEDICALLY REFRACTORY PAIN

Albert M Isaacs ${ }^{1}$; Mohamed Aly ${ }^{2}$; Christopher R Honey ${ }^{2}$

${ }^{1}$ Faculty of Medicine; ${ }^{2}$ Division of Neurosurgery, University of British Columbia, Vancouver, British Columbia

AIM: According to the WHO's 3-step pain ladder, an effective cancer pain relief regimen should involve the administration of nonopioids as first line, followed by mild opioids, before the use of stronger opioids. Recently, there has been the use of intraspinal analgesia as the fourth step for cancer-pain relief. Nonetheless, the administration of drugs in the right dose at the right time may still be ineffective for $10 \%$ to $20 \%$ of patients with cancer pain. For these patients with medically refractory pain, surgical intervention may be appropriate. We present the role of percutaneous cervical cordotomy (PCC) in the management of unilateral nociceptive cancer-related pain. PCC involves the ablation of the lateral spinothalamic tract, the primary pain-transmitting pathway of the spinal cord. We present 4 case reports from Vancouver General Hospital to demonstrate the potential success of PCC in providing palliative analgesia for otherwise intractable cancer pain.

METHODS: The in-patient charts of 4 Vancouver General Hospital patients who had undergone PCC between 2004 and 2012 were reviewed. Both objective and subjective data were extracted from each chart. Objective data included: the patient's gender, duration of admission during which the PCC was performed, type of cancer with confirmed metastases, pre-admission comorbidities, pre- and post-PCC analgesics regimen and patient-specific pre- and post-PCC complications. Subjective data included: pre- and post-PCC description of the cancer pain by the patient, their quality of life and the patient-care challenges faced by their respective healthcare providers while in hospital.

RESULTS: Pre-PCC, the patients were on an average of 9 different analgesics with over half of those being opioids. In addition, each patient required at least 2 different types of neurologic medications as an adjunct to managing their pain. All the patients described their pain as 10/10, while on the drugs before surgery and reported $0 / 10$ pain post-PCC. Well over $50 \%$ of their medications were discontinued after surgery, with majority of the remaining doses reserved as "pro re nata" for breakthrough pain.
Each patient demonstrated a relatively dramatic improvement in quality of life after surgery.

CONCLUSIONS: While treatment of cancer pain with analgesic medications may be effective for the majority of patients, there are some patients whose pain progressively becomes intractable. For patients with medically intractable, unilateral nociceptive pain, our results indicate that percutaneous cervical cordotomy can alleviate their pain and improve their quality of life in the palliative care setting.

\section{P21}

\section{PRE-OPERATIVE ANXIETY AND INTRA-OPERATIVE} ANALGESIC REQUIREMENTS

\section{Valery Kleiman-; Hance Clarke ${ }^{2}$; Joel Katz ${ }^{1,2}$}

${ }^{1}$ York University; ${ }^{2}$ Department of Anesthesia and Pain

Management, Toronto General Hospital, Toronto, Ontario

AIM: Higher preoperative anxiety has been associated with greater post-operative analgesic requirements. To our knowledge, nothing is known about the effects of preoperative anxiety on intraoperative analgesic requirements. The aim was to investigate the relationship between specific preoperative pain-related anxiety variables and intraoperative analgesic requirements. METHODS: 577 patients scheduled for major surgery were recruited $\sim 10$ days before surgery and were administered: Anxiety Sensitivity Index, Fear of Pain Questionnaire, Pain Anxiety Symptoms Scale-20, Pain Catastrophizing Scale, Pain Disability Index (PDI), Posttraumatic Stress Disorder Checklist, and State-Trait Anxiety Inventory. History of pain, current pain and preoperative pain pressure thresholds (PPTs) were also obtained. Total intraoperative dose $(\mathrm{mg} / \mathrm{kg}$ ) of opioid analgesics (fentanyl, morphine, hydromorphone, and their combinations) was obtained from the patient's anesthetic record and converted to morphine equivalents. RESULTS: Intraoperative opioid dose did not correlate with a history of previous surgery, or preoperative PPTs. Total intraoperative opioids dose positively correlated with PDI $(\mathrm{r}=0.10, \mathrm{p}=0.03)$ and negatively with age $(\mathrm{r}=-0.11, \mathrm{p}=0.01)$. Regression analyses revealed that none of the preoperative pain-related measures predicted total intraoperative opioid dose. However, when analyzed separately, trait anxiety predicted total dose of morphine $(F[1,212]=17.07, \mathrm{p}=0.000, \mathrm{R} 2=0.08)$ and all pre-operative painrelated measures combined significantly explained $11.8 \%$ of the variance in the total morphine dose in the OR $(F[8,138]=3.44, \mathrm{p}=0.001)$.

CONCLUSIONS: While previous research confirmed the negative relationships between preoperative anxiety and postoperative analgesic requirements and recovery, the current results suggest that intraoperative management is also significantly affected by patient's preoperative emotional state.

\section{P22}

\section{SEX DIFFERENCES IN INFANT FACIAL EXPRESSIONS OF PAIN-RELATED AND NON-PAIN-RELATED DISTRESS} Sara Ahola Kohut ${ }^{1,2}$; Rebecca Pillai Riddell ${ }^{1,3}$; David Flora ${ }^{3}$; Harriet Oster $^{4}$

${ }^{1}$ Hospital for Sick Children, ${ }^{2}$ University of Toronto; ${ }^{3}$ York University, Toronto, Ontario; ${ }^{4}$ New York University, New York, New York, USA

AIM: To determine whether facial expressions differ by infant sex after a no-pain and acute pain stimulus.

METHODS: Facial expressions of 100 infants were videotaped before and after their 2-, 4-, 6-, and 12-month immunization appointments. Facial expressions were coded using BabyFACS for one minute after a non-painful distressing stimulus (doctor's touch) and a painful distressing stimulus (immunization). Facial expressions were categorized by emotional valence (positive, neutral, negative) after both stimuli. Negatively valenced facial expressions after the immunization were then further subcategorized by the most commonly occurring facial expressions.

RESULTS: Proportion of time spent over the first minute after the doctor's touch was calculated for each emotional valence. Proportion of time spent over the first minute after the immunization was calculated for each emotional valence and facial expression category (i.e., pain-related vs. non-pain-related). A set of Independent Mann-Whitney U Tests was conducted to compare proportions of infants within each of the three emotional valence categories and seven distress expression categories across infant sex. There were no significant differences in the proportion of time 
spent in positive, negative, and neutral facial expressions between boy and girl infants over the first year of life. Similarly, there were no significant differences in the proportion of time spent in different discrete negative facial expressions of distress after the immunization injection between boy and girl infants over the first year of life.

CONCLUSIONS: Infant facial expressions of pain-related and non-painrelated distress do not differ between boys and girls in the first year of life.

\section{P23}

\section{CLINICAL FEASIBILITY OF THE ICONIC PAIN ASSESSMENT TOOL FROM THE PERSPECTIVE OF ADOLESCENTS WITH CHRONIC PAIN AND THEIR MULTI-DISCIPLINARY TEAM OF HEALTHCARE PROVIDERS}

Chitra Lalloo ${ }^{1}$; Jennifer N Stinson 2,3,4; Stephen C Brown ${ }^{2,5}$; Fiona Campbell2,5; Lisa Isaac ${ }^{2,5}$; James L Henry ${ }^{6}$

${ }^{1}$ Medical Sciences Graduate Program, Faculty of Health Sciences, McMaster University, Hamilton, Ontario; ${ }^{2}$ Department of Anaesthesia and Pain Medicine; ${ }^{3}$ Department of Child Health Evaluative Sciences, The Hospital for Sick Children; ${ }^{4}$ Lawrence S Bloomberg Faculty of Nursing; ${ }^{5}$ Department of Anaesthesia, University of Toronto, Toronto; ${ }^{6}$ Department of Psychiatry and Behavioural Neuroscience, McMaster University, Hamilton, Ontario AIM: The Iconic Pain Assessment Tool (IPAT) is the first self-report tool that records pain quality, intensity, and location using a mixture of icons and word descriptors on a detailed virtual body-map. The aim of this study was to examine IPAT clinical feasibility from the perspective of adolescents with chronic pain and their team of multidisciplinary pediatric healthcare providers. The web-based IPAT was administered alongside a paper-based comparator tool, which was based upon the standard clinic interview questions typically used to assess pain.

METHODS: Adolescents diagnosed with chronic pain aged 12 to 18 used the IPAT and comparator tool (randomized order) to self-report pain before a scheduled clinic appointment, and then took part in a semistructured interview. The health-team used these pain reports (IPAT and comparator) during patient appointments, and subsequently took part in focus group interviews.

RESULTS: A total of 17 adolescents and 9 health-team members completed the study. All adolescents endorsed the IPAT as easy to use, easy to understand, and clinically useful for communicating with their healthteam. The median time required for completion of the IPAT and comparator tool was 3.3 minutes and 3.6 minutes, respectively. Members of the health-team described the tool as easy to interpret and useful for eliciting detailed sensory information about pain, and they identified surmountable barriers to future clinical implementation.

CONCLUSIONS: Interviews with adolescents and their health-team indicate that the IPAT (renamed the Pain QuILT) is a clinically feasible tool that may offer unique advantages over traditional assessment methods by empowering patients to better communicate and track their pain.

\section{P24}

SEX DIFFERENCES IN CHRONIC PAIN DESCRIPTION, SELFMANAGEMENT, AND SATISFACTION WITH ABILITY TO CONTROL PAIN.

Elizabeth Mann ${ }^{1}$; Elizabeth VanDenKerkhof ${ }^{1}$; Margaret B Harrison ${ }^{1}$; Sandra LeFort ${ }^{2}$

${ }^{1}$ Queen's University, School of Nursing, Kingston, Ontario; ${ }^{2}$ Memorial University, School of Nursing, St John's, Newfoundland and Labrador

AIM: To compare pain characteristics, medical and emotional self-management, and satisfaction with pain control between men and women.

METHODS: This study is a secondary analysis of a recent cross-sectional survey on chronic pain in the general population of Canada (VanDenKerkhof, et al, 2013). Respondents were screened for chronic pain with two questions: "Are you currently troubled by pain or discomfort, either all of the time or on and off?" and "Have you had this pain or discomfort for more than 3 months?" Respondents completed the Leeds Assessment of Neuropathic Symptoms and Signs Pain Scale, Level of Expressed Need scale, Brief COPE, body diagram, and rated satisfaction with ability to control pain.
RESULTS: Of the 740 participants reporting chronic pain, 375 were male $(51 \%)$ and 365 were female (49\%). Men and women described their pain in a similar way, however men were half as likely to report using or receiving one or more treatments/prescriptions for pain (relative risk $[R R]=0.5$ [ $95 \% \mathrm{CI} 0.3$ to 0.8$]$ ) and half as likely to report complete satisfaction with their ability to control pain ( $R R=0.5$ [95\% CI 0.3 to 0.7]). There was no difference between men and women in use of acceptance $\left(\chi^{2}=2.77\right.$, $\mathrm{p}=0.10)$ or denial $\left(\chi^{2}=2.02, \mathrm{p}=0.16\right)$ to manage the stress of living with pain.

CONCLUSIONS: Although pain characteristics are similar, men and women report different pain self-management experiences. Clinicians should consider these sex differences when discussing and recommending pain management options. Further research is needed to explore the interaction between sex and satisfaction with pain control.

FOOTNOTES/REFERENCES: VanDenKerkhof, E.G., Mann, E.G., Torrance, N, Smith, B., Bennet, M., Johnson, A., \& Gilron, I. (2013). The Epidemiology of Neuropathic Pain in Canada. Paper to be presented at Canadian Pain Society Conference, May 2013, Winnipeg, MB.

\section{P25}

PSYCHOLOGICAL MECHANISMS UNDERLYING

GENERALIZATION - A UNILATERAL DELAYED-ONSET MUSCLE SORENESS PARADIGM

Nils Georg Niederstrasser ${ }^{1,2}$; Michael Sullivan ${ }^{1}$; Johan Vlaeyen ${ }^{2}$ ${ }^{1}$ Department of Psychology, McGill University, Montreal, Quebec; ${ }^{2}$ Department of Psychology and Educational Sciences, KU Leuven, Flanders, Belgium

AIM: The primary objective of this study was to examine how fear of pain and movement affects participants' propensity to generalize estimated and experienced pain in a simple lifting task.

METHODS: A sample of 40 individuals participated in the study on two consecutive days. Participants completed the Tampa Scale of Kinesiophobia prior to testing. A delayed-onset muscle soreness (DOMS) induction paradigm was employed whereby soreness was induced unilaterally in the muscles of the upper trunk, arm, and shoulder. Estimated and actual pain ratings on a simple lifting task were compared prior to and following DOMS-induction.

RESULTS: Prior to the DOMS induction, participants rated the lifts on both sides as equally painful. After DOMS induction, participants rated their pain significantly higher on their ipsilateral side, while ratings on the contralateral side remained stable. Further analyses revealed a significant interaction between participants' ratings on their contralateral side and kinesiophobia. Participants high in kinesiophobia tended to increase in pain on their contralateral side, while participants low in kinesiophobia tended to decrease, following DOMS-induction.

CONCLUSIONS: A significant interaction between fear of movement and pain ratings on the contralateral side may be interpreted as a sign of the generalization of pain from the ipsilateral side. Furthermore, this interaction points to contribution of high levels of kinesiophobia in this process.

Implications of these findings regarding biopsychosocial models of pain and the advancement of rehabilitation programs for chronic pain conditions and disability are discussed.

\section{P26}

\section{THE INFLUENCE OF PRODROMAL SYMPTOMS ON ACUTE} CORONARY SYNDROME PAIN INTENSITY

Sheila O'Keefe-McCarthy ${ }^{1}$; Michael McGillion ${ }^{1}$; J Charles Victor ${ }^{1}$; Judith McFetridge-Durdle ${ }^{2}$

${ }^{1}$ University of Toronto, Toronto, Ontario; ${ }^{2}$ Memorial University, St John's, Newfoundland and Labrador

AIM: Increasing evidence suggests that both men and women exhibit non-traditional prodromal symptoms prior to a cardiac event. The subjective, variable nature of cardiac pain poses a challenge to health care professionals with respect to assessment, diagnosis and timely management of acute coronary syndrome (ACS) related-pain. This study examined the relationship of prodromal symptoms with ACS pain intensity.

METHODS: A descriptive-correlational design was used to identify prodromal predictors of ACS cardiac pain intensity. One hundred and twentyone adults (63 males) admitted to a rural community emergency 
department, diagnosed with ACS, completed a prodromal questionnaire that described pre-hospital admission prodromal symptoms and symptom frequency and intensity. Baseline cardiac pain intensity ratings were collected from ACS patients.

RESULTS: Overall, while controlling for age and sex, bivariate correlations and linear regression revealed that baseline ACS pain intensity was significantly associated with pre-hospital prodromal symptoms $F(1,110)=2.464$, $\mathrm{P}=0.17, \mathrm{r} 2=0.152$. In particular, headaches $\mathrm{F}(1,118)=11.898, \mathrm{P}=0.001$, r2 $=0.092$; sleep disturbance $F(1,117)=4.726, P=0.032, r 2=0.039$; and anxiety $F(1,118)=4.819, \mathrm{P}=0.030, \mathrm{r} 2=0.030$ accounted for $15 \%$ variance in cardiac pain severity.

CONCLUSIONS: These preliminary findings suggest that that prodromal symptoms, particularly headache, sleep disturbance, and anxiety have important implications for ACS pain intensity. This extends our current knowledge of the predictive models of prodromal symptoms which predict ACS-pain-related outcomes. A larger prognostic study is required in order to determine whether 1) prodromal symptoms are predictive of future cardiac events in men and women, and 2) prodromal symptoms are a more significant predictor of ACS pain than common comorbidities such as hypertension, diabetes, abdominal obesity, hyperlipidemia and smoking.

\section{P27}

\section{A 12-ITEM SENSITIVITY TO PAIN TRAUMATIZATION SCALE: PRELIMINARY FACTOR STRUCTURE AND VALIDATION}

MG Pagé; MR Escobar; V Kleiman; SR Fashler; KM Roosen; J Katz Department of Psychology, York University, Toronto, ON

AIM: We recently identified a construct termed sensitivity to pain traumatization (SPT) that resembles the somatic, psychological/emotional, and behavioural features of a traumatic stress reaction to pain (Kleiman et al. Pain Res Manag 2011;16:169-177). The present study evaluated the preliminary factor structure as well as the construct and discriminative validity of a 12-item SPT scale (SPTS).

METHODS: 580 undergraduate students (mean age $=19.6, \mathrm{SD}=3.2$ years, $69.2 \%$ female) were recruited. In order to assess the number of factors to retain, we used Velicer's MAP and parallel analysis (PA) procedures on the matrix of polychoric correlations.

RESULTS: Velicer's MAP, PA, the Kaiser rule (eigenvalue $>1$ ) and the ratio of the first (5.57) to the second (0.38) eigenvalue $(>4)$ suggested that the SPTS is unidimensional. In addition, the SPTS demonstrated good internal consistency $(\alpha=0.88)$. SPTS scores correlated significantly $(r=0.68$, $\mathrm{p}<0.001$ ) with scores on the PTSD Checklist-Civilian version. Participants reporting moderate to severe pain interference $(n=79)$ scored significantly higher on the SPTS than participants reporting no or low pain interference $(\mathrm{n}=179)(\mathrm{t}(226)=4.65, \mathrm{p}<0.001)$.

CONCLUSIONS: In a sample of undergraduate students, the SPTS measures a single construct. The high positive correlation between the SPTS and a measure of PTSD and the greater SPTS scores among participants with higher pain interference scores suggests that the SPTS has good preliminary construct and discriminative validity.

\section{P28}

TIME-DEPENDENT PSYCHOLOGICAL PREDICTORS OF PAIN INTENSITY AND UNPLEASANTNESS IN CHILDREN UP TO ONE YEAR AFTER PEDIATRIC MAJOR SURGERY: PAIN TRAJECTORIES FROM HOSPITAL TO HOME MG Pagé ${ }^{1}$; F Campbell ${ }^{2,3}$; J Stinson ${ }^{2,4}$; L Isaac ${ }^{2,3}$; SR Fashler ${ }^{1}$; J Katz ${ }^{1,4,5}$

${ }^{1}$ Department of Psychology, York University; ${ }^{2}$ Department of Anaesthesia and Pain Medicine, Hospital for Sick Children; ${ }^{3}$ Department of Anaesthesia, Faculty of Medicine, University of Toronto; ${ }^{4}$ Faculty of Nursing, University of Toronto; ${ }^{5}$ Department of Psychology, Hospital for Sick Children, Toronto, Ontario

AIM: The goal of this study was to identify pain-related psychological predictors of pain intensity and unpleasantness trajectories over the first year after pediatric surgery.

METHODS: Eighty-three children $($ male $=33 \%)$ between the ages of 8 and $18(\mathrm{M}=13.2, \mathrm{SD}=2.4)$ completed the Child Pain Anxiety Scale, the Pain Catastrophizing Scale for Children, and the Numerical Rating Scale for pain intensity and unpleasantness 48 to 72 hours, two weeks (except for pain catastrophizing), and six and twelve months after major surgery. RESULTS: Generalized Estimating Equations showed a significant 3-way interaction between asymptotic time (the time (since surgery) transformed using an asymptotic function to better characterize the pattern by which pain scores decrease over time), pain anxiety, and pain catastrophizing in predicting variability in pain intensity (Wald $\chi^{2}=5.20, \mathrm{p}=0.02$ ) and pain unpleasantness (Wald $\chi^{2}=5.74, p=0.02$ ) over time. More specifically, children who reported high levels of both pain anxiety and pain catastrophizing had initially higher levels of pain intensity and unpleasantness compared to children with lower levels of pain catastrophizing and/or pain anxiety. Additionally, the rate of decline in pain intensity and unpleasantness scores was slower for children with initially high levels of both pain anxiety and pain catastrophizing.

CONCLUSIONS: Pain catastrophizing and pain anxiety are important predictors of variability in pain intensity and unpleasantness scores over the first year after pediatric surgery. Further research is needed to examine their contribution to the development and maintenance of chronic postoperative pain.

\section{P29}

THE EFFECTS OF SWITCHING OPIOID ANALGESICS FROM OXYCONTIN TO OXYNEO ON PAIN MANAGEMENT IN ADULTS WITH CHRONIC PAIN

Denise Paneduro ${ }^{1}$; Marilyn Galonski²; Senthilkumar Raju ${ }^{2}$; Andrew J Smith ${ }^{2,3,4}$; Allan Gordon ${ }^{2,5}$

${ }^{1}$ Department of Psychology, York University; ${ }^{2}$ Wasser Pain Management Centre, Mount Sinai Hospital; ${ }^{3}$ Pain and Addiction Medicine, Centre for Addiction and Mental Health (CAMH), ${ }^{4}$ Division of Neurology, University of Toronto; ${ }^{5}$ University of Toronto, Toronto, Ontario

AIM: In an effort to deter substance misuse of OxyContin tablets, a reformulated controlled-release product (OxyNEO), intended to be more difficult to dissolve and crush, was introduced in August 2010. The primary aim of this project was to describe the pain management experience of chronic pain patients at the WASSER Pain Management Centre after the introduction of OxyNEO.

METHODS: Twenty chronic pain patient charts (Mean age $=49.45, \mathrm{SD}$ $=11.17$ ) were reviewed to assess pain management before and after the introduction of OxyNEO.

RESULTS: Four out of 20 patients (20\%) who switched from OxyContin to OxyNEO reported that the reformulated tablet was not helpful for them and were prescribed other medications. The reasons provided by patients included 1) OxyNEO was not effective for managing their pain, 2) OxyNeo ran out more quickly than OxyContin, and 3) OxyNEO caused chest problems and breathing difficulty. No patients reported withdrawal symptoms while on OxyContin, however, after switching to OxyNEO, 7 of the 20 patients $(35 \%)$ reported withdrawal symptoms. Moreover, the OxyContin dose amount prior to the switch was positively associated with the presence of withdrawal symptoms after patients switched to OxyNEO, $\mathrm{r}(18)=0.57, \mathrm{p}<0.01$. Patients who reported withdrawal symptoms while on OxyNEO also reported greater pain severity, $\mathrm{r}(16)=0.57, \mathrm{p}<0.05$.

CONCLUSIONS: Patients reported greater withdrawal symptoms and difficulty managing their pain when required to switch to OxyNEO. These findings emphasize the need to regularly examine patients' pain management experience, particularly when medications are adapted, in order to ensure optimal health care for chronic pain patients.

\section{P29A}

\section{FACTORIAL VALIDITY OF THE ENGLISH LANGUAGE VERSION OF THE PAIN CATASTROPHIZING SCALE - CHILD VERSION}

Holly A Parkerson ${ }^{1}$; Gordon JG Asmundson ${ }^{1}$; Melanie Noel ${ }^{2}$; Gabrielle M Pagé ${ }^{3}$; Samantha Fuss ${ }^{3}$; Joel Katz ${ }^{3}$

${ }^{1}$ University of Regina, Regina, Saskatchewan; ${ }^{2}$ Dalhousie University, Halifax, Nova Scotia; ${ }^{3}$ York University, Toronto, Ontario AIM: The Pain Catastrophizing Scale (PCS; Sullivan et al., 1995) was developed in English to assess three components of catastrophizing (rumination, magnification, helplessness). The PCS has since been validated in many different languages and adapted for use with Flemish-speaking 
children (PCS-C; Crombez et al., 2003) and French-speaking adolescents (PCS-ado; Trembelay et al., 2008). The PCS-C has been back-translated to English and used with English-speaking children. There have been no assessments of the factorial validity of the English PCS-C. This study assessed the factor structure of the English PCS-C.

METHODS: Participants included English-speaking children ( $n=1006$; 8 to 18 years of age) recruited from the community. Exploratory factor analysis (EFA) was conducted using a random subsample of children $(n=504)$ to assess the underlying factor structure of the PCS-C. Items with poor loadings on factors were removed from further analysis. Confirmatory factor analysis (CFA), using the second subsample of children $(n=502)$, was used to cross-validate the factor structure revealed by the EFA and compare it to the original 3 -factor model and other model variants.

RESULTS: EFA revealed that an oblique 3 -factor model representing 11 of the original 13 PCS-C items, all of which loaded on their original factors (rumination, magnification, helplessness), provided the best fit to the data. CFA indicated the 11-item, 3-factor model provided an adequate and superior fit to the data when compared to the original 3-factor model and other model variants.

CONCLUSIONS: The English PCS-C may be better understood using a revised 11-item oblique 3-factor model. Comprehensive results, limitations, and implications are discussed.

\section{P30}

\section{EFFECTS OF OPIOID ANTAGONISM ON PAIN SENSITIVITY IN OBESE PATIENTS AT HIGH OR LOW RISK OF OBSTRUCTIVE SLEEP APNEA}

Rebecca Price ${ }^{1,2}$; Juan Francisco Asenjo ${ }^{3,4}$; Nicolas V Christou ${ }^{5}$; Laura Stone $^{2,6}$; Steven B Backman ${ }^{7}$; Petra Schweinhardt ${ }^{1,2,6}$

${ }^{1}$ Faculty of Medicine; ${ }^{2}$ The Alan Edwards Center for Research on Pain, McGill University; ${ }^{3}$ McGill University Department of Anesthesia, Montreal General Hospital; ${ }^{4}$ The Alan Edwards Pain Management Clinic, McGill University; ${ }^{5}$ McGill University Department of Surgery, Royal Victoria Hospital; ${ }^{6}$ Faculty of Dentistry, McGill University; ${ }^{7}$ McGill University Department of Anesthesia, Royal Victoria Hospital, Montreal, Quebec

AIM: To assess whether obese patients at high risk for obstructive sleep apnea (OSA) are more sensitive to the effects of the opioid antagonist naltrexone on experimental pain.

METHODS: Using a double-blind placebo-controlled crossover design obese patients at high risk (HR) $(n=10)$ or low risk (LR) $(n=10)$ for OSA were compared to age- and gender-matched non-obese participants $(n=20)$. In each session, cold and heat pain thresholds and pain tolerance were assessed using the method of limits with a $16 \times 16 \mathrm{~mm}$ thermode (TSAII, Medoc, Israel) on the forehead and abdomen. Subjective pain intensity and unpleasantness ratings for tonic one-minute $\left(48^{\circ} \mathrm{C}\right)$ and for phasic 5 -second (threshold $+2^{\circ} \mathrm{C}$ or $+4^{\circ} \mathrm{C}$ ) stimuli were assessed on the forehead.

RESULTS: For forehead tonic $(\mathrm{p}=0.013)$ and phasic $(\mathrm{p}=0.071)$ heat intensity, pain sensitivity increased following naltrexone in LR obese and non-obese, but did not change in HR obese. For baseline sensitivity across sessions, LR obese were less pain sensitive for forehead heat pain tolerance $(p=0.089)$ and phasic heat pleasantness ratings $(p=0.009)$ than HR obese and non-obese who did not differ. However, for abdomen heat pain threshold $(p=0.021)$ and tonic cold intensity $(p=0.013)$, LR and HR obese did not differ and were less sensitive than non-obese. No drug effects were found for these measures.

CONCLUSIONS: Thus, it appears that HR OSA obese patients are less reactive to opioid antagonism than LR obese and non-obese patients. Moreover, except on areas of much excess subcutaneous fat, LR patients are less sensitive to pain than non-obese or HR obese patients.

\section{P31}

\section{THE INFLUENCE OF PSYCHOLOGICAL FACTORS ON THE RECALL OF CLINICAL AND EXPERIMENTAL PAIN}

Sylvia Roy; Marie-France Boisjoli; Joanie Gauthier; Geneviève Bélanger; Kathya Daigle; Guillaume Léonard; Philippe Goffaux

Université de Sherbrooke, Sherbrooke, Quebec

AIM: The long-term recall of past pain is altered compared to its initial experience, a phenomenon called mnemonic pain bias. In this study we wanted to know if the mnemonic bias of clinical and experimental pain were related, and, if psychological factors influenced the recall of clinical and experimental pain in a similar fashion.

METHODS: Seventeen patients suffering from upper-extremity musculoskeletal pain were recruited and exposed to a cold-pressor test for two minutes (i.e., water set at $12^{\circ} \mathrm{C}$ ). Both clinical and experimental pain intensity scores were assessed using a visual analogue scale (VAS) devoid of numerical anchors. Following a two month delay, participants were asked to recall the intensity of clinical and experimental pain at initial time-oftesting (T1). Signs and symptoms of depression, anxiety, and, pain catastrophizing were also assessed at T1.

RESULTS: Mnemonic bias of clinical and experimental pain were unrelated $(r=0.14, p=0.59)$. Hierarchical regression analysis conducted to identify the most reliable subset of recalled pain predictors showed that the recall of clinical pain depended on both T1 clinical pain intensity ratings and $T 1$ depression scores $(\mathrm{p}=0.05)$, whereas the recall of experimental pain depended only on T1 experimental pain intensity ratings $(\mathrm{p}=0.001)$.

CONCLUSIONS: Although the remembrance of pain is highly influenced by its initial experience, the presence of a depressed mood significantly magnified the long-term recall of clinical but not experimental pain.

\section{P31A}

\section{PRELIMINARY VALIDATION OF A FRENCH VERSION OF THE CHRONIC PAIN ACCEPTANCE QUESTIONNAIRE}

Whitney Scott; Elena Bernier; Rosalind Garland; Michael Sullivan McGill University, Montreal, Quebec

AIM: Emerging research suggests that pain acceptance may play an important role in adaptation to chronic pain. Acceptance of pain involves reducing unsuccessful attempts to avoid or control pain, and engaging in personally meaningful activity even when pain is experienced. The Chronic Pain Acceptance Questionnaire (CPAQ) is one tool that has been used to assess pain acceptance, and research supports its psychometric properties (McCracken et al., 2004). Despite translation of the CPAQ into several languages, a French translation has yet to be empirically validated. Thus, the purpose of this study was to examine the psychometric properties of a French version of the CPAQ.

METHODS: One hundred and fifty eight individuals with chronic musculoskeletal pain participated in this study. The CPAQ was translated to French, then back-translated to English using a standard translation procedure (Vallerand, 1989). Participants completed the translated CPAQ and self-report measures of pain intensity, disability, and cognitive-affective variables related to pain.

RESULTS: Chronbach's coefficient alpha suggested adequate internal consistency. Principle components analysis revealed a factor structure similar to the original CPAQ. Convergent validity was suggested by significant negative correlations between pain acceptance, depressive symptoms, state anxiety, and self-reported disability. Discriminant validity was suggested by the relative magnitude of the correlations between pain acceptance and pain intensity, and acceptance and self-reported mental health symptoms and disability.

CONCLUSIONS: These results provide initial support for the reliability and validity of a French translation of the Chronic Pain Acceptance Questionnaire

\section{P32}

REDUCTIONS IN PAIN CATASTROPHIZING FOLLOWING PARTICIPATION IN A PHYSICAL THERAPY INTERVENTION FOR MUSCULOSKELETAL INJURY

Peter Slepian; Elena Bernier; Whitney Scott;

Nils Georg Niederstrasser; Timothy Wideman; Michael Sullivan Department of Psychology, McGill University, Montreal, Quebec AIM: The aim of the present study was to investigate factors that contribute to the resistance of pain catastrophizing during the course of a physical therapy intervention for musculoskeletal injury.

METHODS: One hundred and eighty-seven patients completed selfreport measures of pain catastrophizing and mental health variables at the beginning and end of a 7-week physical therapy intervention. Using pretreatment assessment data, patients were divided into four mutually exclusive groups using previously established cut-offs on the Pain Catastrophizing 
Scale, Beck Depressive Inventory-II, and the Impact of Events Scale Revised. The proportion of patients in each of these pre-treatment assessment groups was compared with respect to catastrophizing treatment resistance, operationally defined as remaining above an established cutoff on catastrophizing following treatment. Treatment resistant and non-resistant patients also were compared according to clinicians' posttreatment judgments of return-to-work capacity.

RESULTS: High levels of pain catastrophizing and mental health symptoms were the most prevalent risk-profile (37\%), followed by low-risk (35\%), high catastrophizing alone (16\%), and mental health symptoms alone $(13 \%)$. Chi-square analyses demonstrated a significantly higher proportion of treatment resistant patients in the high catastrophizing and mental health symptom group than in any other group $(\mathrm{p}<0.001)$. Clinicians judged treatment resistant patients to be significantly less work-ready $(\mathrm{p}<0.05)$.

CONCLUSIONS: The high prevalence and number of treatment resistant patients presenting with both high levels of pain catastrophizing and mental health symptoms warrants their referral to specialized care. Physiotherapists may consider including elements of targeted psychosocial interventions into primary care to address high levels of catastrophizing.

\section{P33}

\section{PAIN ATTITUDES QUESTIONNAIRE: CORRELATES OF PAIN-RELATED STOICISM AND CAUTIOUSNESS IN PEOPLE WITH CANCER PAIN}

Kim Thao Tran ${ }^{1}$; Lynn R Gauthier ${ }^{2,3}$; Gary Rodin ${ }^{3,4}$;

Camilla Zimmermann ${ }^{3,4}$; David Warr ${ }^{3,4}$; S Lawrence Librach, 4 ; Malcolm Moore ${ }^{3,4}$; Frances A Shepherd ${ }^{3,4}$; Lucia Gagliese $e^{2,3,4,5}$

${ }^{1}$ University College Dublin School of Medicine and Medical Science, Dublin, Ireland; ${ }^{2}$ York University; ${ }^{3}$ University Health Network; ${ }^{4}$ University of Toronto; ${ }^{5}$ Mount Sinai Hospital, Toronto, Ontario

AIM: The revised Pain Attitudes Questionnaire (PAQR) is a valid measure of pain-related stoicism and cautiousness in people with chronic nonmalignant pain. This study aimed to validate it for patients with cancer pain and to identify the correlates of pain-related stoicism and cautiousness.

METHODS: 215 patients (mean age $56.9 \pm 11.7$ years; $57.2 \%$ female) with advanced cancer and pain, who attended outpatient clinics or received home palliative care, completed the PAQR and measures of pain and physical and psychosocial well-being. Demographic and clinical information was collected.

RESULTS: Principle components analysis determined that the best factor structure for the PAQR was a, two-subscale (Stoicism and Cautiousness) measure, the PAQ-Cancer (PAQ-C). Age, demographic factors and pain intensity, management and relief were not significantly correlated with the PAQ-C. Multiple regression analysis revealed that stoicism was associated with greater activity engagement $(\beta=0.425, \mathrm{p}<0.001)$, avoidant responses to a traumatic event $(\beta=0.300, p<0.001)$ and neuropathic pain qualities $(\beta=0.189, p<0.01)$. Cautiousness was associated with greater avoidant responses $(\beta=0.305, \mathrm{p}<0.001)$ and lower satisfaction with pain control $(\beta=-0.172, p<0.05)$.

CONCLUSIONS: The PAQ-C is a valid and reliable measure of painrelated stoicism and cautiousness in patients with advanced cancer. Our results do not support previous suggestions that older adults are more stoic than younger adults about pain. Instead, they suggest that stoicism and cautiousness may be related to some indices of pain and avoidant responses related to a traumatic event. Further research is needed to elucidate the roles of pain-related stoicism, cautiousness and responses to trauma in people with cancer pain.

\section{P34}

MEASURING "FRIENDSHIP" IN MICE USING A NOVEL PARADIGM: EFFECTS OF PAIN AND STRESS

Alexander Tuttle; Kimberly Dossett; David Yachnin; Philip Leger; Melissa Sukosd; Jean Sebastien Austin; Jeffrey Mogil McGill University, Montreal, Quebec

AIM: Pain-related empathy and social approach behaviours in mice require familiarity between animals. However, the social signals and corresponding neurobiological mechanisms mediating familiarity between animals, including pain behaviours, are currently unknown. Using a novel huddling paradigm, we were interested in characterizing how murine dyads of varying social status interact with one another, and how these interactions subsequently regulate glucocorticoid receptor (NR3C1) and corticotropin releasing hormone $(\mathrm{CRH})$ levels in relevant brain areas. Furthermore, we were interested in characterizing how acute and chronic pain subsequently influence murine social behavior and regulation of the HPA axis.

METHODS: Naïve, young adult (6 to 10 weeks old) outbred CD-1 mice were placed in familiar or strange, same-sex dyads at the start of testing. Socially familiar dyads were co-housed $>3$ weeks before start of testing. Social dyads were placed in an open field arena under a bright light $(250 \mathrm{~W})$ containing a single cylinder $(6 \times 3 \mathrm{~cm})$ placed against one wall. Mice were tested for 3 hours. Some stranger dyads received injections of MET ( $50 \mathrm{mg} / \mathrm{kg}$ ) $30 \mathrm{~min}$ before testing, all others received saline. Huddling behaviours in the cylinder were assessed offline using behavioural sampling. Relevant brain regions were also removed (central $\mathrm{n}$ amygdala, bed $\mathrm{n}$ stria terminalis, paraventricular $\mathrm{n}$ hypothalamus) and total RNA was isolated and reverse transcribed before further analysis.

RESULTS: Here we show that social (huddling) behaviour between stranger murine dyads increase over a brief exposure period ( $>3 \mathrm{~h}$ ), eventually becoming indistinguishable from cohabitating dyads. It appears that social status dictates early $(<1 \mathrm{~h})$ differences in the amount of active and passive social behaviours, and that these differences disappear over a longer period of exposure. Furthermore, by blocking corticosterone synthesis using the drug metyrapone (MET), we can completely reverse the initial stranger effect in both active and passive social paradigms. We also show that murine dyads of varying social status interact with one another differently when one animal is suffering from tonic inflammatory or chronic neuropathic pain. Our data suggest that levels of CRH mRNA in key brain structures involved with hypothalamic-pituitary-adrenal axis (HPA) activation are increased in unafflicted male stranger dyads when compared to those of male familiar dyads. We also show that interactions between animals of varying social status are correlated with different levels of GR mRNA expression.

CONCLUSIONS: Our findings suggest that familiarity in mice, upon which empathy and other pain-directed pro-social behaviors are based, is primarily dictated by the acute stress response. If familiarity in mice is solely regulated by stress, this research may better inform our understanding of the substrates of human relationships. Furthermore, by studying pain's effects on social behavior, our research is intended to illuminate how pain can serve as a social signal, significantly impacting both mammalian interpersonal behaviors and corresponding stress response.

\section{P35}

\section{DEVELOPMENT AND IMPLEMENTATION OF AN INTERDISCIPLINARY PAIN MANAGEMENT MODEL FOR OLDER ADULTS LIVING IN LONG-TERM CARE}

Haley Weber ${ }^{1,2}$; Ramesh Zacharias ${ }^{2,3}$, Carlos Rojas-Fernandez ${ }^{1,2}$

${ }^{1}$ University of Waterloo; ${ }^{2}$ Research Institute for Aging, Schlegel Senior Villages; ${ }^{3}$ McMaster University, Hamilton, Ontario

AIM: The purpose of this study is to develop a novel interdisciplinary pain management (IPM) model to better treat and manage pain within the elderly population residing in long-term care institutions.

METHODS: This project is being carried out as a multiphase study:

1. A medical record review (MRR) characterizing the usual care model currently relied upon in representative facilities. This will include review of 180 patient charts in representative facility.

2. One-on-one staff oriented interviews carried out with a variety of health-care professions to gather information regarding staff perceived barriers, challenges and strengths concerning current pain management practices. Grounded theory will be utilized to analyze transcripts and develop patterns.

3. Focus group session aimed at further exploring themes developed during one-on-one interviews. Discussion surrounding the development of framework for the new interdisciplinary model will also be included in this session.

4. Details of the interdisciplinary model will be delineated. This phase will encompass creation of all educational materials, tools, and standard operating procedures.

5. Implementation and trialing of the model will take place via comparison study. A cohort of residents will have pain scores measured before (usual 
care) and following implementation of interdisciplinary pain management model.

RESULTS: An interdisciplinary pain management model for patients in long-term care facilities is established. Implementation and trialing of the interdisciplinary model will prove to be more beneficial than the standard care model. Ultimately, this will be demonstrated by an overall improvement in resident pain scores.

CONCLUSIONS: The development and utilization of an interdisciplinary pain management model will provide a useful and efficacious method to treat pain in the aged living within long-term care facilities.

\section{P36}

\section{GREY MATTER CORRELATES OF FATIGUE IN ANKYLOSING} SPONDYLITIS PATIENTS

Qi Wu ${ }^{1}$; Robert Inman ${ }^{2,3}$; Karen D Davis ${ }^{1,4}$

${ }^{1}$ Division of Brain, Imaging \& Behaviour - Systems Neuroscience, Toronto Western Research Institute, Toronto Western Hospital; ${ }^{2}$ Division of Rheumatology, Toronto Western Hospital and Toronto Western Research Institute; ${ }^{3}$ Department of Medicine and Institute of Medical Science; ${ }^{4}$ Department of Surgery and Institute of Medical Science, University of Toronto, Toronto, Ontario

AIM: Fatigue is a common symptom that significantly impacts quality of life in many chronic pain conditions, including ankylosing spondylitis (AS) yet there is little known about the brain mechanism of fatigue. In this study we used structural MRI to study gray matter (GM) abnormalities related to fatigue in AS.

METHODS: Twenty patients with chronic back pain secondary to AS $(15 \mathrm{M} / 5 \mathrm{~F} ; 34.8 \pm 11.9$ years old $)$ and age/sex-matched controls consented to the approved study. Patients completed the Fatigue Severity Scale (FSS) and underwent 3 T MRI to acquire high resolution images. Brain GM was measured with cortical thickness analysis (CTA, Freesurfer) and voxel based morphology (FSL-VBM) for subcortical structures. Age and sex were included as covariates.

RESULTS: The AS patients had significant fatigue (FSS=6.8 \pm 2.4 ). Brain imaging analyses revealed that the GM of primary sensorimotor cortices (right S1 r=-0.79), bilateral M1 (left $\mathrm{r}=-0.81$; right $\mathrm{r}=-0.75$ ) and bilateral caudate nucleus (left $r=-0.51$; right $r=-0.59$ ) negatively correlated with fatigue $(\mathrm{P}<0.05)$, whereas there was a positive correlation $(\mathrm{P}<0.05)$ between GM of the left supplement motor areas (lateral cluster $r=0.80$; medial cluster $\mathrm{r}=0.69)$ and putamen $(\mathrm{r}=0.64)$, the right anterior insular $(\mathrm{r}=0.79)$ and dorsolateral prefrontal cortex $(\mathrm{r}=0.79)$, and thalamus $(\mathrm{r}=0.76)$.

CONCLUSIONS: The finding that fatigue severity in AS negatively correlates with GM in primary sensorimotor areas may reflect a role for sensory processing, mobility and flexibility in fatigue. However, the positive correlation of fatigue severity with GM in insula and prefrontal areas may relate to alterations in cognitive and affective functions in AS.

\section{P37}

\section{A SYSTEMATIC REVIEW OF THE LITERATURE ON MALINGERED CHRONIC PAIN \\ Leffrey H Ennis; Xinjie C Yin \\ McMaster University, Hamilton, Ontario}

AIM: The diagnosis of malingering has been used by clinicians to label individuals who intentionally produce false or grossly exaggerated physical or psychological for secondary gain. The purpose of this study is to review the literature on malingering to determine if it meets a reasonable standard as a psychiatric diagnosis that can be applied reliably.

METHODS: A review of the literature was conducted, from January 2000 until January 2013, using MEDLINE, EMBASE, PSYCHINFO, CINAHL, and CENTRAL. Examples of search terms include malingering and: symptoms of unknown etiology, secondary gain, scales (eg. TOMM), Carl Poper and falsifiability, physical examination, and patient credibility.

RESULTS: Over forty articles were reviewed. There is no evidence that the diagnosis of malingering is valid. Nor can the label be applied reliably. The construct does not meet Poper's criteria for falsifiability, and therefore it is not a scientific construct. There is evidence in the literature that many of the findings used to make the diagnosis of malingering are found in normal patient populations. These findings include inconsistency in verbal history, unusual and unexplained symptoms, and multiple Waddell signs.
CONCLUSIONS: Significant negative consequences are associated with the label of malingering. However, the evidence suggests that there are significant problems associated with making such a diagnosis. There is insufficient evidence that the label represents a valid diagnosis that can be reliably applied. Given the harm that can be caused my mislabeling a patient as malingering, it is important that criterion for this diagnostic entity be updated such that they meet Poper's criteria for falsifiability. REFERENCES:

1. DSM IV TR

2. Slick DJ, Sherman EM, Iverson GL. Diagnostic criteria for malingered neurocognitive dysfunction: proposed standards for clinical practice and research. Clin Neuropsychol 1999;13:545-61.

\section{P38}

EVALUATION OF THE IMPLEMENTATION AND IMPACT OF A PRIMARY CARE SUB-ACUTE LOW BACK PAIN PROGRAM: DEFINING KEY INDICATORS AND OUTCOMES

\section{Sara Ahmed $^{1}$; Regina Visca ${ }^{2}$; Roderick Finlayson ${ }^{3}$;}

Amédé Gogovor ${ }^{1}$; Mark Ware ${ }^{1}$

${ }^{1}$ McGill University; ${ }^{2}$ Centre of Expertise in Chronic Pain, RUIS

McGill; ${ }^{3}$ Montreal General Hospital, Centre of Expertise in Chronic Pain, Montreal, Quebec

AIM: Guided by evidence-based guidelines and the Chronic Care Model, our team has defined a clinical process for an interdisciplinary primary care and self-management program for patients suffering from sub-acute low back pain (SALBP) to be implemented in four social service networks (CSSS). The purpose of this abstract is to present the evaluation plan and indicators that were defined to evaluate 1) the impact of the program on patients' function and health-related quality of life, on primary healthcare practice, and healthcare utilization over a 2 year period, and 2) the extent to which each of the CSSS implement the various components of the clinical process and the contextual barriers and facilitators of implementation.

METHODS: We will use a mixed method approach to evaluate the impact of the program. This will include a quasi-experimental pretest/ posttest interrupted time series design and a quantitative evaluation of the effects across the 4 CSSS. Questionnaires will be administered to over 1200 patients and to primary care physicians. The qualitative component will be a multiple case study approach to determine the extent of implementation of the program in each CSSS.

RESULTS: To date, 2 CSSS have implemented the program. Twenty two patients reported and primary care indicators have been identified to evaluate the impact of the intervention.

CONCLUSIONS: The results from this study will allow us to provide recommendations for refining the program and knowledge transfer to other CSSS that will implement the SALBP clinical process, and inform the development of programs for other chronic pain patient populations.

\section{P39}

\section{UNEXPLAINABLE NON DERMATOMAL SOMATOSENSORY DEFICITS (NDSDS) IN CHRONIC PAIN PATIENTS REFERRED FOR A MEDICOLEGAL ASSESSMENT}

Maha Alkokani ${ }^{1}$; Nivan Zoheiry ${ }^{1}$; Angela Mailis-Gagnon ${ }^{1,2,3}$

${ }^{1}$ Comprehensive Pain Program, Toronto Western Hospital; ${ }^{2}$ Krembil Neuroscience Centre, Toronto Western Hospital, University of Toronto; ${ }^{3}$ Centre for the Study of Pain, Toronto, Ontario

AIM: To describe the characteristics of litigants with chronic pain and NDSD to multiple testing modalities (pinprick, light touch, cold, vibration sense and manual pressure)

METHODS: This is a cross-sectional descriptive study. Data were collected from of 33 consecutive litigants with NDSD referred for medicolegal consultation over 4 years. Demographics, pain drawings, pain ratings and maps of sensory abnormalities were collected at the time of intake while accident related and other details were obtained using retrospective chart review.

RESULTS: Litigants' mean age was 44 years (range 25 to 65 ) with 3 quarters been females; Canadian/ foreign born ratio was 2:1 for the males, and $1: 1$ for the females; highest education achieved was high school for $89 \%$ of the males and college/university for $42 \%$ of the females. Average pain duration was 3.3 years. Almost $90 \%$ of the males were labourers while $70 \%$ of 
the females were in professional/ office type jobs. Median pain rating for both sexes were 7/10 with females having higher scores than males. In $52 \%$ of the subjects, NDSDs had a hemisensory distribution. All subjects were deemed to have psychological factors involved in their presentation with $1 / 3$ with no detectable biomedical pathology. Circumstances of the injury and other characteristics of the litigants will be described in further analysis

CONCLUSIONS: NDSDs have been shown to be associated with treatment resistant pain, poor prognosis for return to employment, and brain activation changes attributed to "maladaptive neuroplasticity". Understanding the psychobiology of NDSDs is helpful for legal councils and the litigants themselves.

\section{P40}

\section{WESTERN CANADIAN NORMS FOR COMMONLY USED} PAIN SELF-REPORT INSTRUMENTS

\section{Matthew Bailly; Gregg Tkachuk}

Department of Clinical Health Psychology, Faculty of Medicine,

University of Manitoba, Winnipeg, Manitoba

AIM: To develop and present Western Canadian norms in the form of percentiles and standardized scores for the McGill Pain Questionnaire (MPQ), Pain Disability Index (PDI), Beck Depression Inventory - II (BDI-II), Coping Strategies Questionnaire (CSQ), Chronic Pain Acceptance Questionnaire (CPAQ), Pain Catastrophizing Scale (PCS), and Tampa Scale for Kinesiophobia - 13 (TSK-13).

METHODS: Three hundred and fifty-eight patients (mean age $=47.03$, $\mathrm{SD}=12.25$ and $66 \%$ female) attending a Western Canadian multidisciplinary pain clinic were asked to complete a battery of self-report questionnaires that are routinely administered prior to receiving psychological treatment. Data were screened for violations of the assumptions of the normal distribution and outliers beyond a $\mathrm{Z}$ score of + or -3.29 were removed from further analysis on a scale by scale basis until data appeared normally distributed based on visual inspection of normality plots.

RESULTS: Tables will display percentiles and standardized residual ( $Z$ scores) corresponding to raw score means and standard error terms for each of the scale scores. For interpreting $\mathrm{Z}$ scores, scores that lie in the interval between -1 and +1 are considered normal scores. Scores above 1 are considered moderately elevated scores, whereas scores above 2 are indicative of high scores. Scores below -1 are moderately reduced scores, whereas scores below -2 are low scores. For normally distributed scores, the $z$-scores of -2 , $-1,0,1,2$ correspond to the $2 \mathrm{nd}, 16 \mathrm{th}, 50 \mathrm{th}, 84 \mathrm{th}$, and 98 th percentiles, respectively.

CONCLUSIONS: These norms have proven valuable in assisting clinicians and researchers in the processes of treatment planning and in the evaluation of treatment outcomes. It is hoped that others will benefit from access to these norms and that this study will provide a template for others to develop local norms for use within their clinics.

\section{P41}

\section{MOVED TO P3A}

\section{P42}

\section{ANTIDEPRESSANTS IN THE TREATMENT OF CHRONIC LOW BACK PAIN: QUESTIONING THE VALIDITY OF META-ANALYSES}

Owen D Williamson ${ }^{1,2}$; Doron Sagman ${ }^{3}$; Robert Bruins ${ }^{3}$; Luc Boulay ${ }^{3}$; Alexander Schacht ${ }^{4}$

1JPOCSC Pain Management Clinic, Fraser Health Authority, Surrey, Canada; ${ }^{2}$ Department of Epidemiology and Preventive Medicine, School of Public Health and Preventive Medicine, Monash University, Melbourne, Australia; ${ }^{3}$ Research and Development, Eli Lilly and Company, Toronto, Ontario; ${ }^{4}$ Global Statistical Sciences, Lilly Deutschland GmbH, Bad Homburg, Germany

AIM: To contrast the analgesic effect of duloxetine with that of antidepressants reported in other published randomized clinical trials (RCTs) and reviews in patients with chronic low back pain (CLBP).

METHODS: Results from 13 RCTs and 5 systematic reviews examining the analgesic effect of various antidepressants in CLBP were contrasted with those from 3 placebo-controlled RCTs that assessed the analgesic effect of duloxetine. Treatment effects based on the Brief Pain Inventory (BPI) average score in the duloxetine RCTs were assessed in all completers (by study and overall) and in last-observation-carried-forward (LOCF) analyses (extracted from study reports). 30\%- and 50\%-reduction response rates were compared between duloxetine and placebo.

RESULTS: Eleven different antidepressants were examined in 13 individual RCTs. Sample sizes, treatment durations, and analysis methods varied across studies. Reviews each included 5 to 9 of the RCTs and came to different conclusions regarding the analgesic effect of antidepressants: 2 found no evidence while 3 reported some evidence. The completer analysis showed greater improvements in BPI average scores with duloxetine versus placebo (significant in 2 studies). Overall, least square mean (standard error) difference between treatments was $-0.7(0.15)(\mathrm{p}<0.0001)$. Overall response rates were significantly larger with duloxetine than with placebo.

CONCLUSIONS: Due to the diversity of previous studies and the pooling methods used, the conclusions regarding the analgesic effects of antidepressants in CLBP drawn from systematic reviews must be interpreted with caution. Appropriately designed and powered studies similar to recently published duloxetine studies are recommended to demonstrate the analgesic effect of certain antidepressants.

\section{P43}

\section{CHANGES IN BISPECTRAL INDEX DURING PAIN PROCEDURE COMPARE TO REST IN PATIENTS UNABLE TO COMMUNICATE IN ICU}

Patricia Bourgault $^{1}$; Yannick Tousignant-Laflamme ${ }^{1}$;

Samuel Belzile-Lachapelle ; Émilie Gosselin ${ }^{1}$; Robin Coleman ${ }^{2}$; Flore Béland $^{2}$; François Lamontagne ${ }^{2}$; Céline Gélinas ${ }^{3}$

${ }^{1}$ Université de Sherbrooke; ${ }^{2}$ Centre hospitalier universitaire de Sherbrooke, Sherbrooke; ${ }^{3}$ Université McGill, Montreal, Quebec AIM: Pain assessment is a challenge for health care professionals, especially when patients are unable to communicate their pain. Neurophysiological measures could alleviate this, such as bispectral (BIS) index. It was previously demonstrated by our research team that nociceptive clinical procedures in the intensive care unit (ICU) and experimental nociceptive stimuli in operating room can both significantly increase BIS index. To compare changes in BIS score, physiological parameters such as heart rate (HR) and blood pressure (BP), pain observation score (CPOT) and sedation level (SAS) at rest (R1), during a pain procedure (PP) and after the painful procedure (R2) in ICU patients.

METHODS: A descriptive observational study, with repeated measures intra-subjects was conducted. Fourteen adults ( 11 men, 3 women; mean age of 68.9 years \pm 6.7 years) under sedation after cardiac surgery were included. Data were recorded during R1, PP and R2 for BIS, HR, BP, CPOT score and SAS in two conditions: $\mathrm{A}=$ with opiates analgesia and sedative medication and $\mathrm{B}=$ with only sedative medication (no opiate analgesia).

RESULTS: For condition A, mean BIS increased from $57.2 \pm 11.9$ (R1) to $62.3 \pm 13.5(\mathrm{PP})$ and returned to $56.8 \pm 11.2$ (R2). For condition $\mathrm{B}$, mean BIS also increased from $63.6 \pm 10.5$ (R1) to $71.5 \pm 13.0$ (PP) and returned to $65.2 \pm 11.8$ (R2). A similar pattern was observed for maximal BIS score during both conditions. CPOT and SAS increased during painful procedure compare to rest, contrary to HR and BP which remained stable.

CONCLUSIONS: As expected, BIS increased during painful procedure for both patients under opiates analgesia and sedative medication those only under sedation. However, this increase showed great inter-subject variability. More data are necessary. The study is ongoing and further analysis with a larger sample will be presented at completion.

\section{REFERENCES:}

1. Coleman, R., Bourgault, P., Tousignant-Laflamme, Y. La relation entre l'index bispectral et la douleur expérimentale chez l'adulte sous anesthésie générale. Canadian Pain Society. Niagara Falls, Ontario. (Avril 2011)

2. Gélinas, C., Tousignant-Laflamme, Y., Tanguay, A. \& Bourgault, P*. The use of the Bispectral Index, the Critical-Care Pain Observation Tool and vital signs for the detection of pain in sedated and mechanically ventilated ICU adults: Which measure shows the most sensitive results to nociceptive procedures? A pilot study. Intensive and Critical Care Nursing 2010;27:47-52. 


\section{P44}

THE RELATIONSHIP BETWEEN AVOIDANCE, PACING, AND TREATMENT OUTCOMES IN CHRONIC PAIN PATIENTS Douglas Cane; Mary McCarthy

Pain Services, CDHA

AIM: The relationship between pacing and psychosocial outcomes varies as a function of avoidance. While high levels of pacing and low levels of avoidance are associated with favourable outcomes, high levels of both pacing and avoidance result in poorer outcomes. This study identified chronic pain patients with high pre-treatment levels of pacing and avoidance and examined the impact of changes in these variables on posttreatment outcomes.

METHODS: Three hundred and seven chronic pain patients completed measures of activity patterns, pain intensity, affect, disability, pain control, and pain acceptance before and after a group pain management program. A two-step cluster analysis of pre-treatment activity patterns identified patients with high pre-treatment levels of avoidance and pacing. A cluster analysis of post-treatment activity scores identified patients with different combinations of post-treatment avoidance and pacing. The movement of patients from pre- to post-treatment clusters was examined and changes in avoidance, pacing, and pain acceptance were evaluated and related to outcomes.

RESULTS: Patients who reduced their use of avoidance while maintaining their pre-treatment level of pacing had better outcomes than did patients who made no changes or patients who decreased both avoidance and pacing. Increased willingness to engage in activities despite pain and a decreased need to control pain mediated these changes.

CONCLUSIONS: Decreases in avoidance alone are not associated with better treatment outcomes. Instead, decreasing avoidance while continuing to pace may produce better outcomes. Successful treatment may involve modifying the avoidance-pacing relationship. Increased pain acceptance may mediate this change and be predictive of treatment outcomes.

\section{P45}

PILOT PROJECT STUDY ON THE USEFULNESS OF AN INFORMATIONAL DOCUMENT "ARE YOU THINKING ABOUT TAKING PAINKILLERS FOR YOUR PAIN?"

Angela Carol ${ }^{1}$; Elena Matusa ${ }^{2}$; Norm Buckley ${ }^{3,4}$; Lynn Cooper ${ }^{5}$; Janice Sumpton ${ }^{6}$; Erin McGinnis ${ }^{7}$; Diane Gromala ${ }^{8}$

${ }^{1}$ Assistant Clinical Professor, McMaster University, Hamilton;

${ }^{2}$ Family Medicine, University of Western Ontario, London;

${ }^{3}$ Director, Michael GDeGroote National Pain Centre; ${ }^{4}$ Professor \&

Chair, Department of Anesthesia, McMaster University, Hamilton;

${ }^{5}$ President, Canadian Pain Coalition, Kitchener; ${ }^{6}$ Pharmacist, Canadian Society of Hospital Pharmacists, London; ${ }^{7}$ Health Promoter, Substance Misuse Prevention, Niagara Region Public Health, Thorold, Ontario; ${ }^{8}$ Associate Professor and Canada Research Chair, School of Interactive Arts \& Technology, Simon Fraser University, Surrey, British Columbia

AIM: This pilot study was developed to guide the research group in implementing a document for patients that is well-designed, understandable and addresses their fears so they may make an informed decision about considering opioids as a treatment for their pain. Patients have an important role to play in ensuring opioids are used safely. Having good informational documents to review with their doctor prior to initiating treatment will increase patients' awareness about the benefits and risks associated with an opioid trial.

METHODS: This pilot project involves 20 patients who have never been on opioids. Patients are provided with the document "Are you Thinking About Taking Painkillers for Your Pain?" to review. During their next appointment they are asked to complete an evaluation form regarding the document.

RESULTS: Thus far, $100 \%$ of participants found the document easy to read and understandable, $83 \%$ thought the document helped them decide if painkillers are a treatment to consider, answered their questions, and reduced fears about taking opioids. About a third thought the document was not helpful

CONCLUSIONS: Thus far, the document is easy to read, understandable, reduces patient's fears, answers their questions and helps them decide if painkillers are a treatment of choice. The researchers must consider the feedback of about one third of the participants before studying the impact of this document on patient's decision about the use of opioids as a treatment option for their pain.

\section{P46}

A DESCRIPTIVE ANALYSIS OF THE ART OF THE BODY MAP IN PATIENTS WITH FIBROMYALGIA

Marta Ceko ${ }^{1}$; Mary-Ann Fitzcharles ${ }^{1}$; Peter A Ste-Marie ${ }^{2}$

${ }^{1}$ McGill University Health Centre; ${ }^{2}$ University of Montreal,

Montreal, Quebec

AIM: The body map is traditionally quantitatively scored to assess pain location. We have examined body map drawings in fibromyalgia (FM) patients from the artistic perspective to determine whether the qualitative aspect provides additional insight into the experience of FM.

METHODS: The body map completed by FM patients was assessed for drawing other than shading (as per instruction) and drawing characteristics were correlated with various disease related parameters including fibromyalgia impact questionnaire (FIQ), body map quantitative score $(0-50)$, pain VAS, mood by arthritis impact measurement scale (AIMS), and pain catastrophizing scale (PCS).

RESULTS: 182 FM patients completed a body map, 172 (94\%) were female, mean age $48( \pm 10)$ years, disease duration $11( \pm 9)$ years. Body map scores were as follows: quantitative score $28( \pm 11)$, shading vs. no shading $128(70 \%)$ vs. $55(30 \%)$ respectively, circles $92(50 \%)$, other drawings 75 (41\%), outside map drawing 108 (59\%), written script 15 (8\%), and high intensity drawing $69(38 \%)$. The numbers of different drawing techniques per patient were: 1 only $-30 \%, 2-24 \%, 3-35 \%$ and $>3$ techniques $11 \%$. Descriptive assessment of the body map did not associate with any disease or psychosocial parameters.

CONCLUSIONS: Most FM patients used additional drawing techniques beyond shading in completion of the body map, although we demonstrate no association with any standard disease or psychological measures. The "artistic component" of the body map likely reflects factors other than those related to disease variables.

\section{P47}

PATTERNS OF ACTIVITY AS PREDICTORS OF TREATMENT OUTCOMES AND TREATMENT EFFECTS FOLLOWING A GROUP PAIN SELF-MANAGEMENT PROGRAM

Alexander J Clark ${ }^{1,2}$; Douglas Cane ${ }^{1}$; Darlene Davis ${ }^{1}$

${ }^{1}$ Pain Services, Capital Health; ${ }^{2}$ Department of Anesthesia, Pain

Management and Perioperative Medicine, Dalhousie University, Halifax, Nova Scotia

AIM: Considerable variability is typically observed among outcomes for pain patients following treatment. Identifying subgroups of patients differing with respect to outcomes may enhance our understanding of factors that affect patients' treatment responses. This study examined whether pain-related activity patterns (avoidance, overdoing, pacing) predicted treatment outcomes and effect sizes following a group pain management program.

METHODS: 307 chronic pain patients completed measures of activity patterns, pain intensity, affect, perceived disability, pain control, and acceptance of pain before and after a group pain management program. A two-step cluster analysis of activity patterns was used to identify clusters of patients who differed with respect to activity patterns. Outcomes and treatment effect sizes were compared for these clusters.

RESULTS: Four clusters of patients (avoidant-pacing, pacing, mixed, and overdoing) were identified. Patients identified as pacers (high scores on pacing and low scores on avoidance and overdoing) consistently obtained better outcomes than did patients in the other clusters. Specifically, patients in the pacing cluster reported lower pain levels, anxiety and disability, and greater pain control and acceptance of pain. The pacing cluster also demonstrated a larger average treatment effect size relative to the other clusters.

CONCLUSIONS: Overall, significant but small, treatment effects were observed following a group pain management program. Identifying subgroups of patients using patterns of activity resulted in better differentiation of outcomes with patients in a pacing subgroup demonstrating better outcomes and larger treatment effects. These results suggest that 
treatments that enhance pacing may produce better outcomes and larger treatment effects.

\section{P48}

EMOTIONAL PAIN AND CATASTROPHIZING INFLUENCE QUALITY OF LIFE IN FIBROMYALGIA

Neda Faregh ${ }^{1}$; Mary-Ann Fitzcharles ${ }^{1}$; Peter A Ste-Marie ${ }^{2}$ ${ }^{1}$ McGill University Health Centre; ${ }^{2}$ University of Montreal, Montreal, Quebec

AIM: Emotional pain, a critical component of the pain experience, is seldom addressed in clinical practice. We have examined the associations of emotional pain in fibromyalgia (FM) patients with quality of life, psychological status and function.

METHODS: Emotional pain, measured by the affective component of the McGill Pain Questionnaire (MPQ), was examined for association with other health, functional, and quality of life parameters in a prospective cohort of FM patients followed in a multidisciplinary pain clinic.

RESULTS: For 229 FM patients (91\% females), the MPQ (total and subsections, affective, sensory, evaluative, miscellaneous) was significantly correlated with pain visual analog scale (VAS), patient global assessment (PGA), Fibromyalgia Impact Questionnaire (FIQ), Health Assessment Questionnaire (HAQ), Pain Disability Index (PDI), Pain Catastrophizing Scale (PCS), and Arthritis Impact Measurement Scale (AIMS) anxiety and depression, but with no demographic variable other than unemployment. Stepwise hierarchical multiple regression retained FIQ, PCS, and HAQ. A MANOVA and follow-up ANOVAs indicate that affective scores contribute significantly to FIQ and PGA; evaluative scores to PGA, and the sensory scores only to HAQ. Catastrophizing contributes significantly to PGA and FIQ and provides the largest and significant Beta weights for each of the MPQ variables.

CONCLUSIONS: Higher scores on emotional pain and catastrophizing were predictors of poor quality of life, whereas sensory scores better predicted function. Emotional pain, especially when associated with high catastrophization, has negative effects on well-being in FM. Psychological interventions targeting these aspects may offer additional benefits to standard pharmacological management of pain.

\section{P49}

\section{CONTENTIOUS ISSUES IN THE DEVELOPMENT OF THE 2012 CANADIAN FIBROMYALGIA GUIDELINES}

Mary-Ann Fitzcharles ${ }^{1}$; Peter A Ste-Marie ${ }^{2}$; Don L Goldenberg ${ }^{3}$; John X Pereira ${ }^{4}$; Susan Abbey ${ }^{5}$; Manon Choinière ${ }^{2}$; Gordon Ko $^{5}$; Dwight E Moulin'; Pantelis Panopalis ${ }^{1}$; Yoram Shir ${ }^{1}$;

Johanne Proulx ${ }^{7}$

${ }^{1}$ McGill University Health Centre; ${ }^{2}$ University of Montreal, Montreal, Quebec; ${ }^{3}$ Tufts University School of Medicine, Boston, Massachusetts; ${ }^{4}$ University of Calgary, Calgary, Alberta; ${ }^{5}$ University of Toronto, Toronto; ${ }^{6}$ University of Western Ontario, London, Ontario; ${ }^{7}$ Patient Representative

AIM: Fibromyalgia (FM), a previous Cinderella diagnosis, has evoked controversy until neurophysiologic study attested to the presence of pathophysiologic abnormalities. Some embedded concepts drove debate in formulating the 2012 Canadian Fibromyalgia Guidelines. Points of contention are reviewed and resolution is discussed.

METHODS: Recommendations not achieving initial $80 \%$ approval by the advisor panel and grouped into categories are examined.

RESULTS: 1. Diagnostic criteria: Criteria for FM diagnosis, originally intended for study purposes and erroneous when applied to an individual patient, provide physician security. Consensus was achieved by recommending a clinical diagnosis can be validated with the 2010 ACR criteria. 2. Treatments: Although complementary treatment use is prevalent, limited evidence for efficacy should not preclude use. Contrary to perception, all classes of drug treatments show only modest effect, and with all classes of antidepressants demonstrating some efficacy. Specific drug choices should be patient-tailored according to physicians' knowledge and evidence for efficacy. 3. Terminology: The terminology of antidepressants and anticonvulsants is engrained in clinical practice without recommendation to identify them as pain modulators. 4. Past/triggering events: Previous negative lifetime events, even those perceived as triggers, should be acknowledged but without excessive focus detracting from clinical care.
5. Work and disability: Continued work is ideal and encouraged, with recommendation for a rehabilitation program for those not working to improve function and possible return to work was recommended.

CONCLUSIONS: The 2012 Canadian FM Guidelines will help dispel false notions that may hinder management, will facilitate rational patient care, and galvanize the healthcare community.

\section{P50}

A PARADIGM CHANGE FOR THE HEALTHCARE, PATIENT, AND THIRD PARTY PAYER COMMUNITY REFLECTED BY THE 2012 CANADIAN FIBROMYALGIA GUIDELINES

Mary-Ann Fitzcharles ${ }^{1}$; Peter A Ste-Marie ${ }^{2}$; Don L Goldenberg ${ }^{3}$; John X Pereira ${ }^{4}$; Susan Abbey; Manon Choinière ${ }^{2}$; Gordon Ko ${ }^{5}$; Dwight E Moulin'; Pantelis Panopalis ${ }^{1}$; Johanne Proulx ${ }^{7}$; Yoram Shir ${ }^{1}$

${ }^{1}$ McGill University Health Centre; ${ }^{2}$ Université de Montréal, Montreal, Quebec; ${ }^{3}$ Tufts University School of Medicine, Boston, Massachusetts; ${ }^{4}$ University of Calgary, Calgary, Alberta; ${ }^{5}$ University of Toronto, Toronto; ${ }^{6}$ University of Western Ontario, London, Ontario; ${ }^{7}$ Patient representative

AIM: The 2012 Canadian Fibromyalgia Guidelines represent a considerable paradigm change for healthcare professionals, patients, and third party payers. New concepts presented by these guidelines will be discussed.

METHODS: Highlights reflecting change in the clinical approach to fibromyalgia (FM) are presented.

RESULTS: 1. FM is a clinical construct, which should be positively diagnosed in the primary care setting without requirement for specialist confirmation or follow up. Only the exceptional patient should be referred for specialist opinion. The tender point count, a subjective assessment without scientific basis, should no longer be used to establish a diagnosis, and may be eliminated from the clinical evaluation. 2. Ideal patient care should be centered in the primary care setting, with encouragement to reduce excessive healthcare contact and investigations. 3 . The value of non pharmacologic treatments is emphasised with focus towards self management strategies and exercise activity, and with a shift away from primarily pharmacological treatments, often associated with side effects similar to symptoms of FM. Drug combinations with cautious low dosing should be considered. 4. The culture of disablement surrounding FM is now questioned with call for a societal change to focus towards patient motivation and retention in normal life pattern, with the acknowledgement that symptoms will likely persist and fluctuate.

CONCLUSIONS: Patients and the healthcare community should approach the diagnosis and clinical care of FM patients with a positive attitude incorporating good healthcare concepts in order to reduce the negative effects of "sickness labeling".

REFERENCES:

1. 2012 Canadian Fibromyalgia Guidelines may be consulted at: http:// www.canadianpainsociety.ca/pdf/Fibromyalgia_Guidelines_2012.pdf

\section{P51}

CHALLENGING CONVERSATIONS BETWEEN PHYSICIAN AND PATIENT AROUND OPIOID PRESCRIBING FOR CHRONIC NON-CANCER PAIN

John F Flannery ${ }^{1}$; Dawn Martin²; Andrea D Furlan ${ }^{1,3}$

${ }^{1}$ Toronto Rehabilitation Institute - UHN, Division of Physiatry, University of Toronto; ${ }^{2}$ University of Toronto; ${ }^{3}$ Institute for Work \& Health, Toronto, Ontario

AIM: The aim of this study was to identify what physicians perceive to be challenging conversations with patients when prescribing opioid therapy for chronic non-cancer pain and to develop educational materials to help increase physician comfort and confidence in these areas. Challenging conversations were defined as "sticky points" where the physician felt uncomfortable, reluctant or awkward in holding a conversation resulting in the physician either avoiding the conversation or inappropriately managing the conversation.

METHODS: The base for the identification of challenging conversations was the Canadian Opioid Guideline and the Opioid Manager. The Opioid Manager is a point of care tool for providers prescribing opioids for chronic non-cancer pain and it condenses the key elements of the Canadian Opioid Guideline. The methods to identify challenging conversations 
included: electronic survey of physiatrists in Ontario, feedback from experts in the area of chronic pain management and consultation with a communication and curriculum specialist.

RESULTS: The survey and the feedback from the experts revealed six "sticking points" when initiating an opioid trial that provided challenges for physicians:

1-Goal setting for chronic opioid therapy

2-Introducing the opioid risk tool

3-Asking for urine to conduct a urine drug screening test

4-Discussing unexpected results of urine drug screening

5-Setting limits on opioid titration

6-Stopping or tapering opioid therapy.

Videos were created to depict the six sticking points most commonly seen when prescribing opioids. Each office visit demonstrates a different challenging conversation between doctor and patient.

CONCLUSIONS: The six videos will be used as a tool for educating physicians to improve their communication skills around opioid prescribing for chronic non-cancer pain.

\section{P52}

OFF LABEL USE OF PREGABALIN (LYRICA®) IN PATIENTS WITH CHRONIC NON-CANCER PAIN: AN EXPLORATORY STUDY

Hili Giladi $^{1}$; Manon Choinière²; Mary-Ann Fitzcharles ${ }^{1}$;

Mark Ware ${ }^{1}$; Yoram Shir ${ }^{1}$

${ }^{1}$ The Alan Edwards Pain Management Unit, McGill University

Health Centre, Montreal; ${ }^{2}$ Centre de recherche du Centre

hospitalier de l'Université de Montréal (CRCHUM), Hôtel-Dieu,

Montréal, Quebec

AIM: No previous study has systematically examined off-label prescriptions of pregabalin in patients with chronic non-cancer pain. Our aims were: a) to assess the proportion of chronic pain patients taking pregabalin for pain conditions not included in Health Canada's recommendations for pregabalin use (off- label); b) to determine pain conditions that prompted the prescription of pregabalin; c) to explore patients' perspectives of the benefits and impediments of pregabalin in on- and off-label users.

METHODS: Subjects were recruited from two registries of chronic pain patients treated in tertiary care clinics i.e., the Quebec Pain Registry and the AEPMU Fibromyalgia Registry. Present and past year pregabalin users were identified and contacted. Data were collected from the registries and from questionnaires filled by consenting individuals. The questionnaires included items relating to the use of pregabalin and its perceived benefits.

RESULTS: 357 out of 4339 screened patients (8.23\%) were found eligible for the study. Three quarters of them (265/357) used pregabalin for a pain problem not indicated by Health Canada (off- label). The most prevalent condition for pregabalin use was non-neuropathic lumbar back pain $(103 / 357 ; 28.85 \%)$. There were no significant differences between the groups of on- and off-label users in their perceived satisfaction from pregabalin therapy and its effect on function and quality of life.

CONCLUSIONS: Despite the specific Health Canada indications for pregabalin prescription, it is mainly used for off-label indications, notably for mechanical low back pain. Patients taking pregabalin on and off-label reported that they derived similar benefits from it.

\section{P53}

INFORMING THE DESIGN OF A CHRONIC PAIN WEBBASED SELF-MANAGEMENT PORTAL

Amédé Gogovor ${ }^{1}$; Regina Visca ${ }^{2}$; Iphigenia Symeonidis ${ }^{1}$; Sara Ahmed ${ }^{1}$

${ }^{1}$ McGill University; ${ }^{2}$ Centre of Expertise in Chronic Pain, RUIS McGill, Montreal, Quebec

AIM: Several barriers to optimal management of chronic pain (CP) have been documented in the literature and include limited access to health care providers. Health information technology offers an opportunity to provide self-management support and regular monitoring and exchange of information between the patient and care team. The objectives of the study were to identify gaps in CP management and features of a web-based tool that would be beneficial for providing patient self-management support and improving CP management by the care team.
METHODS: A structured literature review and focus groups with individuals with $\mathrm{CP}$ and with health professionals working in CP rehabilitation and management. The following databases were searched: Medline, Embase, PsycINFO, Cinahl, Cochrane library, Amed, ISI Web of Science. Narrative synthesis and thematic analysis were conducted and themes and concepts were linked to features.

RESULTS: Successful health portals require the integration of interactive features with personalization capabilities and some level of monitoring by health professionals. In terms of content, individuals with CP suggested a common basic section and different sections specific to types of $\mathrm{CP}$ while health professionals suggested a stratified access to information based on patients' stage of advancement. Unlike health professionals, individuals with $\mathrm{CP}$ underscored the importance of nonmedical information (e.g. ergonomics, stress management, diet for $\mathrm{CP}$ patients, legal procedures).

CONCLUSIONS: Interactivity and personalization appear to be important portal features. However, currently existing systems are limited in the integration of personal health records. The results derived from this study will be used to develop and implement a web-based CP self-management portal.

\section{P54}

ORGANIZATIONAL IMPACT OF THE CANADIAN GUIDELINE FOR SAFE AND EFFECTIVE USE OF OPIOIDS FOR CHRONIC NON-CANCER PAIN: SURVEY ON OPIOID POLICY

lan Goldstine ${ }^{1}$; Cheryl Arratoon ${ }^{2}$; Norm Buckley ${ }^{3}$;

Amol Deshpande ${ }^{4}$; Paula Robeson ${ }^{2}$

${ }^{1}$ Workers Compensation Board of Manitoba, Winnipeg, Manitoba;

${ }^{2}$ Canadian Centre on Substance Abuse, Ottawa, Ontario; ${ }^{3}$ Director, Michael G DeGroote National Pain Centre; Professor \& Chair, Department of Anesthesia, McMaster University, Hamilton, Ontario; ${ }^{4}$ Comprehensive Pain Program, University Health Network \& University of Toronto, Toronto, Ontario

AIM: The Canadian Opioid Guideline was published in 2010 to address public health and clinical care issues with the use of opioids. As part of the knowledge transfer process, six working groups were established to disseminate and evaluate the guideline. One working group is focused on the implications of the guideline for policy-makers and third-party payers. The working group developed a survey for Canadian governmental and nongovernmental agencies to determine awareness of the guideline and the extent to which policies in these agencies support implementation of the guideline recommendations.

METHODS: We obtained contact information for the Deputy Ministers of Health and the Medical Directors of the Workers Compensation Boards in our ten provinces and three territories. The on-line survey was developed and distributed using FluidSurvey (Ottawa, Canada). Ministers and Medical Directors were notified by email of the URL for the survey site and asked to complete the survey. The survey site was accessible for 27 days and could be completed anonymously.

RESULTS: Eight survey responses (30\%) plus free-text comments were received. Most respondents had opioid policies in place and were aware of the guideline. Specific barriers to implementation included clinician belief that the guideline was not relevant to specific patients and slow dissemination of recommendations into clinical practice.

CONCLUSIONS: While many organizations have existing policies to address the use of opioids, barriers still exist to implementing evidencebased guideline recommendations.

\section{P55}

SUBCUTANEOUS LIDOCAINE INFUSION FOR CHRONIC PAIN: THE LIDO-CHRON STUDY

Kyle Grieve $^{1}$; Donna Kay Buna ${ }^{2}$

${ }^{1}$ East Kootenay District Hospital, Interior Health Authority; ${ }^{2}$ Vancouver Island Health Authority, Regional Pain Program, Victoria site, British Columbia

AIM: The Vancouver Island Health Authority (VIHA) Regional Pain Program offers intermittent subcutaneous lidocaine infusions to ambulatory patients with chronic pain unresponsive to usual therapies. A literature review revealed that no studies have examined lidocaine given by the subcutaneous route in chronic pain patients. Our objective was to estimate 
the extent and duration of pain relief in patients receiving intermittent lidocaine infusions in our program.

METHODS: This single centre observational study enrolled patients receiving intermittent subcutanous lidocaine infusions at an ambulatory pain clinic. Patients were surveyed each day from one infusion until the next (21 days). The primary outcome was change in pain intensity using a numerical rating scale from 0 to 10 .

RESULTS: Thirty-one patients were included in the analysis. A mean pain reduction of 1.5 points was observed ( $95 \% \mathrm{CI} 0.8$ to 2.2 ; $\mathrm{p}<0.001$ ) the day after lidocaine infusions with the greatest mean reduction seen on day $8(2.1,95 \%$ CI 1.2 to $2.9 ; \mathrm{p}<0.001)$. Breakthrough pain medication use was also reduced and function improved. By day 13 the reductions in all measures were no longer statistically significant. Adverse effects such as fatigue, taste changes, numbness and dizziness were common immediately post infusion ( $66 \%$ incidence), but resolved by day 4 post-infusion.

CONCLUSIONS: Intermittent subcutaneous lidocaine may reduce the severity of neuropathic pain in patients unresponsive to usual therapies. A clinically meaningful benefit in pain reduction may last for 7 to 13 days. Adverse effects were common, but resolved day 4 post infusion.

\section{P56}

\section{MY ARTHRITIS HURTS BUT I ACCEPT IT: DOES PAIN ACCEPTANCE PREDICT ENDURANCE PHYSICAL ACTIVITY AMONG WOMEN WITH ARTHRITIS?}

Nancy C Gyurcsik ${ }^{1}$; Danielle R Brittain ${ }^{2}$

${ }^{1}$ University of Saskatchewan, College of Kinesiology, Saskatoon, Saskatchewan; ${ }^{2}$ University of Northern Colorado, School of Human Sciences, Community Health Program, Greeley, Colorado, USA

AIM: Most women with arthritis do not adhere to the recommended dose of physical activity (PA) for disease self-management (150+ minutes/ week). Arthritis pain is a frequently reported barrier to PA, yet the relationship between pain and adherence is inconsistent. Pain acceptance, an aspect of psychological flexibility involving a willingness to pursue activities despite pain, may facilitate adherence. The purpose was to investigate whether pain acceptance predicted PA, after controlling for arthritis-related pain intensity.

METHODS: Participants were 117 women (Mage $=48.72 \pm 14.44$ years) with medically-diagnosed arthritis who completed online measures of demographics, pain intensity, and pain acceptance at Time 1, followed by endurance PA two weeks later.

RESULTS: Participants reported a moderate level of pain intensity $(M=5.42 \pm 1.78)$, were accepting of their pain $(M=68.52 \pm 17.55)$, and engaged in 175 minutes of $\mathrm{PA} /$ week. To investigate the study purpose, a hierarchical multiple regression analysis to predict PA was conducted in which pain intensity was entered first, followed by pain acceptance in step two. The overall model was significant, $F(2,114)=6.82$, $p<0.01$, with pain acceptance being the sole significant predictor $(\beta$ stand. $=0.35, \mathrm{p}<0.01$ ).

CONCLUSIONS: Findings were the first to illustrate a predictive relationship between arthritis pain acceptance and PA. Acceptance may be an important facilitative process that helps women adhere to PA, regardless of their pain. Future research should continue to examine pain acceptance as well as other disease-related psychological processes that help individuals pursue PA adherence (e.g., illness perceptions; other aspects of psychological flexibility) in order to better self-manage their arthritis.

Funded by SSHRC grant \#410-2005-2292.

\section{P57}

DEVELOPMENT OF AN ONLINE CHRONIC PAIN PROGRAM FOR INDIVIDUALS WITH A MILITARY/RCMP BACKGROUND

Pamela L Holens ${ }^{1}$; Heather Simister ${ }^{1}$; Kristen Klassen ${ }^{1}$;

Amber Gilberto $^{2}$

${ }^{1}$ University of Manitoba; ${ }^{2}$ Deer Lodge Centre OSI Clinic, Winnipeg, Manitoba

AIM: The objectives of this pilot study were: 1) to develop an online chronic pain management program tailored to a military/RCMP population, and 2) to assess its acceptability and appropriateness for this population.

METHODS: Modifications were made to a pre-existing AcceptanceBased Behavioral Therapy (ABBT) for chronic pain to tailor it to a
military/RCMP population. Ten participants were recruited to complete the program and offer their insights and suggestions regarding its acceptability, appropriateness, and efficacy. Participants included veterans and actively serving members of both genders with a median age of 43.5 years. RESULTS: Nearly fifty percent of individuals approached regarding participation in the study declined to participate or dropped out early in the program, with the most frequent reasons being that the online approach was not a good fit for them or they were currently focusing on other concerns. Those who completed the online modules reported finding them informative and helpful. Participants related well to the military/RCMP vignettes in the program, but the ideas of acceptance and mindfulness were noted to be dissonant with their training and experiences.

CONCLUSIONS: Our online therapy for military/RCMP with chronic pain was deemed appropriate for some, but by no means all, members of this population. Some members preferred a face-to-face option, while others reported co-morbid mental health concerns were their current priority. For those comfortable with the online approach, the material in this program was generally deemed acceptable and appropriate, with the possible exception of the concepts of acceptance and mindfulness.

\section{P58}

PAIN PREVALENCE AMONG IN-HOSPITAL PATIENTS

Kimberly Jabusch; Barbara Lewthwaite; Lynda Mandzuk; Karen Schnell-Hoehn; Barbara Wheeler

St Boniface Hospital, Winnipeg, Manitoba

AIM: To quantify the prevalence of pain among adult patients on a single day in an urban tertiary care hospital in central Canada.

METHODS: This descriptive, cross-sectional study used a convenience sample $(\mathrm{N}=88)$ of medical-surgical patients who completed six demographic questions and the Short Form-Brief Pain Inventory (SF-BPI).

RESULTS: Pain prevalence was $70.4 \%$. The mean pain severity score was 3.76 (SD 2.88) and mean pain interference score on daily activities was 4.56 (SD 3.93). The most frequently identified site of pain was the lower extremities $(n=15,28 \%)$. Women had higher mean scores on pain "right now" compared to men $(\mathrm{p}<0.05)$. The sample majority $(\mathrm{n}=81)$ indicated hospital staff asked about the presence of pain. Seventy-nine percent $(n=57)$ reported hospital staff "always" did everything they could to help manage pain. Eighty-five percent $(\mathrm{n}=72)$ selected "always" or "usually" to describe their ability to be involved in deciding pain treatments. The mean pain relief score from treatments was $61 \%$ (SD 34.79). Significant positive correlations were found between pain intensity ratings and pain interference on all daily activities $(p<0.001)$. Duration of pain was significantly correlated to higher "least" pain scores and greater pain interference with general activity $(\mathrm{p}<0.05)$.

CONCLUSIONS: Pain prevalence remains high with significant interference on activities of daily living. The study provides baseline data to direct future initiatives at improving pain management.

\section{P59}

CHANGE OF KNOWLEDGE, ATTITUDES AND BEHAVIOURS AMONG ONTARIO PHYSIATRISTS IN MANAGING CHRONIC NON-CANCER PAIN WITH OPIOIDS BEFORE AND AFTER THE RELEASE OF THE CANADIAN OPIOID GUIDELINE

Alice Kam ${ }^{1}$; John F Flannery ${ }^{1,2}$; Mike Allen ${ }^{3}$; Andrea D Furlan ${ }^{1,2,4}$

${ }^{1}$ Division of Physiatry, University of Toronto; ${ }^{2}$ Toronto

Rehabilitation Institute - UHN, Toronto, Ontario; ${ }^{3}$ Dalhousie University, Halifax, Nova Scotia; ${ }^{4}$ Institute for Work \& Health, Toronto, Ontario

AIM: To identify how the Canadian Opioid Guideline's dissemination and implementation strategies affected knowledge, attitudes and behaviours of Ontario Physiatrists regarding the use of opioids for Chronic Non-Cancer Pain.

METHODS: We applied the same electronic survey to Physiatrists practicing in Ontario, before and 2 years after the release of the Guideline.(1) The guideline was disseminated by the College of Physicians and Surgeons of Ontario and it was delivered through a website, scientific journal publications, presentation at scientific meetings, continuing medical education, and various community workshops.

RESULTS: The response rate was low: 25\% (35/142) before and 19\% 
(28/150) after the Guideline. The demographics of the first and second survey responders were similar to the Ontario physiatrist demographics. Two years after the release of the Guideline, $72 \%$ (18/25 subjects) have heard of the Guideline and 28\% (7/25) physicians believed that the guideline changed their practices. However, there were no significant changes regarding responders' level of confidence in prescribing opioids, their definitions of chronic non-cancer pain, their monitoring parameters, frequency, decision and selection of opioids prescription.

CONCLUSIONS: The transfer of research evidence into practice is a complex process. The low response rate and small absolute numbers of responses limits definitive conclusions. However it appears that despite effective dissemination, current guideline implementation strategies do not lead to knowledge, attitudes and behaviours changes of practicing physicians. Identifying strategies to optimize implementation capacity is needed.

REFERENCES:

1. http://nationalpaincentre.mcmaster.ca/opioid/

\section{P60}

PSYCHOMETRIC DEVELOPMENT OF THE CHRONIC PAIN ACCEPTANCE SELF-RATING SCALE (CPASRS): RESULTS OF THE FOCUS GROUP

\section{Audrey Kinzel; Laurie Hellsten; Randy Duncan}

University of Saskatchewan, Saskatoon, Saskatchewan

AIM: Acceptance of chronic pain is embraced by some individuals allowing them to live meaningfully and purposefully with pain. The purpose of this multiphase study is to accumulate psychometric evidence for the CPASRS and develop treatment interventions to facilitate management of chronic pain. The CPASRS will provide professionals/clinicians with a valid and useful measure to identify clients' acceptance related attitudes, beliefs, emotions, and behaviours. This information will allow clinicians to tailor interventions thus increasing treatment efficiency whereby client distress will reduce while increasing quality of life. Phase one involved item content reviews. Results of the current phase, the focus group, are presented.

METHODS: The purpose of the focus group was to have individuals knowledgeable in chronic pain, dialogue about the CPASRS. The transcript of the session served as data. Focus group participants addressed issues including face validity (instruction clarity, scale length), completion time, and item wording.

RESULTS: Participants were recruited via experts from previous rounds. Participants dialogued primarily on clarity of items and instructions. The transcript was analyzed using the methods recommended by Braun and Clarke (2006). Consensus was achieved on suggested revisions though overall the wording was clear and items captured the operational subscale definitions.

CONCLUSIONS: Results of the focus group lead to minor revisions in the CPASRS. Next steps are a pilot study and development of interventions to facilitate chronic pain management. Knowledge about an individuals' level of acceptance and related constructs is predicted to facilitate appropriate interventions to assist the individual living with pain. The CPASRS will provide this information.

\section{P61}

THE EFFECT OF AN INFORMATION AND RELAXATION ON PRE-PROCEDURAL ANXIETY AND PAIN DURING A NERVE BLOCK FOR PATIENTS WITH CHRONIC PAIN:

\section{A RANDOMIZED CONTROLLED TRIAL.}

Louise Lamb; Neda Fareg; Roderick J Finlayson

\section{McGill University Health Centre, Montreal, Quebec}

AIM: Although nerve blocks can be beneficial in the treatment of chronic spinal pain, patients can often experience significant peri-procedural anxiety, which can result in greater discomfort during the block and procedural difficulty for the physician. We therefor sought to examine the effects of a combined relaxation and information intervention on a population of chronic pain patients undergoing blocks for spinal pain.

METHODS: Patients were randomized to either usual care or a treatment group which received a preparatory information session and a relaxation training intervention four days before the nerve block procedure.
Measurements included Trait anxiety, State anxiety and catastrophization on enrolment and pre-block, pain score during the procedure and difficulty in performing the procedure as rated by the physician who was blinded to allocation.

RESULTS: Of 61 patients recruited, 26 patients in the treatment group and 30 in the control group completed the study. Both groups were found to have elevated scores on measures of anxiety (41.96 SD 11) and catastrophization (26.49 SD 11.23), and significant correlations were found between State anxiety, catastrophization, and procedural pain (all p<0.05). Contrary to what had been reported in other populations undergoing painful procedures, we found no effect of our intervention on any of the measures, and no intergroup differences were found.

CONCLUSIONS: We theorize that our population of patients suffering from chronic spinal pain, because of their elevated levels of anxiety and catastrophization, would require a more robust intervention to reduce the distress associated with nerve blocks.

\section{P62}

\section{HEART RATE AND HEART RATE VARIABILITY CHANGES DURING LIDOCAINE INFUSION IN CHILDREN WITH COMPLEX REGIONAL PAIN SYNDROME}

Gillian Lauder $^{1}$; Chris Brouse ${ }^{2}$; Matthias Görges ${ }^{2}$; Michele Smith ${ }^{1}$; Sabina Walker ${ }^{3}$; Nicholas West ${ }^{4}$

${ }^{1}$ Department of Anesthesia, BC Childrens Hospital (BCCH);

${ }^{2}$ Department of Electrical and Computer Engineering, University of British Columbia; ${ }^{3}$ Institution pending; ${ }^{4}$ Department of Anesthesiology, Pharmacology and Therapeutics, University of British Columbia, Vancouver, British Columbia

AIM: Complex Regional Pain Syndrome (CRPS) is an immuno-neurological condition in which pathophysiological changes produce severe pain and allodynia. CRPS treatment requires interdisciplinary care focused on paced physiotherapy. Physiotherapy is difficult to initiate if allodynia is so severe that the affected limb cannot be touched. At BCCH, children with allodynia secondary to CRPS receive 3 lidocaine infusions, with a low $(5 \mathrm{mg} / \mathrm{kg})$, then medium $(7 \mathrm{mg} / \mathrm{kg})$, and then high $(9 \mathrm{mg} / \mathrm{kg})$ dose. These have proven effective at reducing allodynia. A recent study has demonstrated increased heart rate (HR) and decreased heart rate variability (HRV) in adult CRPS patients [1]. We aim to examine HR/HRV changes during lidocaine infusions in children with CRPS.

METHODS: With institutional ethical approval, HR/HRV data will be retrospectively analysed from cardiovascular parameters routinely recorded in all cases monitored in $\mathrm{BCCH}$ perioperative areas. Data will be analysed to estimate the autonomic state during lidocaine infusions for CRPS patients, using standard HRV measures applied in the time and frequency domains. HR/HRV measures will be calculated at baseline (1 to 5 minutes before infusion), at initial response (after 20 minutes) and final response (during last 10 minutes).

RESULTS: Data have been collected during 8 low, 4 medium and 3 high dose lidocaine infusions administered in 7 children, median age 14 years (range 8 to 16 years). HR/HRV data are yet to be analysed.

CONCLUSIONS: Data analysis will reveal if HR and HRV change during lidocaine infusions in children with CRPS.

REFERENCES:

1. Terkelsen, et al. Anesthesiology 2012;116:133-46

\section{P63}

\section{WITHDRAWN}

\section{P64}

\section{RETROSPECTIVE ANALYSIS OF PATIENTS WITH ABDOMINAL MALIGNANCIES WITH PAIN AT TERTIARY CARE CANCER CENTER.}

Seema Mishra; Sushma Bhatnagar; Syed Mehmood Ahmad; Saurabh Joshi IRCH, AIIMS, Ansari Nagar, New Delhi, India

AIM: Abdominal cancers are one of the leading causes of morbidity and mortality among cancer patients worldwide. As most of these patients present in the hospital at a late stage of the disease when the curable options are deemed ineffective or unavailable, the role of palliative care in general and 
pain management in particular has to be addressed to alleviate the sufferings of the patients in the last days of life. Our aim is to evaluate and analyse the patients with abdominal malignancies for pain and other distressing symptoms in pain and palliative care ward at a tertiary care cancer center. METHODS: This is a retrospective study conducted in pain palliative care unit of IRCH, AIIMS, India. Patients of all age groups with abdominal malignancies suffering from pain and other distressing symptoms were included in the study. Clinical data were abstracted from the medical records demographic profile, disease status, treatment received, reason for admission, clinical symptoms and its management, psycho-social factors, outcome, satisfaction with the hospital admission, any palliative intervention and were recorded.

RESULTS: A total of 235 patients suffering different types of abdominal malignancies were admitted in palliative care ward. Maximum number of patients were of cancer of hepatobiliary system (111), followed by colorectal carcinoma (50), carcinoma pancreas (35), carcinoma stomach (25) and others (09). Majority of the patients came with the complaints of inadequate pain relief with the present medications (35.3\%). Majority of $t$ patients were suffering from visceral type of pain $(48.1 \%)$ followed by mixed type (46\%), somatic (3.4\%) and pure neuropathic type (1.7\%). Pain score on visual analogue scale (VAS) were noted on admission and on discharge from the palliative care ward. $48.5 \%$ of the patients had VAS score varies from 3 to 7 and $17.4 \%$ of the patients had VAS $>7$ on admission. On discharge none of the patients had VAS $>3$ indicating that adequate pain relief was attained in all patients either with pharmacological interventions or other interventions done in the ward. Interventions either for pain relief or for other symptoms were done in $45.1 \%$ of the patients. $17.9 \%$ of the patients were admitted for supportive care and detailed workup. Pain management interventions were done with pharmacological opioid dose titration and timely intake oral morphine with appropriate adjuvants. $33.2 \%$ of the patients received interventions in the form of interventional techniques such as celiac plexus block, ,hypogastric plexus block, ganglion impar block, epidural steroid, intrathecal implant placement and lumbar

plexus block. $51.2 \%$ of the patients had a stay of $<3$ days, $23.4 \%$ for 3 to 5 days while $24.6 \%$ had a stay of $>5$ days in the palliative care ward. Only $23 \%$ of the patients were aware of the diagnosis and $17 \%$ aware of the prognosis of the disease at the time of the admission as the relatives did not wish to discuss the diagnosis and the prognosis. During the stay in the ward counselling of the patients and relatives were done and the majority $(>85 \%)$ of the patients were explained about the disease. Most of the patients $(>95 \%)$ were pain free, stable and happy at the time of discharge from the hospital.

CONCLUSIONS: Patients with abdominal malignancies are presented to the cancer center in advanced stages in India. Uses of multimodal approach in pain and symptom management in these patients lead great satisfaction among the patients and physicians.

\section{P65}

\section{SOME NOTES ON THE PERSISTENCE OF SUBCUTANEOUS B12 ANALGESIC EFFECTS IN CHRONIC PAIN: CASE STUDIES}

lanice Montbriand $^{1}$; M Montbriand ${ }^{2}$

${ }^{1}$ University of Northern British Columbia, Prince George, British

Columbia; ${ }^{2}$ Lakeshore Medical Clinic, Regina, Saskatchwan

AIM: Vitamin B12 injections have an analgesic effect in subsets of chronic pain. However, research has not investigated their analgesic effects over time, after the initial two week loading period. The goal of this case series is to present a follow-up on the persistence of the analgesic effects of selfadministered subcutaneous B12 in a small chronic pain sample, and to include some recommendations for further research.

METHODS: Participants had various moderate to severe chronic pain conditions and inadequately controlled pain levels. They were instructed on self-administration of subcutaneous B12 shots (1000 mcg subcutaneously daily for a 2 week period, then as often as needed). Follow-up concerned reduction in pain, persistence of pain relief after the initial loading period, changes in activity levels noted, etc.

RESULTS: Respondents found that their pain relief from the B12 injections generally lasted an average of 3 days before more injections were needed. Certain subgroups seemed to benefit most from the injections. Specific case studies are discussed.
CONCLUSIONS: The use of self-administered B12 injections in chronic pain holds promise as an adjunct treatment. Those unable to tolerate other treatments may particularly benefit; elderly patients would be well represented here. It is suggested that participants be taught to self-administer B12, 1000 mcg subcutaneously for a 2 week daily loading series, and then as needed. It is also suggested that further research may be needed to investigate whether those with comorbid mood disorders and/or pain with a neuropathic component benefit most from this treatment.

\section{P66} CANADIAN MULTI-CENTRE COHORT STUDY TO
DETERMINE THE LONGTERM OUTCOMES OF THE
MANAGEMENT OF CHRONIC NEUROPATHIC PAIN DE Moulin ${ }^{1}$; AJ Clark ${ }^{2}$; A Gordon ${ }^{3}$; M Lynch ${ }^{2}$; PK Morley-Forster ${ }^{1}$; H Nathan ${ }^{4}$; C Smyth ${ }^{4}$; C Toth $^{5}$; E VanDenKerkhof ${ }^{6}$; A Gilani ${ }^{7}$; MA Ware ${ }^{8}$

${ }^{1}$ Western University, London, Ontario; ${ }^{2}$ Dalhousie University, Halifax, Nova Scotia; ${ }^{3}$ University of Toronto, Toronto; ${ }^{4}$ University of Ottawa, Ottawa, Ontario; ${ }^{5}$ University of Calgary, Calgary, Alberta; ${ }^{6}$ Queens University, Kingston; ${ }^{7}$ McMaster University, Hamilton, Ontario; ${ }^{8}$ McGill University, Montreal, Quebec

AIM: To determine the long-term outcomes of the management of chronic neuropathic pain at tertiary pain centres.

METHODS: An observational cohort study of patients with chronic neuropathic non-cancer pain, recruited from 7 Canadian pain centres, was conducted. Patients were treated according to standard guidelines and followed at 3, 6, 12 and 24 months. Standard outcome measures for pain (Brief Pain Inventory[BPI]), disability (Pain Disability Index), mood (Profile of Mood States), quality of life (SF-12) and overall treatment satisfaction (Global Satisfaction Scale) were administered with the primary outcome measure designated as the composite of $30 \%$ reduction in average pain intensity and one point drop in the mean interference scale score of the BPI at 12 months relative to baseline.

RESULTS: Of 789 patients recruited, mean age was $53.5 \pm 14.2$ years $(55 \%$ female) with mean duration of pain $58.5 \pm 69.8$ months. Major pain syndromes were central pain $(10.1 \%)$, mononeuropathies $(45.1 \%)$, asymmetrical polyneuropathies $(25.7 \%)$ and symmetrical polyneuropathies $(19.1 \%)$. Mean average pain intensity ( 0 to 10$)$ at baseline was $6.1 \pm 1.9$. All standard outcome measures showed statistically significant improvement at 12 months relative to baseline $(\mathrm{p}<0.05)$. However, only $23.7 \%$ attained clinically significant improvement in pain and function at 12 months as the primary outcome measure.

CONCLUSIONS: Long-term observational studies may have greater external validity than short-term randomized controlled trials. However, our present treatment modalities only provide significant benefit in about a quarter of neuropathic pain patients presenting to tertiary care pain clinics. More effective analgesic agents are required to manage neuropathic pain.

ACKNOWLEDGEMENTS: This study was supported by grants from Canadian Foundation for Innovation (\#7878) and Pfizer Canada.

\section{P67}

\section{MOVED TO P29A}

\section{P68}

AN RCT OF TBAR FOR DPN: CAN THERMAL BIOFEEDBACK BE SUCCESSFUL IN DIABETIC NEUROPATHY

Angela F Pfammatter ${ }^{1,2}$; Kenneth Lofland ${ }^{3,4,5}$; Sara Dittoe Barrett ${ }^{6}$; Christine Gagnon ${ }^{5,6}$

${ }^{1}$ Capital District Health Authority, Halifax, NS; ${ }^{2}$ Rosalind Franklin University of Medicine and Science, North Chicago; ${ }^{3}$ Northshore Integrative Health Care; ${ }^{4}$ Rush University Medical Center; ${ }^{5}$ Northwestern University Feinberg School of Medicine; ${ }^{6}$ Rehabilitation Institute of Chicago, Chicago, Illinois

AIM: Current literature lacks effective, direct treatments for the painful condition of diabetic peripheral neuropathy (DPN). To date, treatment options mask the painful symptoms without improving the severity of the disease (Harati, 2007). Given that Thermal Biofeedback Assisted Relaxation (TBAR) has been successfully used in other conditions where 
dysfunctional blood perfusion is implicated, the current study aimed to explore the use of TBAR as a feasible treatment option for DPN.

METHODS: 21 participants diagnosed with DPN were randomly assigned to an experimental and control condition. The experimental group attended six sessions of TBAR. The control condition attended the same number of sessions and asked to talk about benign topics with the experimenter. All participants were asked to rate their pain at the beginning and end of each session. Hand and foot temperature were measured during each session to examine within session change. Between session changes were examined using temperatures across the sessions. It was hypothesized that the experimental group would show greater increases in temperature and greater decreases in pain than the control group.

RESULTS: A repeated-measures ANOVA demonstrated that pain ratings within session decreased greater in the experimental group, though the large effect did not reach statistical significance, likely due to an inadequate sample size (i.e., 21 participants studied, 48 needed based on power analysis). Similarly, results demonstrated a greater increase in temperature both within and between sessions, though the large effect did not reach significance.

CONCLUSIONS: Though findings are limited by a small sample size, this study shows the potential effect TBAR may have for DPN. This is the first study of its kind and further research with a larger sample size is needed to make further conclusions.

FOOTNOTES/REFERENCES: Harati, Y. (2007). Diabetic Neuropathies: Unanswered questions. Neurologic Clinics, 25.

\section{P69}

\section{PARENT JUDGMENTS OF INFANT PAIN IN THE PEDIATRIC} CLINIC

Rebecca Pillai Riddell ${ }^{1}$; David Flora ${ }^{1}$; Sara Stevens ${ }^{1}$;

Saul Greenberg'; Hartley Garfield ${ }^{2}$

${ }^{1}$ York University; ${ }^{2}$ University of Toronto, Toronto, Ontario

AIM: In community-based healthcare, health professionals often depend heavily on parents' abilities to discern and report infant symptoms of illness and well-being. Yet, research is very limited regarding what parental infant pain judgments are based on and how these judgments vary according to developmental stage. This paper sets out to better understand how parents make judgements about infant procedural pain.

METHODS: Parents and healthy infants were part of a community-based longitudinal cohort naturalistically observed during their 2-, 4-, 6-, and 12-month immunization appointments (OUCH Cohort; cross-sectional sample size range: $\mathrm{n}=408$ to 579 ) at 3 Toronto pediatrician clinics between 2007 and 2012.

RESULTS: Latent growth curve modeling determined that small to moderate amounts of variance in parent pain report was determined by infant pain behaviours $(\mathrm{R} 2=0.145$ to 0.331$)$. Noteworthy differences were found in the models for older versus younger infants, in relation to the roles of parental sensitivity, infant gender, parent age, and amount of variance explained by different infant pain variables.

CONCLUSIONS: Experts agree that infant behaviours are of the utmost importance in assessing infants in pain. However, current findings suggest parent pain judgments are not predominantly determined by infant pain behaviours, especially prior to 6 months of age. Researchers and clinicians must be cautious about the meaning of parent pain judgments for diagnostic and treatment purposes, especially if it differs from their own behaviourally-based pain assessment.

\section{P70}

CHARACTERISTICS OF PATIENTS SEEN IN PRIMARY CARE PRACTICE AS PART OF A PAIN PRECEPTORSHIP PROGRAM FOR FAMILY PHYSICIANS

Patricia Poulin ${ }^{1,2,3}$; Etienne Archambault ${ }^{3}$; Jonathan Yen ${ }^{1}$; Rebecca Small $^{1}$; Howard Nathan ${ }^{1,2,3}$; Catherine Smyth ${ }^{1,2,3}$ ${ }^{1}$ The Ottawa Hospital Department of Anesthesiology; ${ }^{2}$ The Ottawa Hospital Research Institute; ${ }^{3}$ The University of Ottawa, Ottawa, Ontario

AIM: Family physicians (FP) are often uncomfortable treating patients with chronic pain. Access to pain clinics is limited. The PainConnect program, an initiative of The Ottawa Hospital Pain Clinic, involved pain specialists going to FPs' clinics to assess patients and educate FPs about treatments. This study explores patients' characteristics.
METHODS: From January 2011 to March 2012 five pain specialists visited 122 FPs in the Champlain region. Patients completed a series of questionnaires including validated assessment instruments, such as the Brief Pain Inventory (BPI), the Patient Health Questionnaire-9, and the Opioid Risk Tool.

RESULTS: There were 330 completed questionnaires. Patients were predominantly women $(58.0 \%)$ with an average age of 53.9 years old $(\mathrm{SD}=15.5)$. Patients had on average been experiencing pain for 9.6 years $(\mathrm{SD}=8.6)$, reported severe pain $(\mathrm{M}=7.0, \mathrm{SD}=1.5)$, and significant pain interference $(\mathrm{M}=7.2, \mathrm{SD}=1.9)$. The majority of patients had previously been diagnosed with a mental health problem $(82.7 \%)$, and $76.2 \%$ had moderate to severe symptoms of depression. The majority $(71.0 \%)$ were currently prescribed opiates for their pain.

CONCLUSIONS: Patients assessed as part of PainConnect reported severe pain causing a high degree of disability, as well as mental health problems. The severity and complexity of the patients' presentation suggests that additional training and support for FPs is needed and that in some cases other models of care (e.g., shared care with inclusion of allied health) might be indicated. We are now piloting an interdisciplinary pain preceptorship program for FPs.

\section{P71 \\ TOOTH PULP STIMULATION DEPRESSES EXCITABILITY OF RAT FACE PRIMARY MOTOR CORTEX (FACE-MI) THAT IS DEPENDENT ON THE FUNCTIONAL INTEGRITY OF MEDULLARY ASTROCYTES}

Henry Pun; Laith Awamleh; Limor Avivi-Arber; Barry J Sessle University of Toronto, Toronto, Ontario

AIM: Dental pain is associated with neuronal and astrocytic processes in the medullary dorsal horn $(\mathrm{MDH})$ and neuroplastic changes in face-MI, and may be reflected clinically in disruption of orofacial sensorimotor functions. To examine if $\mathrm{MDH}$ astrocytes are involved in the latter, we tested whether application of the inflammatory irritant mustard oil (MO) to the rat's tooth pulp affects face-MI excitability defined by intracortical microstimulation (ICMS) and if MDH application of astrocytic inhibitor (Methionine Sulfoximine, MSO) influences this effect.

METHODS: Under Ketamine general anaesthesia, Sprague-Dawley male rats were implanted with electromyographic electrodes into the right and left anterior digastric (RAD, LAD) muscles. Following craniotomy, a microelectrode was positioned in the left face-MI $(<2.4 \mathrm{~mm}$ depth) at a site from which ICMS ( $35 \mathrm{~ms}$ train, $12 \times 0.2 \mathrm{~ms}$ pulses, $333 \mathrm{~Hz}, \leq 30 \mu \mathrm{A}$ ) could evoke $\mathrm{AD}$ responses. Baseline ICMS thresholds were monitored for 30 min, then $\mathrm{MO}(\mathrm{N}=6)$ (or saline $\mathrm{N}=5$ ) was applied to the previously exposed pulp and ICMS thresholds monitored for $60 \mathrm{~min}$. In other rats $(\mathrm{N}=5), 0.1 \mathrm{mM}$ MSO (or vehicle) was applied to MDH $15 \mathrm{~min}$ after MO application.

RESULTS: Within 15 min of MO pulp application, RAD ICMS thresholds significantly increased (34\%; repeated measures ANOVA, post-hoc Bonferroni: $\mathrm{p}=0.014$ ). Pulpal application of saline produced no significant changes in RAD thresholds. The decreased face MI excitability could be normalised to baseline levels following the MDH application of MSO but not following vehicle application.

CONCLUSIONS: These findings suggest that motor disturbances associated clinically with dental pain may involve reduced face-MI excitability that is dependent on the functional integrity of $\mathrm{MDH}$ astrocytes. Supported by CIHR MOP4918

\section{P72}

\section{PARENTAL VERBAL REASSURANCE DURING INFANT IMMUNIZATION: A DESCRIPTIVE EXAMINATION OVER THE FIRST YEAR OF LIFE.}

Nicole Racine'; Rebecca Pillai Riddell ${ }^{1,2}$ David Flora ${ }^{1}$; Hartley Garfield²; Saul Greenberg ${ }^{2}$

${ }^{1}$ York University; ${ }^{2}$ Hospital for Sick Children, University of Toronto, Toronto, Ontario

AIM: Understanding the frequency and occurrence of reassurance is important for management of infant pain-related distress. This study examined the occurrence of parental verbal reassurance across the first year of life during immunization. 
METHODS: A longitudinal sample of 154 parent-infant dyads from the OUCH at 2-, 4-, 6-, and 12 months of age were videotaped during immunizations. Parental verbal reassurance was measured one minute prior to the first immunization, as well as one and two minutes after the last needle.

RESULTS: A repeated measures ANOVA revealed that there was a significant main effect of infant age on parental use of verbal reassurance two minutes post-needle, $\mathrm{F}(3,459)=5.28, \mathrm{p}=0.001$. Pairwise comparisons revealed a significant difference between two $(\mathrm{M}=0.16, \mathrm{SD}=0.22)$ and four-months of age $(\mathrm{M}=0.11, \mathrm{SD}=0.17, \mathrm{p}=0.009)$ and between two- and six-months of age $(\mathrm{M}=0.11, \mathrm{SD}=0.17, \mathrm{p}=0.009)$, with parents using more verbal reassurance two minutes after the needle at two months of age. Two minutes post-needle, caregivers of two month-old infants used more verbal reassurance than caregivers of four- and six-month olds.

CONCLUSIONS: Higher levels of caregiver reassurance at 2-months may be related the infant's heightened response to pain at this age (Ipp et al., 2004). Additionally, 12-months of age has the most painful stimulus, potentially explaining the lack of difference in verbal reassurance use between two- and 12-months (Schechter et al., 2007). Caregivers use the most reassurance with infants who express the most distress.

\section{P73 \\ PHYSICIAN'S PERSPECTIVES ON THE OPIOID MANAGER(TM)}

Andrew Robertson ${ }^{1}$; Sander Hitzig ${ }^{1}$; Andrea D Furlan ${ }^{2}$

${ }^{1}$ University of Toronto; ${ }^{2}$ Toronto Rehabilitation Institute - UHN,

Division of Physiatry, University of Toronto, Institute for Work \&

Health, Toronto, Ontario

AIM: The Opioid Manager (OM) is a point-of-care paper tool for physicians, which summarizes Canadian clinical guidelines on the management of non-chronic cancer pain with opioids.(1) In order to evaluate the efficacy of the $\mathrm{OM}$, there is a need to better understand how physicians are using the OM, and how it is relevant to their practice.

METHODS: Semi-structured interviews (approx. 1 hour) were conducted on seven physicians in Ontario with expertise in pain management. One interview was excluded because the physician did not meet the inclusion criteria. Six interviews were analyzed using fundamental qualitative methodology. The technique of 'code-recode' was conducted by two analysts to verify content validity.

RESULTS: The following main themes emerged: 1) OM - communication tool; 2) OM - educational tool; 3) OM - clinical tool; 4) OM content/ design; 5) OM benefits; 6) Who OM is used with; 7) OM potential; and 8) Challenges of pain management. Physicians' commented the $\mathrm{OM}$ was a useful reference for helping their clinical decision-making regarding opioids, and used it to educate and communicate with their patients/colleagues. Although many felt the content/design of the OM had a number of good features, there was a need for modifications (i.e., merge with other tools, create electronic version). Given the challenges associated with pain management, a number of benefits were derived from using the OM (i.e., protection, building therapeutic alliance), and respondents' felt the tool had the potential to meet a number of unmet needs related to opioid management. CONCLUSIONS: Overall, the OM was viewed positively for improving pain management practices but further work is required to refine the tool's potential.

\section{REFERENCES:}

1. Furlan AD, Reardon R, Salach L. The opioid manager: a point-of-care tool to facilitate the use of the Canadian Opioid Guideline. J Opioid Manag 2012;8:57-61.

\section{P74}

EFFICACY OF LOW LEVEL LASER THERAPY (LLLT) IN LUMBAR DISCOPATHIC PAIN (RANDOMIZEDCONTROLLED STUDY)

Ali Samarbakhsh; Mitra Shojaeei; Meraj Hami Samarbakhsh Pt Clinic

AIM: The purpose of this study is evaluating the effect of LLLT for lumbar discopathic pain. We combined laser therapy with regular physiotherapy to compare the results.

METHODS: A group of 42 patients between 25 and 65 old applied to our out-patient clinics with complaints of lumbar discopathic pain that had diagnosed of discopathy with MRI. Patients randomized in 2 groups (21 patients in each group). Their pain was estimated objectively \& subjectively from 4 below tests before treatment, at the end of treatment (2 weeks, 5 days in each week without weekends) and 2 weeks after treatment.Group1: Laser therapy with physiotherapy \& Group2: physiotherapy without laser therapy. Tests: 1-Trigger point palpation pain. 2- VAS. 3 NHP: Nottingham health profile. 4- NPAINAD (non-verbal pain assessment in advanced dementia).

RESULTS: There was no significant difference between two groups before treatment. There was significant improvement in the active laser group with PT group at the end of treatment and 2 weeks after completion treatment $(\mathrm{p}<0.01)$. PT alone seemed to aid relieving pain significantly while LLLT with PT had superior significant effect in reducing pain of discopathy within 2 weeks follow up.

CONCLUSIONS: Therapeutic laser offer a safe often effective, easily utilized primary or adjunctive therapy that is relatively cost effective for clinician \& patient. LLLT is more effective in pain relief and in the improvement of functional ability \& quality of life thus LLLT can be an important adjunct especially in patients with adverse side effects to drugs and invasive treatment.

REFERENCES:

1. Euro Journal of Scientific Research.NO1. (2009).pp.76-81

2. Practical Pain Management (laser for chronic LBP. January/Feb 2007;38-41

3. Laser In Surgery \& Medicine 2005;33:339-43

4. Aral Hakguder,MD et al: efficacy of LLLT. Laser In Surgery \& Medicine. 2005;35:339-343229-235

5. Ali Gur MD et al: Efficacy of LLLTin management of chronic pain. Journal of American Medical Directors Association 2003;4:9-15

6. Mimmis Logdberg-Andersson et al .LLLT of myofacial pain. Journal of Clinical Nursing 2001;10:697-706

7. Wewers M.E \& Lowe N.K.A critical review of VAS. Laser therapy 1997;9:79-86

8. Labbe R. Skogerboe K, Davis H et al: Laser photo bio activation mechanisms. Laser in surgery and medicine. 1990;10:201-207

\section{P75}

\section{THE EFFECT OF AN INDIVIDUALIZED EDUCATION INTERVENTION VERSUS USUAL CARE ON PAIN FOLLOWING AMBULATORY INGUINAL HERNIA REPAIR}

Mona Sawhney ${ }^{1}$; Judy Watt-Watson²; Ellen Hodnett; Michael McGillion²; Sonia Sarkissian²

${ }^{1}$ North York General Hospital; ${ }^{2}$ Lawrence S Bloomberg, Faculty of Nursing, University of Toronto, Toronto, Ontario

AIM: Inguinal hernia repair (IHR) is a common ambulatory surgery procedure, after which many patients experience moderate to severe pain. This trial aimed to examine the effectiveness of an individualized pain education intervention for men having IHR.

METHODS: Participants $(\mathrm{N}=82)$ were randomized to either the intervention or usual care group pre-operatively. The intervention group received a pain education booklet about managing post-operative pain, and 2 support calls pre-operatively and after discharge home. The primary outcome was WORST 24 hour pain on movement on post-operative day 2. Secondary outcomes included pain at rest and movement, pain-related interference, pain quality, analgesics taken, and adverse effects at postoperative days 2 and 7 .

RESULTS: At day 2, the intervention group reported significantly lower scores for all pain intensity outcomes, including WORST $24 \mathrm{hr}$ pain on movement $(\mathrm{t}(\mathrm{df})=4.7(73), \mathrm{p}<0.001)$. Also on day 2 , pain-related interference scores for the intervention group were lower, but not significantly different with the bonferroini correction $(\mathrm{t}(\mathrm{df})=2.1(73), \mathrm{p}=0.04)$. Constipation was the adverse effect identified most often, by both groups, on days 2 and 7 . While no differences between groups were evident for pain intensity or related interference at day 7 , it was noteworthy that $36 \%$ of the total sample reported moderate to severe pain.

CONCLUSIONS: Evidence from this trial indicates that the intervention was effective in decreasing pain intensity and related interference at day 2. An adequately powered trial with a longer evaluation period is necessary to determine if this intervention is successful in decreasing the development of persistent post-surgical pain in patients undergoing IHR. 
P76

SPINAL AND PERIPHERAL ADENOSINE A1 RECEPTORS (A1RS) CONTRIBUTE TO ANTINOCICEPTION BY SYSTEMICALLY ADMINISTERED AMITRIPTYLINE, ACETAMINOPHEN AND TRAMADOL IN THE FORMALIN TEST

Jana Sawynok; Jean Liu; Allison Reid

Dalhousie University, Halifax, Nova Scotia

AIM: Activation of adenosine A1 receptors (A1Rs) in the spinal cord and at sensory nerve endings in the periphery leads to antinociception in several preclinical pain models. This study examined the contribution of such receptors to antinociception produced by systemic amitriptyline (AMI), acetaminophen (ACET) and tramadol (TRAM).

METHODS: Antinociception was assessed using the 2\% formalin test (phase 2, 12-60 mins) in normal mice (C57Bl6) and in mice lacking adenosine A1Rs due to gene deletion (+/+ and -/- verified by PCR).

RESULTS: In normal mice, AMI, ACET and TRAM, when administered systemically by intraperitoneal (i.p.) injection, all produce dose-related antinociception against phase 2 flinches resulting from injection of $2 \%$ formalin into the plantar hindpaw. Antinociception produced by AMI $3 \mathrm{mg} / \mathrm{kg}$, ACET $300 \mathrm{mg} / \mathrm{kg}$, and TRAM $35 \mathrm{mg} / \mathrm{kg}$, given i.p. 15 or $20 \mathrm{mins}$ before formalin, was inhibited by spinal (acute lumbar puncture) and by intraplantar (to ipsilateral hindpaw) DPCPX 10-15 nmol, a selective adenosine A1R antagonist. Injection of DPCPX into the contralateral hindpaw did not reverse antinociception, verifying a local peripheral action for i.pl. administration. Local delivery of the antagonist alone by both routes had no intrinsic effect on formalin responses. Reversal of the action of AMI and ACET by DPCPX was observed in mice that were A1R +/+, but not $-/-$, verifying the involvement of A1Rs in the reversal.

CONCLUSIONS: These results demonstrate that, in a model of ongoing pain, spinal and peripheral adenosine A1Rs contribute to the antinociception produced by systemic administration of three agents that are commonly used as analgesics in humans for various pain conditions. These interactions may contribute to the inhibition of all three agents by systemic caffeine, which blocks both A1Rs and A2ARs.

\section{P77}

PSYCHOMETRICS OF THE CESD-R IN PERSONS WITH CHRONIC PAIN

Geoffrey Schultz

Calgary Chronic Pain Centre, Alberta Health Services and Research Assistant Professor, Department of Clinical Neurosciences, Faculty of Medicine, University of Calgary, Calgary, Alberta

AIM: The revised version of the Centre for Epidemiologic Studies of Depression Scale (CESD-R) was published in 2004 by Eaton and colleagues to screen for depression as defined in the DSM-IV. To date, no study has documented the psychometrics of the test in persons with chronic pain though the test is used in chronic pain settings, and depression measurement is important. This study establishes the test performance using nonparametric item response theory (nIRT).

METHODS: 1151 individuals completed the CESD-R prior to program entry. Average age was 47 with $68 \%$ female. Average pain duration was 10.1 years and average pain level 6.2 on a 0 to 10 NRS. Analyses used Ramsays (1991) S algorithm coded with minor adaptation in R. The core routine, calculates the probability for option items for levels of depression. This is done by applying a gaussian kernel smoother to an option indicator matrix derived from the patient questionnaires. Additional analyses were computed in R based on c++ source code of Ramsays (2000) TestGraf application.

RESULTS: All items produced a monotonically increasing relationship between item ratings and depression severity, therefore were of acceptable quality. Item options varied in their ability to discriminate levels of depression. Plots for items of various quality levels are presented. Test information and reliability curves are presented as well as item means, standard deviations and biserial correlations with overall depression level.

CONCLUSIONS: This study finds that the CESD-R adequately measure depression in persons with chronic pain.
P78

A COMPARISON OF THREE CANADIAN PAIN MANAGEMENT PROGRAMS: TOWARD THE DEVELOPMENT OF OPEN AND SHARED STRUCTURES, PROCESSES AND OUTCOMES

Geoffrey Schultz ${ }^{1}$; Douglas Cane ${ }^{2}$; Ingrid Fedoroff ${ }^{3}$;

Alexander J Clark ${ }^{4,5}$

${ }^{1}$ Calgary Chronic Pain Centre, Alberta Health Services, Calgary,

Alberta; ${ }^{2}$ Pain Services, Capital Health, Halifax, Nova Scotia;

${ }^{3}$ Complex Pain Centre, St Pauls Hospital, Vancouver, British

Columbia; ${ }^{4}$ Professor of Anesthesia, Pain Management and

Perioperative Medicine, Dalhousie University; ${ }^{5}$ Medical Director,

Pain Services, Capital Health, Halifax, Nova Scotia

AIM: Pain management programs exist across Canada. The Canadian Pain Society and Canadian Pain Coalition have called for collaboration and a unified voice to advocate for the reduction of the burden of pain. Comparison of established programs could start this collaboration. Quality healthcare is a function of resource inputs (structure), service delivery models (process) and evaluation of results (outcome) - the Donabedian model. This framework is used to explore Canadian pain management programs (PMP) and to develop open and shared communication pathways.

METHODS: A convenience sample of three publicly funded PMPs was used: Capital Health Pain Self-Management Program, Halifax, St. Paul's Hospital Complex Pain Centre, Vancouver, and Calgary Chronic Pain Centre. Comparison points reflecting resource inputs (FTEs of healthcare disciplines), the service delivery models (medical/self-management/rehabilitation; individual/group care; open/time-limited), and results (effect sizes of pain reduction, mood improvement and reduction in disability) were established.

RESULTS: All three programs are described as self-management based but differ in the range and FTE of healthcare disciplines. All shared a group-based programming element and differed in the amount of individual-based care. Program durations ranged from a closed 4-week program with additional care, to a 12-week program to an open-ended program (attended for approximately 2 years). Effect sizes in pain reduction, mood improvement, and disability reduction were found to be $[0.32,0.45,0.28$ Halifax], [0.15, 0.69, 0.25 Vancouver], [0.81, 0.42, 0.52 Calgary].

CONCLUSIONS: Similarities and differences were found between programs. It is hoped that that this work serves as a call for further collaboration.

\section{P79}

\section{ASSESSING THE PREDICTIVE POWER AND CLINICAL USABILITY OF QUANTITATIVE SENSORY TESTING IN RESEARCH AND CLINICAL MILIEU}

Petra Schweinhardt ${ }^{1}$; M Catherine Bushnell ${ }^{2}$; G Lavigne ${ }^{3}$; P Rainville $^{3}$; S Marchand ${ }^{4}$; PL Jackson ${ }^{5}$; MA Ware ${ }^{6}$

${ }^{1}$ McGill University; ${ }^{2}$ National Center for Complementary and Alternative Medicine (NCCAM), National Institutes of Health; ${ }^{3}$ Universite de Montreal, Montreal; ${ }^{4}$ University of Sherbrooke, Sherbrooke; ${ }^{5}$ Laval University, Laval; ${ }^{6}$ McGill University Health Centre, Montreal, Quebec

AIM: In 2007, the Quebec Pain Research Network (QPRN) implemented the Quebec Pain Registry, a database of chronic pain patients described using demographic and clinical measures using standardized and validated tools. To expand the phenotypic measures, we developed a Quantitative Sensory Testing (QST) protocol to explore predictive power and clinical potential of a series of sensory tests.

METHODS: Testing was performed at seven research or clinical centers across Quebec. Twenty neuropathic pain patients and 60 were healthy volunteers were tested. In the healthy participants, sensitization was induced on one volar forearm using $0.075 \%$ capsaicin cream. The standardized testing battery compared the affected body site (patients) or the sensitized body site (healthy volunteers) to a control site and included tests for warm and cool detection thresholds, heat and cold pain thresholds, mechanical detection threshold, mechanical allodynia, temporal mechanical pain summation, vibration threshold and pressure pain threshold. RESULTS: The complete testing battery was completed in 35 minutes per body site ( $\pm 6.5 \mathrm{mins})$. 
Using within-subject control sites was superior to using across-subject control sites. Tests that best differentiated patients from healthy participants were pain summation and wind up, warm detection and heat pain threshold, pain pressure and mechanical detection. Principal component analysis showed that while participant condition was better assessed with warm detection and heat pain thresholds, other tests from the battery explained most individual variance.

CONCLUSIONS: The results are discussed in terms of ease of use and cost efficiency for practitioners.

\section{P80}

\section{MOVED TO P31A}

\section{P81}

\section{WORK RELATED INJURIES CAUSING OR AGGRAVATING} FIBROMYALGIA IN THE MEDICOLEGAL ARENA:

\section{A JURISPRUDENTIAL ANALYSIS}

Peter A Ste-Marie ${ }^{1}$; Mary-Ann Fitzcharles ${ }^{2}$; Yoram Shir ${ }^{2}$

${ }^{1}$ University of Montreal; ${ }^{2}$ McGill University Health Centre, Montreal, Quebec

AIM: Fibromyalgia (FM) may be alleged to occur following a workplace injury with request for compensation. Ontario workers who report soft tissue injuries leading to FM are compensated according to a chronic pain policy. We have examined decisions pertaining to FM by the Workplace Safety and Insurance Appeals Tribunal (WSIAT), the final level of appeal for rejected workers claims.

METHODS: Tribunal decisions relevant to FM, between June 2006 and December 2011, were examined by predetermined protocol.

RESULTS: The Tribunal accepted 10/15 (67\%) cases pleading aggravation of FM ( 5 manual, 3 clerical, 7 healthcare/education workers), 13 single injuries, 2 gradual, with site low back or neck in 13. The Tribunal accepted 73/123 (59\%) new onset FM (60 manual, 29 clerical, 30 healthcare/education workers, 4 unknown), $68 \%$ single events, $32 \%$ repetitive activity, with site low back for $44 \%$, shoulder/upper limb in $40 \%$. Time from injury to diagnosis of FM (available for 117) $4.3 \pm 4.1$ years, with $6.3 \pm 2.8$ physicians cited for each worker. Previous psychological illness, injuries, neck pain or back pain were present for $17 \%, 22 \%, 10 \%$, and $13 \%$ respectively, without statement of previous health for $26 \%$. The FM diagnosis was based on rheumatologist report in $74 \%$, and family physician in $13 \%$.

CONCLUSIONS: Over half of appeals for aggravation or causation of FM following a work-related soft tissue injury were upheld by the Tribunal. The attribution of causation of FM to mostly a single workplace traumatic event is contentious and requires further examination.

\section{P82}

\section{WEIGHTING OF EVIDENCE IN AN APPEALS TRIBUNAL} ADJUDICATING WORK RELATED INJURIES AS A CAUSATION OF FIBROMYALGIA

Peter A Ste-Marie ${ }^{1}$; Mary-Ann Fitzcharles ${ }^{2}$; Yoram Shir ${ }^{2}$ ${ }^{1}$ University of Montreal; ${ }^{2}$ McGill Unviersity Health Centre

AIM: Ontario workers denied compensation for a work-related injury causing fibromyalgia (FM), may appeal to the Workplace Safety and Insurance Appeals Tribunal (WSIAT). The adjudication process relies on available medical information, input from board "expert advisors" and a "medical discussion paper". We examined the Tribunal weighting of evidence in reaching a decision.

METHODS: Between June 2006 and December 2011, the WSIAT heard 123 appeals for new onset FM following a work-related soft tissue injury. RESULTS: The Tribunal cited the following for decisions on 123 claimants: previous health in $74 \%$, "discussion paper" in $40 \%$, reliability of testimony/medical report doubted for $12 \%$ claimants, $15 \%$ board experts, $23 \%$ specialists, $5 \%$ family physicians $(F P)$. Allowed $(n=73)$ vs. rejected $(n=50)$ appeals were not different for demographics, specific injury, time to diagnose FM, previous health, and numbers of MDs cited. Allowed vs. rejected appeals were weighted as follows: rheumatologist report $73 \%$ vs. $36 \%$, FP report $36 \%$ vs. $20 \%$, tender point count (TPC) $36 \%$ vs. $14 \%$, and temporality $23 \%$ vs. $30 \%$. For appeals allowed, the Tribunal was more likely to rely on rheumatologist report $73 \%$ vs. a FP report $36 \%(\mathrm{p}<0.0001)$.

CONCLUSIONS: Legal decisions are reliant on evidence brought forward; especially in causation of FM. Concerns regarding the process include neglect of prior health status, weight of a specialist diagnosis often after many years, TPC, and "medical discussion paper", with less importance attributed to the FP. Medico legal decisions regarding FM must be based on current knowledge and sound applicable evidence.

\section{P83}

\section{EVALUATION OF A NEW PAIN EDUCATION E-TOOL (PEET) BY CANADIAN EDUCATORS}

Pierre-Paul Tellier ${ }^{1}$; Emmanuelle Belanger ${ }^{2}$; Charo Rodriguez ${ }^{1}$; Nancy Posel ${ }^{3}$; Mark Ware ${ }^{1,4}$

${ }^{1}$ Department of Family Medicine; ${ }^{2}$ Department of Psychiatry; ${ }^{3}$ Molson Medical Informatics; ${ }^{4}$ Department of Anesthesia, McGill University, Montreal, Quebec

AIM: The Pain Education E-Tool (PEET) is an innovative use of e-learning methodologies to support undergraduate education, including pain assessment and management. This study describes pain educators' feedback upon viewing the preliminary version of the tool.

METHODS: A sample of pain educators from across Canada were asked to analyze PEET with a view to implementation in different medical curricula. We adopted a qualitative descriptive research design, and individual phone interviewing with participants as the main source of data. A thematic analysis technique was privileged for analyzing the data gathered. RESULTS: A total of 9 educators from across Canada with different professional backgrounds were interviewed. Several strengths and weaknesses of PEET were identified, including: a) cases model excellent communication and understanding of issues involved in pain management; b) PEET is a comprehensive resource for learning about pain, c) the tool displays relevant choices of cases. Overall PEET was seen as an innovative teaching tool. The weaknesses pinpointed included: a) PEET could be more succinct; b) references should be updated and expanded; c) need to address the context of the consultation; d) multidisciplinary aspects could be more emphasized. Technical limitations were identified. Several suggestions for improvement were offered, such as adding patient information resources. CONCLUSIONS: Educators viewed PEET as an innovative and comprehensive e-tool for medical learning in management of pain. They highlighted weaknesses which we plan to address in further versions. The next step includes changing platforms from Adobe Flash to Decision Simulation which will offer several advantages including formative evaluation of learners.

\section{P84}

ACCEPTANCE-BASED BEHAVIORAL THERAPY FOR FIBROMYALGIA: 1-YEAR FOLLOW-UP

Gregg Tkachuk ${ }^{1}$; Heather Simister²; Matthew Bailly'; Ryan Skrabek ${ }^{3}$; Barbara Shay ${ }^{4}$

${ }^{1}$ Department of Clinical Health Psychology, Faculty of Medicine; ${ }^{2}$ Department of Psychology; ${ }^{3}$ Section of Physical Medicine and Rehabilitation, Faculty of Medicine; ${ }^{4}$ School of Medical Rehabilitation, Faculty of Medicine, University of Manitoba, Winnipeg, Manitoba

AIM: Fibromyalgia syndrome (FMS) is a widespread chronic pain condition primarily affecting females. Acceptance-based therapies have shown promise in the treatment of chronic pain. This study presents the results of a pilot study investigating the effectiveness of an acceptance-based behavioural therapy (ABBT) for FMS.

METHODS: Twelve participants were recruited through a FMS support organization. ABBT consisted of 4 group sessions and 6 individual sessions over 3 months. Groups focused on psychoeducation and social support. Individual sessions involved experiential exercises promoting mindful acceptance and living a valued life. Baseline, post-treatment, 6-month and 1 -year follow-up measures evaluated FMS impact, mindfulness, pain acceptance, sleep, pain-related fear, depression, physical performance, values, and pain. Nine female participants completed treatment (Mean age: 44.9 years, Mean pain duration: 139 months). Due to the small sample, statistical significance was set at $\mathrm{p}<0.10$.

RESULTS: Results revealed significant improvements in pain $(\mathrm{p}=0.07)$, pain-related fear $(\mathrm{p}=0.01)$, and pain acceptance $(\mathrm{p}<0.01)$. Most patients reported an improved ability to live and function with FMS on global ratings. Six-month follow-up revealed maintained improvements in pain 
acceptance $(p<0.05)$ and pain-related fear $(p<0.01)$, and that general (mindful) acceptance increased $(\mathrm{p}<0.08)$. One-year follow-up revealed significant improvements in fibromyalgia impact $(\mathrm{p}=0.07)$, depression $(p<0.05)$, sleep latency $(p<0.01)$ and duration $(p<0.001)$, and maintained improvements in pain-related fear $(\mathrm{p}<0.05)$ and acceptance $(\mathrm{p}<0.05)$. CONCLUSIONS: The results are promising and suggest that a randomized controlled trial be conducted with a larger sample.

\section{P85}

\section{PROCESSES OF CHANGE IN CBT FOR IRRITABLE BOWEL SYNDROME}

\section{Gregg Tkachuk; Lesley Graff}

Department of Clinical Health Psychology, Faculty of Medicine, University of Manitoba, Winnipeg, Manitoba

AIM: Irritable bowel syndrome (IBS) is a functional bowel disorder with abdominal pain as a primary symptom. Cognitive-behavioural therapy (CBT) is an empirically supported treatment for IBS. However, there has been little evaluation of process variables that may mediate clinical outcomes of CBT. This study investigated relationships between several process variables and clinical outcomes, using effectiveness data. Process variables included motivation for change, bowel-specific cognitions, homework adherence, and coping skills self-efficacy. Clinical outcomes included pain and gastrointestinal symptom change, depression, anxiety, and quality of life (QOL).

METHODS: 80 IBS patients (mean age 37, 86\% female, 52\% married, $76 \%$ employed) completed validated self-report measures prior to and after attending a 10-session, protocol-based group CBT program, along with global ratings of symptom change and coping skills self-efficacy posttreatment.

RESULTS: $80 \%$ of patients reported improved IBS symptoms, with $67.5 \%$ reporting decreased pain post-treatment. Significant improvements were observed for depression $(\mathrm{p}<0.001)$, anxiety $(\mathrm{p}<0.001)$, and QOL $(\mathrm{p}<0.01)$. Changes in motivational variables and cognitions were significantly related to improved depression $(p<0.01 ; p<0.001)$, anxiety $(p<0.001$; $\mathrm{p}<0.01)$, and quality of life $(\mathrm{p}<0.05 ; \mathrm{p}<0.001)$. Homework adherence, selfefficacy, and motivational variables were associated with significantly improved symptom ratings $(\mathrm{p}<0.05 ; \mathrm{p}<0.05 ; \mathrm{p}<0.05)$ and QOL $(\mathrm{p}<0.05)$. Pre-treatment motivational variables predicted post-treatment coping selfefficacy $(p<0.05)$, improved pain and symptom ratings $(p<0.01)$, and physical health QOL $(\mathrm{p}<0.05)$.

CONCLUSIONS: Each of the process variables was significantly associated with some aspect of change in outcomes, suggesting these are important targets for treatment. Future research should examine the relative importance of these process variables and how they may interact to mediate outcomes.

\section{P86}

\section{MODELLING PAIN, DISABILITY, AND DEPRESSION IN A} MULTIDISCIPLINARY PAIN CENTRE

\section{Gregg Tkachuk; Matthew Bailly}

Department of Clinical Health Psychology, Faculty of Medicine, University of Manitoba, Winnipeg, Manitoba

AIM: To examine constructs hypothesized to have significant relationships with pain, disability, and depression to better understand the pain experience and identify treatment targets. Hypotheses: pain catastrophizing and pain-related fear would be positively associated, while pain acceptance and coping variables would be negatively associated, with these variables.

METHODS: Two hundred and forty-eight patients ( mean age $=45.06$, $\mathrm{SD}=11.26 ; 68 \%$ female) completed a battery of self-report questionnaires (McGill Pain Questionnaire (MPQ), Pain Disability Index (PDI), Beck Depression Inventory - II (BDI-II), Coping Strategies Questionnaire (CSQ), Chronic Pain Acceptance Questionnaire (CPAQ), Pain Catastrophizing Scale (PCS), and Tampa Scale for Kinesiophobia - 13 (TSK-13) routinely administered prior to receiving treatment. Pearson correlation coefficients were computed for all variables. All coefficients were in the hypothesized direction.

RESULTS: Twenty-four percent of the variance in MPQ scores was accounted for by a model containing significant coefficients for PSC Helplessness ( $p=0.001)$, TSK Somatic Focus $(\mathrm{p}=0.004)$, and CSQ Diverting Attention $(\mathrm{p}<0.001)$. Forty percent of the variance in PDI scores was explained by a model containing significant coefficients for PCS Rumination $(\mathrm{p}=0.01)$, MPQ Sensory $(\mathrm{p}<0.001)$, and CPAQ Activity Engagement $(p<0.001)$. Fifty-one percent of the variance in BDI scores was accounted for by a model containing Activity Engagement $(\mathrm{p}<0.001)$.

CONCLUSIONS: These results suggest that the pain constructs studied are important to assess and target for change during multidisciplinary pain treatment. Pain acceptance and catastrophizing appear to play a more significant role in the pain experience than the other variables studied.

\section{P87}

\section{PSYCHOMETRIC PROPERTIES OF THE COPING STRATEGIES QUESTIONNAIRE - REVISED}

Gregg Tkachuk' ${ }^{1}$; Cheryl Harris²; Matthew Bailly ${ }^{1}$

${ }^{1}$ Department of Clinical Health Psychology, Faculty of Medicine, University of Manitoba, Winnipeg, Manitoba; ${ }^{2}$ The Ottawa Hospital Cancer Centre, Ottawa, Ontario

AIM: The Coping Strategies Questionnaire (CSQ) is a 48 item, rationally derived scale composed of 8 factors. However, the 8 factor structure has been difficult to confirm statistically. Exploratory factor analysis found a 6 factor, 27 item version (Robinson et al, 1997). However, confirmatory factor analyses (CFA) of this revised CFQ (CFQ-R) have found only moderately acceptable fit (Riley et al, 1997) and lower than expected internal consistencies (Utne et al, 2009). The aims of this study were to determine whether the 6 factor structure of the CSQ-R could be replicated and to determine if the scales have adequate internal consistencies in a sample of patients attending a multidisciplinary pain clinic.

METHODS: Two hundred and seventy-seven patients (66\% female, mean age $=47.05, \mathrm{SD}=12.87)$ completed the CSQ as part of battery of selfreport questionnaires routinely administered prior to psychological treatment. Data were subjected to CFA using EQS software.

RESULTS: The 6 factor structure of the CSQ-R was confirmed with reasonable initial fit $(\mathrm{CFI}=0.92$ and RMSEA $=0.06)$. Post-hoc modifications including removal of 5 uncorrelated factor covariances and 4 correlated error terms yielded very good fit $(\mathrm{CFI}=0.95$ and RMSEA $=0.05)$. Internal consistency reliabilities for the individual scales were very good (Cronbach's alphas ranging from 0.80 to 0.87 ).

CONCLUSIONS: The CSQ-R is a psychometrically sound measure for use in multidisciplinary pain clinics. Further research is needed to determine the precise nature of the relationships among the individual factors and which factors may be meaningfully combined to represent higher order constructs.

REFERENCES:

1. Riley JL, Robinson, ME. CSQ: Five Factors or Fiction? Clin J Pain 1997;13:156-62.

2. Robinson ME, Riley JL, Myers CD, Sadler IJ, Kvaal SA, Geisser ME, Keefe FJ. The Coping Strategies Questionnaire: A Large Sample, Item Level Factor Analysis. Clin J Pain 1997;13:43-49.

3. Utne, I, Miaskowski C, Bjordal K, Cooper BA, Valeberg BT, Rustoen T. Confirmatory Factor Analysis of the Coping Strategies Questionnaire-Revised in Samples of Oncology Outpatients and Inpatients With Pain. Clin J Pain 2009;25:391-400.

\section{P88}

\section{PHARMACOKINETICS OF A NEW IMMEDIATE-RELEASE METHADONE TABLET FORMULATION WITH DECREASED IN VITRO SOLUBILITY}

\section{Robert K Vinson}

Paladin Labs

AIM: A new tablet formulation of methadone was developed with unique physicochemical properties, allowing for decreased solubility in aqueous solutions in in vitro studies. It was unknown whether this newly developed formulation would affect its bioavailability compared with the currently available formulation of methadone.

OBJECTIVE: The objective of this study was to further examine the in vitro solubility of the new methadone formulation, and to evaluate and compare the relative bioavailability and pharmacokinetics to the previous reference formulation after a single oral dose administration under fasting conditions.

METHODS: In vitro comparative dissolution for the new methadone formulation and the previous reference formulation was conducted. A 
randomized, single- dose, blinded, two-period, two-sequence, crossover pharmacokinetic study was performed in 24 healthy volunteers under fasting conditions.

RESULTS: The new methadone formulation demonstrated a $30-40 \%$ decrease in solubility over 30 minutes compared with the previous reference formulation at the highest dose; all other doses also showed decreased in vitro solubility. The pharmacokinetic parameters determined were: maximum plasma drug concentration (Cmax) of $38.1 \mathrm{ng} / \mathrm{mL}$; time to reach Cmax (tmax) of 2.80 hours; mean area under the plasma concentrationtime curve (AUC) from time zero to 72 hours (AUC72) of $1043 \mathrm{ng}-\mathrm{h} / \mathrm{mL}$; mean AUC from time zero to infinity (AUC $\infty$ ) of $1430 \mathrm{ng}-\mathrm{h} / \mathrm{mL}$; mean elimination rate constant (ke) of 0.0206 hours $(-1)$; and mean elimination half-life $\left(t^{1 / 2}\right)$ of 36.7 hours. These values are statistically equivalent to the previous reference formulation of methadone.

CONCLUSIONS: This study provides the first complete set of pharmacokinetic data on a novel methadone formulation. The formulation demonstrates decreased in vitro solubility while providing equivalent plasma methadone levels compared with the previous formulation.

\section{P89}

SURVEY OF CANADIAN INTERVENTIONAL PAIN

PHYSICIANS (CIPP): WHAT IS THE MINIMUM NUMBER OF PROCEDURES FOR COMPETENCY?

\section{Clint S Wong}

Pain Management Specialist

AIM: There is little literature in pain medicine regarding the minimum number of procedures required to become proficient in interventional techniques compared to anesthesia and other literature (1-5). Since the Royal College of Physicians and Surgeons recently recognized pain medicine as a subspecialty in Canada and as pain fellowships become accredited, the question of the "minimum number" of procedures arises. This survey of Canadian interventional pain physicians aims to answer: What is the minimum number of procedures a fellow must perform to reach competency?

METHODS: A 20-question voluntary online survey was emailed to the APDOC (Academic Pain Directors of Canada) Committee in February of 2012 after REB approval. Completion of the survey was regarded as implied consent. Responses were confidential. Statistical analysis using Excel and NCS 2007 (Statistical Software, 2007; Kaysville, Utah) was used to represent data.

RESULTS: Of 67 surveys sent, 21 responded (31.3\%). For common pain procedures, $42.9 \%(n=9)$ suggested 0 to 40 translaminar epidural procedures were required for competency; $33 \%(n=14)$ suggested 0 to 40 or 41 to 80 for transforaminal epidurals; $47.6 \%(n=10)$ suggested 0 to 40 for intraarticular facet injections; $38.1 \%(n=8)$ suggested 0 to 40 for medial branch nerve blocks; $47.6 \%(n=10)$ suggested 41 to 80 for medial branch rhizotomy; $76.2 \%(\mathrm{n}=16)$ suggested 0 to 40 for trigger point injections; and $47.6 \%(n=10)$ suggested 0 to 40 for pulsed radiofrequency interventions.

CONCLUSIONS: The preliminary survey results reveal insight on Canadian interventional pain physicians' views on procedural competency. Further investigation and re-surveying are needed to better capture prevailing views.

REFERENCES:

1. Stringer KR, Bajenov S, Yentis SM. Training in airway management. Anaesthesia 2002;57:967-83.

2. Sivaprakasam J, Purva M. CUSUM analysis to assess competence: what failure rate is acceptable? Clin Teach 2010;7:257-61.

3. Watterson LM, Hyde S, Bajenov S, Kennedy SE. The training environment of junior anaesthetic registrars learning epidural labour analgesia in Australian teaching hospitals. Anaesth Intensive Care 2007;35:38-45.

4. Naik VN, Devito I, Halpern SH. Cusum analysis is a useful tool to assess resident proficiency at insertion of labour epidurals. Can J Anaesth 2003;50:694-8.

5. Charuluxananan S, Kyokong O, Somboonviboon W, Pothimamaka S. Learning manual skills in spinal anesthesia and orotracheal intubation: is there any recommended number of cases for anesthesia residency training program? J Med Assoc Thai 2001;84 Suppl 1:S251-5.

\section{P90}

IMPROVING PAIN ASSESSMENT PRACTICES AND

OUTCOMES IN LONG-TERM CARE FACILITIES

Ramesh Zacharias ${ }^{1}$; Thomas Hadjistavropoulos ${ }^{2,3}$;

Sharon Kaasalainen ${ }^{4}$, Jaime Williams ${ }^{3}$

${ }^{1}$ Medical Director, Village of Erin Meadows; ${ }^{2}$ Department of

Psychology; ${ }^{3}$ Centre on Aging and Health, University of Regina,

Regina, Saskatchewan; ${ }^{4}$ School of Nursing, McMaster University,

Hamilton, Ontario

AIM: Prior research indicates that systematic, pain assessments by longterm care (LTC) staff improve pain management practices, but do not affect regularly-scheduled medication prescriptions. Our purpose was to determine whether a pain assessment protocol in LTC, involving regular communication with the residents' physicians, would encourage re-evaluation of prescriptions. This was deemed important because pain can produce behavioural disturbances, which clinicians could mistake as a manifestation of a psychiatric problem leading to psychotropic rather than analgesic medication prescriptions.

METHODS: One LTC facility implemented the intervention and a comparable facility served as control. A total of 67 residents participated. The intervention involved regular pain assessments by nursing staff, as well as weekly communication of elevated scores to resident physicians who also participated in monthly discussions about pain with a research team physician. Medications before and after the intervention were examined. Following the intervention, staff focus groups were conducted.

RESULTS: Analyses of Variance indicated that regularly-scheduled medication use decreased for intervention residents and increased for control residents. Follow-up analyses for specific medication classes showed significant intervention group reductions for regularly-scheduled benzodiazepines. Key qualitative themes related to the intervention increasing staff attention to pain, improving staff communication, and focusing efforts on understanding symptom etiology.

CONCLUSIONS: Due to the intervention, physicians re-evaluated their prescriptions, decreasing regularly-scheduled, benzodiazepines. This may have been due to better recognition of pain and is considered a positive finding because benzodiazepines can have serious side effects including elevated risk of injurious falls and disrupted cognition. Results have implications for problematic polypharamcy practices in LTC. 
A

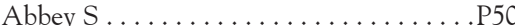

Ahmad SM ...................... 64

Ahmed S . . . . . . . . . . . . . . P38,P53

Ahola Kohut S.............22D,P22

Akhtar-Danesh N ............... 11A

Ali $\mathrm{H} \ldots \ldots \ldots \ldots \ldots \ldots \ldots \ldots$. . . . . . .

Alkokani M . ..................P39

Allen M . . . . . .................P59

Aly M....................... 20

Apkarian AV .................. 10A

Archambault E. ..................P70

Arratoon C. . . . . . . . . . . . . . . . P54

Asenjo JF . . . . . . . . . . . . . . . . . . P30

Asmundson GJG .............. P29A

Austin JS . . . . . . . . . . . . . . . . .P34

Avivi-Arber L . . . . . . . . . . . . P71

Awamleh L. . . . . . . . . . . . . . . . . . P71

\section{B}

Backman SB. . . . . . . . . . . . . . . P30

Baer PA . . . . . . . . . . . . . . $9 \mathrm{~B}$

Bailly M . . . . . . . . . . P40,P84,P86,P87

Becker S....................P14

Beggs S...................27A

Béland F. . . . . . . . . . . . . . . . .P43

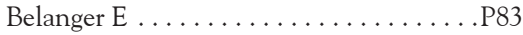

Bélanger $G \ldots \ldots \ldots \ldots \ldots \ldots \ldots$. . . . . . . . . .

Bellingham G............... 11G

Belzile-Lachapelle S. . . . . . . . . . . . . . .P43

Bennett GJ ....................10B

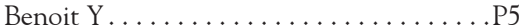

Bernier E . . . . . . . . . . P2,P31A,P32

Bhatnagar $\mathrm{S} \ldots \ldots \ldots \ldots \ldots \ldots \ldots . . . . . .64$

Birnie KA. . . . . . . . . . . . . . . . . . P3,P4

Boerner KE. . . . . . . . . . . . . . .P4

Boisjoli M-F. . . . . . . . . . . . . . . .P31

Boulay L.................... P42

Bourgault P.................... 43

Brazil K.................. 11A

Brecht K................... 15D

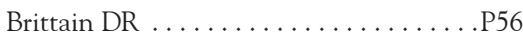

Brouse C........................ 62

Brown SC. . . . . . . . . . . . . . . P23

Bruins R....................P42

Brymer C ..................... 11G

Buckley DN ................ 11D

Buckley N. . . . . . . . . . . . . P45,P54

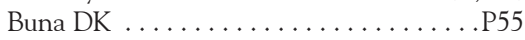

Bushnell MC .....................P79

\section{C}

Caes L. . . . . . . . . . . . 23,23A,P4,P5

Cahill C..............................

Campbell F. . . . . . . . . . . 11H,P23,P28

Campbell-Yeo M ................23B

Cane D. . . . . . . . . . . . P44,P47,P78

Cara C . . . . . . . . . . . . . . . . . P7

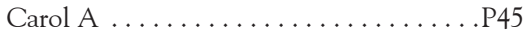

Ceko M .........................P46

Centofanti JE................ 11D

Chambers C....................P5

Chambers CT. . . . . . . . 2,2A,21,P3A,P4

Chan S . . . . . . . . . . . . . . . . . P6

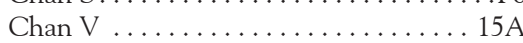

Chesworth B ..................11E

Choinière M .......... P49,P50,P52

Christou NV . . . . . . . . . . . . . . P30

Ciccone N . . . . . . . . . . . . . 15A

Clara I . . . . . . . . . . . . . . . 11C

Clark AJ . . . . . . . . . . . P47,P66,P78
Clarke H. . . . . . . . . . . . 3,27C,P21

Coleman R. . . . . . . . . . . . . . . . . P43

Cooper L .....................P45

Craig KD ........................

Cummings $\mathrm{G} \ldots \ldots \ldots \ldots \ldots \ldots \ldots \ldots \ldots$

Curtis $\mathrm{K} \ldots \ldots \ldots \ldots \ldots \ldots \ldots \ldots$. . . . . . .

\section{$\mathrm{D}$}

Daigle $K \ldots \ldots \ldots \ldots \ldots \ldots \ldots . . . \ldots 31$

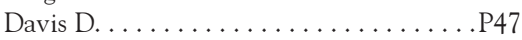

Davis KD .....................P36

Deshpande A...................P54

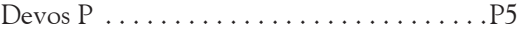

Deyell R . . . . . . . . . . . . ...11B

DiCenso A ................... 11A

Dittoe Barrett S . . . . . . . . . . . . P68

Dolovich L................. 11A

Donald F . . . . . . . . . . . . . . 11A

Dossett K . . . . . . . . . . . . . . . P34

Ducharme J ................17,17A

Duncan R. . . . . . . . . . . . . . . . .P60

Dupuis F....................

Dworkin RH ............... 1A

\section{$\mathrm{E}$}

Easson A .................... 15A

Ennis JH. .................... 37

Escobar MR . . . . . . . . . . . . . . P27

Esplen MJ...................P17

Estabrooks CA................ 11H

F

Fareg N...................... P61

Faregh N. . . . . . . . . . . . . . . .P48

Fashler SR . . . ......P9,P10,P27,P28

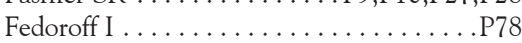

Finlayson R .................P38

Finlayson RJ . . . . . . . . . . . . . . P61

Fitzcharles M-A . . . . . . 18,P46,P48,P49,P50,

$\ldots \ldots \ldots \ldots \ldots \ldots \ldots$ P52,P81,P82

Flannery JF. . . . . . . . . . . . . P51,P59

Flora D . . . . . . . . . . . P22,P69,P72

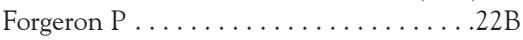

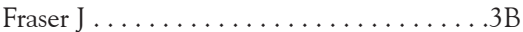

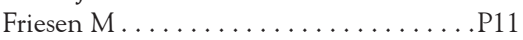

Frizzell B. . . . . . . . . . . . . . 15F

Furlan AD ............ P51,P59,P73

Fuss $S \ldots \ldots \ldots \ldots \ldots \ldots$ P12,P29A

\section{G}

Gagliese L. . . . . . . . . 19,15A,19C,P33

Gagnon C . . . . . . . . . . . . . . . P68

Gagnon M ...................P18

Gagnon MM ..................P13

Galonski M ...................P29

Gandhi W ....................P14

Garfield H . . . . . . . . . . . . . . . . P69,P72

Garland R. . . . . . . . . . . . . . P31A

Gaumond I. . . . . . . . . . . . . . . . . .P16

Gauthier J................... 31

Gauthier L . . . . . . . . . . . . . . . . . . 19B

Gauthier LR. . . . . . . . . . . . . . P33

Gélinas C......................P43

Ghandehari OO.................P15

Giladi H. . . . . . . . . . . . . . . 15D,P52

Gilani A..................... 66

Gilbert J ................. 11D

Gilberto A ...................... P57

Goffaux P .............. P16,P31

Gogovor A .............. P38,P53
Goldenberg DL . ............ P49,P50

Goldstine I. . . . . . . . . . . . . . . . .P54

Gomez C . . . . . . . . ......... 11D

Gordon A. ................ P29,P66

Görges M ................ 11B,P62

Gosselin É . . . . . . . . . . . . . . . . . .P43

Goubert L. . . . . . . . . . . . . . . . . . . . . P5

Gougeon V...................P16

Graff L . . . . . . . . . . . . . . . . . . P85

Greenberg S. . . . . . . . . . P69,P72

Grieve K. . . . . . . . . . . . . ....P55

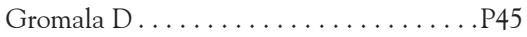

Guo S-L . . . . . . . . . . . . . . . . . P17

Gyurcsik NC ............. 7B,P56

\section{$\mathrm{H}$}

Hadjistavropoulos T. . . 11A,24,24B,P6,P13, ...... P15,P18,P90

Hami M . . . . . . . . . . . . . . . . . P74

Hamilton C . . . . . . . . . . . . . . . .11E

Hampton A ....................... 18

Hampton AJD ....................P15

Hansen N. . . . . . . . . . . . . . . . 11C

Harman K. ................. 11F,4C

Harris C . . . . . . . . . . . . . . . . .P87

Harrison JM . . . . . . . . . . . . . . . .P19

Harrison MB ....................P24

Hathaway G................ 15C

Hébert T. . . . . . . . . . . . . . . 15D

Hellsten L. . . . . . . . . . . . . . . . . . . P60

Henry J. . . . . . . . . . . . . . . . . .26B

Henry JL. . . . . . . . . . . . . . . . . 22,22C,P23

Hildebrandt D . . . . . . . . . . . . 15B

Hitzig S. . . . . . . . . . . . . . . . . . . P73

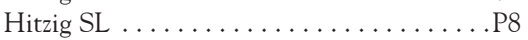

Hodnett E . . . . . . . . . . . . . . . . .P75

Holens PL . . . . . . . . . . . . . . . . . . P57

Honey CR . . . . . . . . . . . . . . . . . P20

Hooper RA. . . . . . . . . . . . . . . 15F

Howell D . . . . . . . . . . . . . . . P17

Hunter J . . . . . . . . . . . . . . . . 8A

Hutzul G................. 22A

\section{I}

Inman $R \ldots \ldots \ldots \ldots \ldots \ldots \ldots$. . . . . . . . . Isaac $\mathrm{L} \ldots \ldots \ldots \ldots \ldots \ldots \ldots \ldots$. . . . . . . . 23, P28

Isaacs AM. . . . . . . . . . . . . . . . P20

Isbester K . . . . . . . . . . . . . . 15C

Jabusch K.....................P58

Jackson PL .......................

Jibb L .................... 12C

Joseph L . . . . . . . . . . . . . . . 15D

Joshi S ......................... 64

Jull G ................... 15F

\section{$\mathrm{K}$}

Kaasalainen S. . . . . . . . . . . . . 11A,P90

Kam A ...................P59

Katz J . . . . . . 2B,21A,27,27B,P8,P10,P11,

$\ldots \ldots \ldots \ldots \ldots$ P12,P21,P27,P28,P29A

Khadra C . . . . . . . . . . . . . . . . P7

$\operatorname{Kim} \mathrm{G} \ldots \ldots \ldots \ldots \ldots \ldots \ldots \ldots \ldots \ldots$

Kinzel A........................P60

Klassen K . . . . . . . . . . . . . . . . P57

Kleiman V . . . . . . . . . . P P10,P21,P27

Ko G................. P49,P50

Koren G.............. 15A,15B 
$\mathrm{L}$

Lachance Fiola J. . . . . . . . . . . . P7

Lalloo C . . . . . . . . . . . . . . . 12B,P23

Lamb L. . . . . . . . . . . . . . . . . . . . .P61

Lamontagne $\mathrm{F} \ldots \ldots \ldots \ldots \ldots \ldots \ldots . . . . . \ldots 43$

Latimer $\mathrm{M} \ldots \ldots \ldots \ldots \ldots \ldots, 4 \mathrm{~A}$

Lauder $\mathrm{G} \ldots \ldots \ldots \ldots \ldots \ldots$ 11B,P62

Lauwers A ...................... 15B

Lavigne G. . . . . . . . . . . . . . . P79

LeFort S . . . . . . . . . . . . . . . . P24

Leger $P \ldots \ldots \ldots \ldots \ldots \ldots \ldots \ldots \ldots$. . . . . . . .

Le May $S \ldots \ldots \ldots \ldots \ldots \ldots \ldots \ldots$.

Lemay $S \ldots \ldots \ldots \ldots \ldots \ldots \ldots \ldots \ldots \ldots$

Léonard G . .................P31

Leong $\mathrm{N} \ldots \ldots \ldots \ldots \ldots \ldots \ldots \ldots \ldots$. . . . . . .

Levitin D . . . . . . . . . . . . . . 15C

Lewthwaite B................. P58

Li M .................... 15A

Librach SL . . . . . . . . . . . . . . . . . P33

Liu J .......................P76

Lofland K......................P68

Loh E .................... 11G

Lynch M. . . . . . . . . . . . . 5 5,P3,P66

Lynch ME. . . . . . . . . . . . . . 8,14,14B

\section{$\mathbf{M}$}

MacDonald R................ 3C

MacDougall P.................. 3A

Madadi $P \ldots \ldots \ldots \ldots \ldots \ldots \ldots \ldots \ldots$

Mailis-Gagnon A.................P39

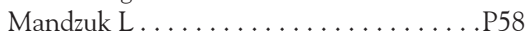

Mann E . . . . . . . . . . . . . . . . . .P24

Marchand S . . . . . . . . . . . . 9A,P16,P79

Marshall D ................. 11A

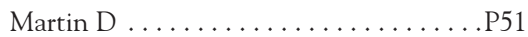

Martin L................... 15C

Martin-Misener R . . . . . . . . . . 11A

Matusa E. .....................P45

McAiney C . . . . . . . .......... 11A

McCarthy G................ 11G

McCarthy M . . . . . . . . . . . . . . . . P44

McDougall JJ ...................9

McEwan R ..................5B

McFetridge-Durdle J . . . . . .........P26

McGillion M . . . . . . . . 18A,21B,P26,P75

McGinnis E . . . . . . . . . . . . . . . . P45

Menec V.................. 11C

Mercier G. . . . . . . . . . . . . . . .P7

Mirali S . ................. 15C

Mishra S. .......................P64

Mogil J .................. 15C,5A,P34

Mogil JS .................. 1,2C

Montbriand J . . . . . . . . . . . . . . P65

Montbriand M . . . . . . . . . . . . . . .P65

Montgomery L . . . . . . . . . . . . . . 6

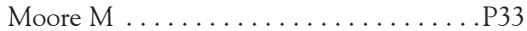

Moore $S \ldots \ldots \ldots \ldots \ldots \ldots \ldots \ldots$. . . . . . . .

Morley-Forster PK . . . . . . . . . . . .11G,P66

Moulin D . .....................20

Moulin DE ......... 11G,P49,P50,P66

\section{$\mathbf{N}$}

Nathan H. . . . . . . . . . . . . . . P66,P70

Nelson C . . . . . . . . . . . . . . 25C

Niederstrasser N. . . . . . . . . . . . 15C

Niederstrasser NG . . . . . . . . . . P25,P32

Noel M.................. P29A

\section{$\mathrm{O}$}

O'Keefe-McCarthy S.............P26

Oster H. . . . . . . . . . . . . . . . . . . . . P22

\section{$P$}

Pagé $G \ldots \ldots \ldots \ldots \ldots \ldots \ldots \ldots$. . P12

Pagé GM .................. P29A

Pagé MG ............ P10,P27,P28

Paneduro D . . . . . . . . . . . . . . . . . P29

Panopalis P. . . . . . . . . . . . . . . . P49, P50

Parker JA .................... P3A

Parkerson HA ............... P29A

Peng P .................... 14A

Pereira JX . . . . . . . . . . . . . . . 18C,P49,P50

Perlman R .................. 15D

Pfammatter AF. . . . . . . . . . . . . P68

Pillai Riddell R. . . . . . . . . . . P22,P69,P72

Poon E ..................... 11D

Posel N. . . . . . . . . . . . . . . . . . .P83

Potvin S..........................

Poulin P ........................ P70

Price R . . . . . . . . . . . . . . . P14,P30

Prkachin KM ................ 24A

Proulx J ................. P49,P50

Pun $\mathrm{H} \ldots \ldots \ldots \ldots \ldots \ldots \ldots \ldots \ldots \ldots \ldots$

\section{$\mathbf{R}$}

Racine $\mathrm{N} \ldots \ldots \ldots \ldots \ldots \ldots \ldots \ldots \ldots$. . . . . . . .

Rainville P . . . . . . . . . . . . . . . P79

Raju S. . . . . . . . . . . . . . . . . . .P29

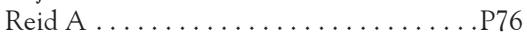

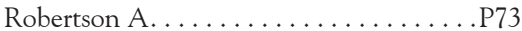

Robeson P .................... P54

Rodin G.................15A,P33

Rodriguez C . . . . . . . . . . . . . . . . P83

Rojas-Fernandez C. . . . . . . . . . . . .P35

Roosen KM ................ P10,P27

Roy $\mathrm{S} \ldots \ldots \ldots \ldots \ldots \ldots \ldots \ldots . . . . \ldots 31$

\section{$\mathrm{S}$}

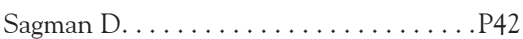

Samarbakhsh A ................... P74

Sapolsky R . . . . . . . . . . . . . . . 15C

Sarkissian S . . . . . . . . . . . . . P75

Saunders N. . . . . . . . . . . . . . . . . . .P8

Sawhney M ..................... P75

Sawynok J . . . . . . . . . . . . . . . 14C,P76

Schacht A ..................... 442

Schinkel M .....................P4

Schneider G.................15F

Schnell-Hoehn K. . . . . . . . . . . . . . . . . P58

Schultz G .............. P77,P78

Schweinhardt P . . . . . . 13,13A,P14,P30,P79

Scott SD ................ 11H

Scott W ........ 15E,25B,P1,P2,P31A,P32

Sekhon K....................P9

Sessle BJ . . . . . . . . . . . . . . . . . .P71

Shapiro M ................ 20A

Shay B . . . . . . . . . . . . . . . . . . P84

Shepherd FA . . . . . . . . . . . . P33

Shir Y....... 15D,P49,P50,P52,P81,P82

Shojaeei M...................P74

Shooshtari S. . . . . . . . . . . . . . 11C

Simister H . . . . . . . . . . . . P57,P84

Skrabek R. . . . . . . . . . . . . . . . . . .P84

Slayter K. . . . . . . . . . . . . . . 20A

Slepian P ................ 15C,P32

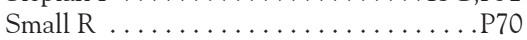

Smith A ..................... 15F

Smith AJ ....................P29

Smith M.................. 11B,P62

Smyth C. ................. P66,P70

Ste-Marie PA . . . . . . . . 18B,P46,P48,P49,

$\ldots \ldots \ldots \ldots \ldots \ldots \ldots$ P50,P81,P82

Sterling M .................... 15F

Sternberg W............... 15C

Stevens B . . . . . . . . . . . . . 11H
Stevens $\mathrm{S} \ldots \ldots \ldots \ldots \ldots \ldots \ldots \ldots$. . . . . . . . . . . . 69

Stinson J. . . . . . . . . 11H,19A,24C,P28

Stinson JN . . . . . . . . . . . . . . . . . P23

Stone L.................... 16A,P30

Stone LS ...................10

Sukosd M...................P34

Sullivan M........ 23C,P25,P31A,P32

Sullivan MJL ........ . 15E,25A,P19,P1,P2

Sumpton J . . . . . ..............P45

Sung Y-C ......................

Symeonidis I. ..................P53

\section{$\mathrm{T}$}

Tellier P-P ....................P83

Timmons BW................ 7C

Tkachuk G. . . . . . . P P40,P84,P85,P86,P87

Toth C ...................P66

Tousignant-Laflamme Y. . . . . . . . . . . .P43

Tran KT .......................... 33

Trang T $\ldots \ldots \ldots \ldots \ldots \ldots \ldots, 6,6$ A

Tremblay I . . . . . . . . . . . . . .

Trost Z . . . . . . . . . . . 15C,P19,P2

Tupper SM . . . . . . . . 7,7A,12,12A,16C

Tuttle A ...................P34

\section{$\mathrm{U}$}

Unruh A

\section{$\mathrm{V}$}

Vachon M-F..................P7

VanDenKerkhof E . . . . . . . . . . P24,P66

Verlooy J......................P5

Vernon H. . . . . . . . . . . . . . 26,26A

Versloot J ....................P9

Vervoort T .....................P5

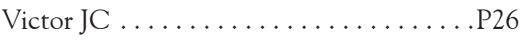

Vinson RK . . . . . . . . . . . . . . . .P88

Visca R................. P38,P53

Vlaeyen J ....................P25

\section{W}

Walker S. ....................P62

Walton A . . . . . . . . . . . . . . . . . . P11

Walton D . . . . . . . . . . . . . . .

Ware M .......... 15D,P38,P52,P83

Ware MA ..........16,16B,P66,P79

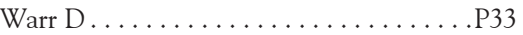

Watt-Watson J . . . . . . . . . . . . . P17,P75

Weber H. ...................... 35

West N.................. . 11B,P62

Wheeler B . . . . . . . . . . . . . . . P58

Wicks C . . . . . . . . . . . . . .P8

Wideman T . . . . . . . . . . . . . P32

Williams J..........P13,P15,P18,P90

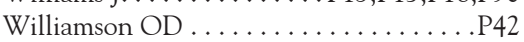

Winkelstein B ............... 26C

Winton P . . . . . . . . . . . . . . .11B

Wong CS ........................ 89

Wong $S \ldots \ldots \ldots \ldots \ldots \ldots \ldots \ldots \ldots$

Wu Q ........................

$\mathrm{Y}$

Yachnin D ..................... P34

Yamada J. . . . . . . . . . . . . . . . . . 11H

Yen J........................ . . . . .

Yin XC ........................... 37

$\mathrm{Z}$

Zacharias R. ............... P35,P90

Zawaly K..................11C

Zimmermann C.................P33

Zoheiry N. . . . . . . . . . . . . . . . . . . . P39 


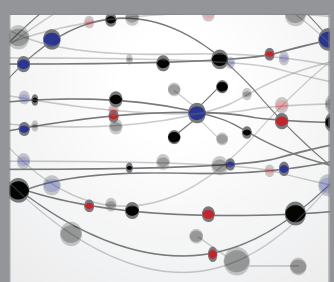

The Scientific World Journal
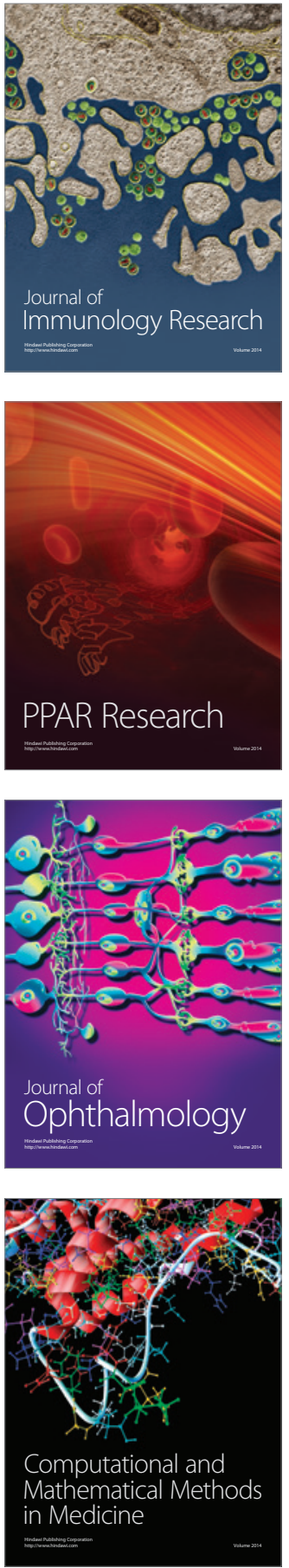

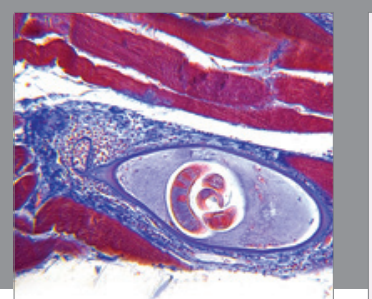

Gastroenterology Research and Practice

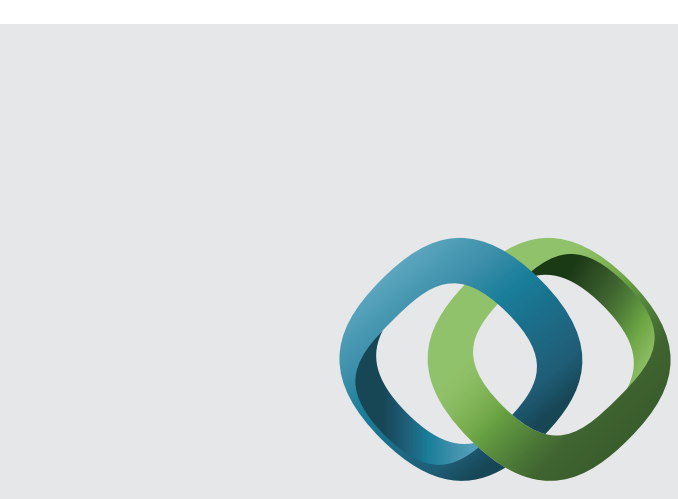

\section{Hindawi}

Submit your manuscripts at

http://www.hindawi.com
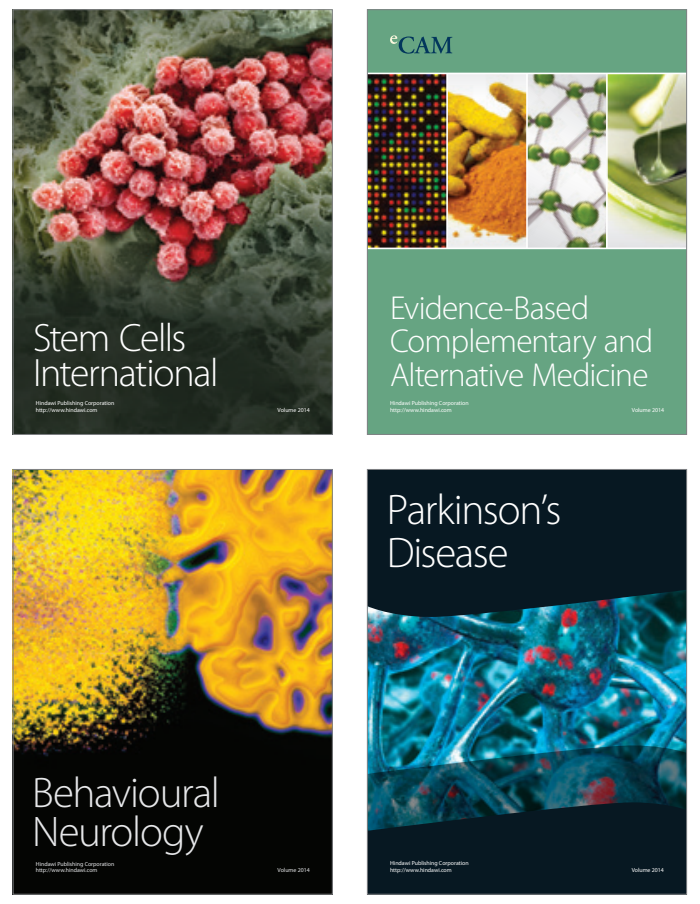
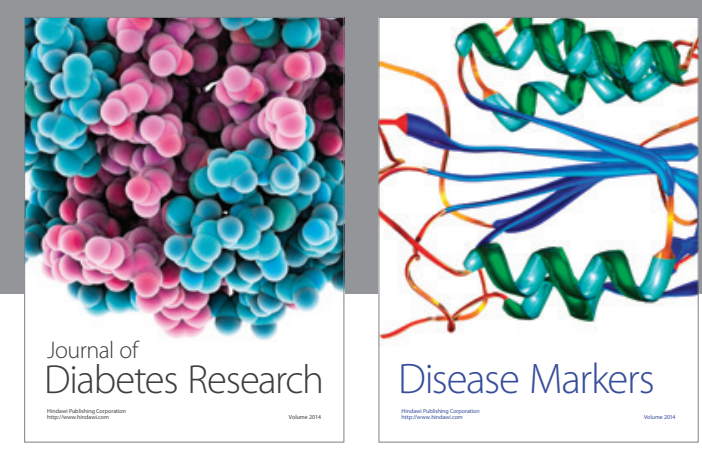

Disease Markers
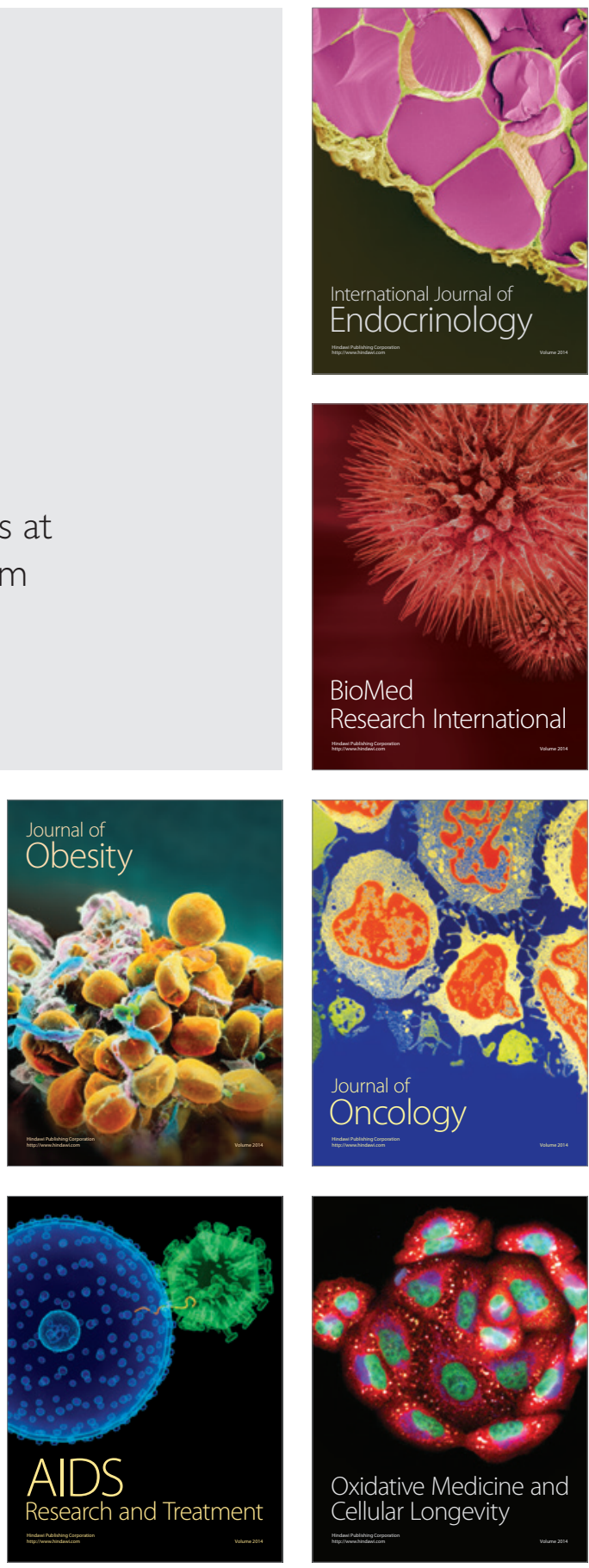